\title{
Contact Isotropic Realisations of Jacobi Manifolds via Spencer Operators
}

\author{
María Amelia SALAZAR ${ }^{\dagger}$ and Daniele SEPE ${ }^{\ddagger}$ \\ † IMPA, Estrada Dona Castorina 110, Rio de Janeiro, Brazil 22460-320 \\ E-mail: mariasalazarp@gmail.com \\ URL: http://w3.impa.br/ salazarp/ \\ $¥$ Universidade Federal Fluminense, Instituto de Matemática, Departamento de Matemática \\ Aplicada, Rua Mário Santos Braga S/N, Campus do Valonguinho, Niterói, Brazil 24020-140 \\ E-mail: danielesepe@id.uff.br \\ URL: https://sites.google.com/site/danielesepe/
}

Received October 07, 2016, in final form May 17, 2017; Published online May 25, 2017 https://doi.org/10.3842/SIGMA.2017.033

\begin{abstract}
Motivated by the importance of symplectic isotropic realisations in the study of Poisson manifolds, this paper investigates the local and global theory of contact isotropic realisations of Jacobi manifolds, which are those of minimal dimension. These arise naturally when considering multiplicity-free actions in contact geometry, as shown in this paper. The main results concern a classification of these realisations up to a suitable notion of isomorphism, as well as establishing a relation between the existence of symplectic and contact isotropic realisations for Poisson manifolds. The main tool is the classical Spencer operator which is related to Jacobi structures via their associated Lie algebroid, which allows to generalise previous results as well as providing more conceptual proofs for existing ones.
\end{abstract}

Key words: Jacobi structures; contact manifolds; Poisson structures; projective structures; contact actions

2010 Mathematics Subject Classification: 53D10; 53D17; 53D20; 37J15

\section{Contents}

1 Introduction $\quad 2$

2 Basic notions and motivation $\quad 4$

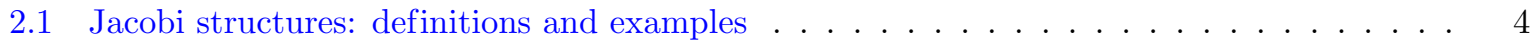

2.2 The Lie algebroid and Spencer operator of a Jacobi manifold . . . . . . . . . . . . . 6

2.3 Motivating example: Multiplicity-free actions on contact manifolds . . . . . . . . . . 8

3 Contact isotropic realisations of Jacobi manifolds $\quad 11$

4 Classification of contact isotropic realisations $\quad 14$

4.1 The period lattice and the Chern class . . . . . . . . . . . . . . . . . . . 14

4.2 Period lattices as transversal $\mathbb{Z}$-projective structures $\ldots \ldots \ldots \ldots$

4.3 The realisation problem for CIRs $\ldots \ldots \ldots \ldots \ldots$

5 The case of Poisson manifolds: comparing symplectic and contact isotropic realisations $\quad \mathbf{2 2}$

5.1 Symplectic isotropic realisations of Poisson manifolds: a reminder . . . . . . . . . . . . 22

5.2 Contact isotropic realisations of Poisson manifolds . . . . . . . . . . . . . . . 25

5.3 Strong transversal $\mathbb{Z}$-affine structures are $\mathbb{Z}$-projective $\ldots \ldots \ldots \ldots$

This paper is a contribution to the Special Issue "Gone Fishing". The full collection is available at http://www.emis.de/journals/SIGMA/gone-fishing2016.html 
5.4 Symplectic vs contact isotropic realisations of Poisson manifolds $\ldots \ldots \ldots$. . . . . . . 30

A Properties of regular Jacobi manifolds all of whose leaves are even dimensional $\quad 33$

B Proofs of the main results of Section 2.3 $\quad 35$

$\begin{array}{lll}\text { C } & \text { Missing proofs from Sections } 4.2 \text { and } 4.3 & 38\end{array}$

References

\section{Introduction}

Poisson structures can be considered as infinitesimal objects by thinking of them as Lie algebroid structures on cotangent bundles together with the canonical symplectic form. One approach to understand the geometry of a Poisson manifold is to consider its 'representations', which correspond to symplectic realisations (cf. [4,31]). The representations of smallest dimension, which are known as isotropic, play an important role, for they enjoy special geometric properties, such as the existence of a $\mathbb{Z}$-affine structure transverse to the symplectic foliation (cf. [6, 11]). Moreover, they are related to multiplicity-free actions of compact Lie groups on symplectic manifolds (cf. [11, 19]), as well as to the recently introduced notion of 'compactness' in Poisson geometry (cf. $[5,6]$ ). However, regarding Poisson manifolds as being Jacobi, the associated Lie algebroid structure is defined on the first jet bundle of the manifold, which is endowed with a canonical contact form. This point of view sheds a different light on Poisson geometry. For instance, in this case, the 'representations' are contact realisations. Continuing with the above analogy, a natural question is to study geometric properties of those representations of smallest dimension and to investigate whether they are connected with Hamiltonian Lie group actions in contact geometry as well as a slightly more general notion of 'compactness' for Poisson and Jacobi manifolds. The aim of the present paper is to begin to address the above question, with a view to continue this line of research in future papers.

A Jacobi structure on a manifold $M$ is a real line bundle $L \rightarrow M$ whose space of sections $\Gamma(L)$ is endowed with a local Lie bracket $\{\cdot, \cdot\}$ (cf. Definition 2.1); these generalise Poisson structures and contact manifolds (cf. Section 2.1). A Jacobi manifold $(P, L,\{\cdot, \cdot\})$ is encoded in a Lie algebroid structure on the first jet bundle $J^{1} L$ which is compatible with the classical Spencer operator (cf. Note 2.17). Contact realisations of $(P, L,\{\cdot, \cdot\})$ are representations of the Lie algebroid $J^{1} L$ : these are surjective submersions $\phi:(M, H) \rightarrow(P, L,\{\cdot, \cdot\})$, where $(M, H)$ is a contact manifold and $\phi$ is a Jacobi morphism satisfying a transversality condition (cf. Definition 3.1). The main object of study of this paper are contact isotropic realisations, which can be thought of as being of smallest dimension (cf. Definition 3.2 and Note 3.3).

As motivation, it is shown that these objects arise naturally when considering contact manifolds and their 'integrable' symmetries; some of the results may be of independent interest. For instance, contact isotropic realisations arise when considering the contact analogue of multiplicity-free Hamiltonian actions on symplectic manifolds (cf. Definition 2.21); these are the non-abelian analogues of contact toric manifolds studied in [25, 32]. The main results of this paper concern the classification of contact isotropic realisations to a suitable notion of isomorphism. These results are analogous to those of [11] for symplectic isotropic realisations of Poisson manifolds and generalise $[1,21]$. In particular,

- given a contact isotropic realisation of a Jacobi manifold, we show the existence of a $\mathbb{Z}$ projective structure transversal to the foliation on the Jacobi manifold (cf. Section 4.2 and Appendix C); 
- we provide cohomological criteria to determine whether there exists a contact isotropic realisation inducing a given transversal $\mathbb{Z}$-projective structure and to classify, up to isomorphism, all such realisations (cf. Theorem 4.18).

The methods employed in this paper are completely different from those of $[1,11,21,25,32]$, for the main tool used here is the classical Spencer operator and its properties (developed in greater generality in [8]), which allows to deal with Jacobi structures defined on non-trivial line bundles as well as providing a more conceptual approach to the case of Jacobi brackets on trivial line bundles. For instance, en route to proving Theorem 4.18, a local contact classification of contact isotropic realisations is attained, providing a different, more intrinsic proof to [21, Theorem 4$]^{1}$.

Lastly, we go back to Poisson manifolds, for which there is a natural question: what relation is there between existence of a contact isotropic realisation and that of a symplectic isotropic realisation? The methods developed in this paper allow to tackle the above question in the case in which a Poisson manifold admits transversal $\mathbb{Z}$-affine and $\mathbb{Z}$-projective structures which are 'related'. First, it is shown that a transversal $\mathbb{Z}$-affine structure satisfying an 'integrality' condition (cf. Definition 5.13) induces naturally a transversal $\mathbb{Z}$-projective structure (cf. Corollary 5.14). Intuitively speaking, this relation should be viewed as analogous to the following construction: given an integral symplectic manifold, it is possible to construct a Boothby-Wang-type contact manifold (cf. Example 3.4). In fact, the last main result of this paper generalises the above construction: given transversal $\mathbb{Z}$-affine and $\mathbb{Z}$-projective structures (denoted by $\Xi$ and $\Sigma$ respectively) on a Poisson manifold $(P, \Lambda)$ related as above, there exists a contact isotropic realisation of $(P, \Lambda)$ inducing $\Sigma$ if and only if there exists a symplectic isotropic realisation of $(P, \Lambda)$ inducing $\Xi$ whose total space has an integral symplectic form (cf. Theorem 5.20).

The structure of the paper is as follows. Section 2 introduces the basic notions used throughout the paper, sets the notation and provides motivating families of examples to study contact isotropic realisations. Seeing as the approach to contact and Jacobi manifolds taken in this paper follows [7] and differs from the standard one taken in most other works in the literature, Sections 2.1 and 2.2 provide a summary of some of the notions and of the results of [7]. Section 3 defines contact isotropic realisations and establishes their basic properties, while Section 4 tackles the problem of classifying contact isotropic realisations up to isomorphism. Throughout Sections 2-4, the case of Jacobi structures with trivial coefficients is presented as an example to illustrate the various notions introduced. The case of Poisson manifolds is considered in Section 5, which moreover compares symplectic and contact isotropic realisations. In particular, Section 5.2 interprets the main results of Sections 3 and 4 in the case in which the contact structures are co-oriented and the Jacobi structures are defined on trivial line bundles. For readers who are not familiar with the techniques for the general case, it may be helpful to read Section 5.2 alongside Sections 3 and 4. To make the transition to the general case, it is useful to think that many results follow from choosing local trivialisations of the line bundle, thus reducing the problem to the case of Jacobi structures over a trivial line bundle; the classical Spencer operator and the associated language can be viewed as a way to bypass the above approach. Furthermore, to make the exposition smoother, some longer or technical proofs of results used throughout the paper have been placed in appendices at the end. Appendix A deals with the geometric properties of the Jacobi manifolds that admit contact isotropic realisations. Appendices B and C provide proofs for some results of Section 2.3, and of Sections 4.2 and 4.3, respectively.

Throughout this note, there are two types of comments, labelled Note and Remark respectively; those with the former label are central to the problems studied in this paper, while those with the latter may be skipped at a first reading.

\footnotetext{
${ }^{1}$ The relation between contact isotropic realisations and integrable systems on contact manifolds is going to be explored in depth in a separate paper.
} 
Notation and conventions. Throughout the paper, the intersection of two subsets $U_{i} \cap U_{j}$ is denoted by $U_{i j}$. All line bundles considered in this paper are real unless otherwise stated.

\section{Basic notions and motivation}

The aim of this section is to recall fundamental notions regarding Jacobi structures, to establish notation, and to provide a family of motivating examples for the rest of the paper. Sections 2.1 and 2.2 follow the approach to contact and Jacobi manifolds of [7], which should be considered as the main reference for any detail missing below. More details regarding Jacobi structures and their properties can be found in $[9,12,18,23]$ amongst others.

\subsection{Jacobi structures: definitions and examples}

Definition 2.1. A Jacobi structure on a manifold $P$ is a pair $(L,\{\cdot, \cdot\})$ consisting of a line bundle $L \rightarrow P$, and a local Lie bracket $\{\cdot, \cdot\}: \Gamma(L) \times \Gamma(L) \rightarrow \Gamma(L)$, i.e.,

$$
\operatorname{supp}(\{u, v\}) \subset \operatorname{supp}(u) \cap \operatorname{supp}(v) \quad \forall u, v \in \Gamma(L),
$$

where $\operatorname{supp}(u)$ denotes the support of $u$. A Jacobi manifold is a triple $(P, L,\{\cdot, \cdot\})$, where $(L,\{\cdot, \cdot\})$ is a Jacobi structure on $P$.

Jacobi structures simultaneously generalise contact and Poisson structures.

Definition 2.2. A contact structure on a manifold $M$ is a smooth hyperplane distribution $H \subset T M$ whose curvature map

$$
c: H \times H \rightarrow T M / H,
$$

defined on sections by $c(X, Y):=[X, Y] \bmod H$ is fibre-wise non-degenerate. A contact manifold is a pair $(M, H)$, where $H$ is a contact structure on $M$.

Note 2.3. The majority of works in the literature on contact geometry concentrates on the case in which $H=\operatorname{ker} \theta$, for some 1 -form $\theta \in \Omega^{1}(M)$ whose differential $\mathrm{d} \theta$ makes $H \rightarrow M$ into a symplectic vector bundle. In this case the bundle $T M / H$ is trivial and the above map $c$ coincides with $\left.\mathrm{d} \theta\right|_{H}$. Such contact structures are henceforth referred to as being co-oriented. More generally, a contact structure $H$ on $M$ can be encoded equivalently as the kernel of the 1-form $\theta \in \Omega^{1}(M, L)$ given by projection onto $L:=T M / H$. This is henceforth referred to as the (canonical) generalised contact form of $(M, H)$. Throughout this paper, both points of views are used interchangeably.

The following family of examples of contact manifolds plays a prominent role throughout this paper.

Example 2.4. Let $\pi: L \rightarrow P$ a line bundle and (by abuse of notation) denote by $\pi: J^{1} L \rightarrow P$ the first jet bundle

$$
\left.J^{1} L\right|_{x}=\left\{j_{x}^{1} u \mid u \in \Gamma(L)\right\} .
$$

The Cartan contact form $\theta_{\text {can }} \in \Omega^{1}\left(J^{1} L, \mathrm{pr}^{*} L\right)$ defines a contact structure as in Note 2.3. It is defined by

$$
\theta_{\text {can }, j_{x}^{1} u}=D_{x}(\operatorname{pr}-u \circ \pi): T_{j_{x}^{1} u}\left(J^{1} L\right) \rightarrow L_{x},
$$

with pr: $J^{1} L \rightarrow L, j^{1} u_{x} \mapsto u(x)$, and where we have used the canonical identification $L_{x} \simeq$ $T_{u(x)}\left(L_{x}\right)$. The Cartan contact form detects holonomic sections of $J^{1} L$, i.e., those of the form $j^{1} u: x \mapsto j_{x}^{1} u$, for some $u \in \Gamma(L)$, in the sense that a section $\xi$ of $J^{1} L$ is holonomic if and only if $\xi^{*} \theta_{\text {can }}=0$. 
Example 2.5. A contact manifold $(M, H)$ comes equipped with a well-known natural Jacobi structure $(L=T M / H,\{\cdot, \cdot\})$ which can be described as follows (cf. $[7,10]$ and references therein). A Reeb vector field of $(M, H)$ is any vector field $R$ satisfying

$$
[R, \Gamma(H)] \subset \Gamma(H)
$$

the vector spaces of Reeb vector fields is denoted by $\mathfrak{X}_{\text {Reeb }}(M, H)$. The map

$$
\mathfrak{X}_{\text {Reeb }}(M, H) \rightarrow \Gamma(L), \quad R \mapsto \theta(R)
$$

is a vector space isomorphism (cf. [7, Lemma 2.2 and Corollary 2.3]). The inverse image of $u \in \Gamma(L)$ under the above isomorphism, denoted by $R_{u}$, is called the Reeb vector field associated to $u$. The space $\mathfrak{X}_{\text {Reeb }}(M, H)$ is closed under the Lie bracket of vector fields, hence it induces a Lie bracket on $\Gamma(L)$, explicitly given by

$$
\{u, v\}:=\left[R_{u}, R_{v}\right] \bmod H,
$$

for $u, v \in \Gamma(L)$. In the case in which $T M / H \rightarrow M$ is trivial, i.e., in the co-orientable case (cf. Note 2.3), the above Jacobi structure can be described easily as follows. Given a contact 1-form $\theta \in \Omega^{1}(M)$ with $H=\operatorname{ker} \theta$, let $R_{\theta} \equiv R_{1}$ be the Reeb vector field associated to (the function 1 and to) $\theta$. Then the space of Reeb vector fields is nothing but the space of Reeb vector fields associated to all contact forms defining $H$ and the induced bracket is well-known (cf. Example 2.6 below).

Example 2.6. A Poisson structure on a manifold $P$ is a bivector field $\Lambda \in \mathfrak{X}^{2}(P)$, which satisfies $\llbracket \Lambda, \Lambda \rrbracket=0$, where $\llbracket \cdot, \cdot \rrbracket$ is the Schouten bracket. We say that the pair $(P, \Lambda)$ is a Poisson manifold. In this case $\Lambda$ induces a Jacobi structure $\{\cdot, \cdot\}$ on the trivial line bundle $\mathbb{R}_{P}$ by observing that $\Lambda$ determines a local Lie bracket on the sections of the trivial bundle $\mathbb{R}_{P} \rightarrow P$. By abuse of notation, the induced Jacobi manifold is also denoted by $(P, \Lambda)$. More generally, a Jacobi structure $\left(\mathbb{R}_{P},\{\cdot, \cdot\}\right)$ on $P$ is completely determined by a pair $(\Lambda, R) \in \mathfrak{X}^{2}(P) \times \mathfrak{X}(P)$, satisfying

$$
\llbracket \Lambda, \Lambda \rrbracket=2 R \wedge \Lambda, \quad \llbracket \Lambda, R \rrbracket=0 .
$$

The Lie bracket on $\Gamma\left(\mathbb{R}_{P}\right)=C^{\infty}(P)$ is given by

$$
\{f, g\}:=\Lambda(\mathrm{d} f, \mathrm{~d} g)+f(R g)-g(R f)
$$

for $f, g \in C^{\infty}(P)($ cf. [26]).

Morphisms between Jacobi manifolds are defined as follows.

Definition 2.7. Let $\left(N, L_{N},\{\cdot, \cdot\}_{N}\right)$ and $\left(P, L_{P},\{\cdot, \cdot\}_{P}\right)$ be Jacobi manifolds such that there exists an isomorphism $F: \phi^{*} L_{P} \rightarrow L_{N}$. A map $\phi: N \rightarrow P$ is said to be Jacobi with bundle component $F$ if for all $u, v \in \Gamma\left(L_{P}\right)$

$$
\left\{F \circ \phi^{*} u, F \circ \phi^{*} v\right\}_{N}=F \circ \phi^{*}\{u, v\}_{P} .
$$

Remark 2.8. If the line bundles $L_{N}$ and $L_{P}$ are assumed to be trivial in Definition 2.7, then the above notion of Jacobi map with bundle component is often referred to in the literature as a conformal Jacobi morphism (cf. [12, Section 1.6]). 
Example 2.9. Let $(M, H)$ be a contact manifold with associated line bundle $L \rightarrow M$, where the notation is as in Example 2.5. The submanifold $L^{*} \backslash\{0\} \hookrightarrow T^{*} M$ is symplectic, where $T^{*} M$ is endowed with the canonical symplectic form (cf. [25, Definition 2.3]). Let $\Omega$ denote the symplectic form on $L^{*} \backslash\{0\}$; the symplectic manifold $\left(L^{*} \backslash\{0\}, \Omega\right)$ is said to be the symplectisation ${ }^{2}$ of $(M, H)$. The projection pr: $\left(L^{*} \backslash\{0\}, \Omega\right) \rightarrow(M, H)$ is a Jacobi map with bundle component $F_{\mathrm{pr}}: \operatorname{pr}^{*} L \rightarrow \mathbb{R}_{L^{*} \backslash\{0\}}$ given by $F_{\mathrm{pr}}(\alpha, u)=\alpha(X)$, where $u=X \bmod H$.

Example 2.10. Let $\mathfrak{g}^{*}$ denote the dual of a finite-dimensional real Lie algebra; endow it with the standard linear Poisson structure $\Lambda$. Its projectivisation $\mathbb{P}\left(\mathfrak{g}^{*}\right):=\frac{\mathfrak{g}^{*} \backslash\{0\}}{\mathbb{R}^{*}}$ admits a natural Jacobi structure $(O(1),\{\cdot, \cdot\})$, where $O(1) \rightarrow \mathbb{P}\left(\mathfrak{g}^{*}\right)$ is the dual of the tautological line bundle. The bracket $\{\cdot, \cdot\}$ on $\Gamma(O(1))$ can be defined as follows. Recall that $\Gamma(O(1))$ can be identified with the vector space of smooth homogeneous functions of degree 1 on $\mathfrak{g}^{*} \backslash\{0\}$, denoted by $\mathrm{C}_{h}^{\infty}\left(\mathfrak{g}^{*} \backslash\{0\}\right)$. Since $\mathfrak{g}^{*} \backslash\{0\} \subset \mathfrak{g}^{*}$ is an open subset which is the complement of a symplectic leaf, $\Lambda$ restricts to a Poisson structure on $\mathfrak{g}^{*} \backslash\{0\}$, also denoted by $\Lambda$. Linearity of $\Lambda$ implies that $\mathrm{C}_{h}^{\infty}\left(\mathfrak{g}^{*} \backslash\{0\}\right)$ is a Lie subalgebra of $\left(\mathrm{C}^{\infty}\left(\mathfrak{g}^{*} \backslash\{0\}\right),\{\cdot, \cdot\}_{\Lambda}\right)$. This defines a Lie bracket on $\Gamma(O(1))$ which is manifestly local and, hence, a Jacobi structure $(O(1),\{\cdot, \cdot\})$ as claimed. If $\mathbb{S}\left(\mathfrak{g}^{*}\right)=\frac{\mathfrak{g}^{*} \backslash\{0\}}{\mathbb{R}^{+}}$, the covering map $q: \mathbb{S}\left(\mathfrak{g}^{*}\right) \rightarrow \mathbb{P}\left(\mathfrak{g}^{*}\right)$ can be used to define a Jacobi structure $\left(q^{*}(O(1)),\{\{\cdot, \cdot\}\}\right)$. The vector bundle $q^{*}(O(1)) \rightarrow \mathbb{S}\left(\mathfrak{g}^{*}\right)$ is trivialisable and a trivialisation can be obtained by picking a metric on $\mathfrak{g}$; once such a choice is made, this example recovers the Jacobi structures considered in $[13,26]$, and [33, Example 2.3]. Finally, the natural projection $\pi:\left(\mathfrak{g}^{*} \backslash\{0\}, \Lambda\right) \rightarrow\left(\mathbb{P}\left(\mathfrak{g}^{*}\right), O(1),\{\cdot, \cdot\}\right)$ is a Jacobi map with bundle component $F_{\pi}: \pi^{*}(O(1)) \rightarrow \mathbb{R}_{\mathfrak{g}^{*} \backslash\{0\}}$, given by $F_{\pi}(x, \eta)=\eta(x)$.

\subsection{The Lie algebroid and Spencer operator of a Jacobi manifold}

In analogy with Poisson manifolds, the geometric structure of a Jacobi manifold can be completely encoded by a Lie algebroid together with a Spencer operator (cf. [7, 8, 10, 22]). In general, a Lie algebroid over a manifold $M$ is a vector bundle $\mathrm{A} \rightarrow M$, together with a Lie bracket $[\cdot, \cdot]$ on $\Gamma(\mathrm{A})$, and a vector bundle map $\rho: \mathrm{A} \rightarrow T M$, called the anchor map, satisfying the compatibility condition

$$
[\alpha, f \beta]=f[\alpha, \beta]+L_{\rho(\alpha)}(f) \beta
$$

for all $f \in C^{\infty}(M)$, and all $\alpha, \beta \in \Gamma(\mathrm{A})$. Given a Jacobi manifold $(P, L,\{\cdot, \cdot\})$, the first jet bundle $J^{1} L \rightarrow P$ can be endowed with the structure of a Lie algebroid as follows (cf. [7] for a proof).

Proposition 2.11. Given a Jacobi manifold $(P, L,\{\cdot, \cdot\})$, there exists a Lie algebroid structure on $J^{1} L \rightarrow P$ which is uniquely characterised by the following properties:

(I) The anchor map $\rho: J^{1} L \rightarrow T P$ satisfies

$$
\{u, f v\}=f\{u, v\}+L_{\rho\left(j^{1} u\right)}(f) v,
$$

for all $u, v \in \Gamma(L), f \in C^{\infty}(P)$;

(II) The Lie bracket on $\Gamma\left(J^{1} L\right)$ satisfies

$$
\left[j^{1} u, j^{1} v\right]=j^{1}\{u, v\}, \quad \forall u, v \in \Gamma(L) .
$$

This Lie algebroid is henceforth referred to as the associated Lie algebroid to $(P, L,\{\cdot, \cdot\})$.

\footnotetext{
${ }^{2}$ This notion differs slightly with that in the literature on co-orientable contact structures, where a connected component of $L^{*} \backslash\{0\}$ is declared to be the symplectisation of $(M, H)$.
} 
Note 2.12. If $L=\mathbb{R}_{P}$, the above Lie algebroid agrees with that defined in [9, Definition 1.4], where the identification $j_{x}^{1} f \mapsto\left(\mathrm{d}_{x} f, f(x)\right)$ of $J^{1} \mathbb{R}_{P}$ with $\mathrm{T}^{*} P \times \mathbb{R}$ is used. In this case the anchor $\rho: T^{*} P \times \mathbb{R} \rightarrow T P$ becomes

$$
\rho(\omega, \lambda)=\Lambda^{\sharp}(\omega)+\lambda R,
$$

where $(\Lambda, R)$ are as in Example 2.6. In particular, if the underlying structure is Poisson (i.e., $R=0$ ), then the kernel of the anchor contains $0 \oplus \mathbb{R}$.

Note 2.13. The condition for a map to be Jacobi can be formulated in terms of anchors of Lie algebroids. Suppose that $\phi:\left(N, L_{N},\{\cdot, \cdot\}_{N}\right) \rightarrow\left(P, L_{P},\{\cdot, \cdot\}_{P}\right)$ is a Jacobi map with bundle component $F$, then the following diagram commutes

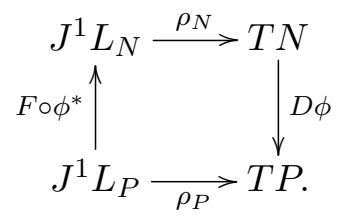

Remark 2.14. The image of the anchor of a Lie algebroid defines a (singular) foliation on the base manifold. Unlike what happens in Poisson geometry, the leaves of (the foliation induced by the Lie algebroid associated to) a Jacobi manifold may be even or odd dimensional. If the induced foliation is regular, i.e., integrable in the sense of Frobenius, the underlying Jacobi manifold is said to be regular.

In analogy with what happens for Poisson manifolds, the Lie algebroid associated to a Jacobi manifold comes with compatible extra structure. Fix any line bundle $L \rightarrow P$ (not necessarily with a Jacobi bracket); its first jet bundle $J^{1} L$ fits into a short exact sequence of vector bundles

$$
0 \rightarrow \mathrm{T}^{*} P \otimes L \stackrel{i}{\rightarrow} J^{1} L \stackrel{\mathrm{pr}}{\rightarrow} L \rightarrow 0
$$

where $i(d f \otimes u)=f j^{1}(u)-j^{1}(f u)$ for $f \in C^{\infty}(P)$ and $u \in \Gamma(L)$. While the above sequence is not canonically split, the map $u \mapsto j^{1} u$ gives a canonical splitting at the level of sections:

$$
\Gamma\left(J^{1} L\right) \cong \Gamma(L) \oplus \Omega^{1}(P ; L),
$$

known as the Spencer decomposition.

Definition 2.15. The classical Spencer operator associated to $L \rightarrow P$ is the projection D: $\Gamma\left(J^{1} L\right) \rightarrow \Omega^{1}(P ; L)$.

Example 2.16. Suppose that $L=\mathbb{R}_{P}$, i.e., it is trivial; then using the identification of $J^{1} \mathbb{R}_{P} \cong$ $\mathrm{T}^{*} P \oplus \mathbb{R}_{P}$ of Note 2.12 , identify an element of $\Gamma\left(J^{1} \mathbb{R}_{P}\right)$ with a pair $(\eta, f)$, where $\eta \in \Omega^{1}(P)$ and $f \in C^{\infty}(P)$. Then the classical Spencer operator is given by $\mathrm{D}(\eta, f)=\mathrm{d} f-\eta$.

Note 2.17. The classical Spencer operator D associated to $L \rightarrow P$ is completely determined by the following two conditions:

- for any $u \in \Gamma(L), \mathrm{D}\left(j^{1} u\right)=0$;

- for any $X \in \mathfrak{X}(P), \alpha \in \Gamma\left(J^{1} L\right)$ and $f \in C^{\infty}(P)$,

$$
\mathrm{D}_{X}(f \alpha)=f \mathrm{D}_{X}(\alpha)+d f(X) \operatorname{pr}(\alpha),
$$

i.e., the Leibniz identity holds. 
Moreover, the $C^{\infty}(P)$-module structure on $\Gamma\left(J^{1} L\right)$ induced by the Spencer decomposition is the following

$$
f \cdot(u, \phi)=(f u, \phi+d f \otimes u),
$$

for any $f \in C^{\infty}(P), u \in \Gamma(L)$ and $\phi \in \Omega^{1}(P ; L)$. Finally, the classical Spencer operator associated to $L \rightarrow P$ is related to the Cartan contact form $\theta_{\text {can }}$ on $J^{1} L$ (cf. Example 2.4) as follows. If $\alpha \in \Gamma\left(J^{1} L\right)$, then

$$
\mathrm{D}(\alpha)=\alpha^{*} \theta_{\text {can }} .
$$

Suppose that $(P, L,\{\cdot, \cdot\})$ is a Jacobi manifold. In this case, the classical Spencer operator is compatible with the Lie algebroid structure on $J^{1} L \rightarrow P$ defined in Proposition 2.11 in the following sense. The Jacobi bracket defines a flat $J^{1} L$-connection on $L \rightarrow P$, i.e., an operator $\nabla: \Gamma\left(J^{1} L\right) \times \Gamma(L) \rightarrow \Gamma(L)$ which is $C^{\infty}(P)$-linear in the first component, satisfies the Leibniz identity $\nabla_{\alpha}(f u)=f \nabla_{\alpha}(u)+L_{\rho(\alpha)}(f) u$, and the flatness equation $\nabla_{[\alpha, \beta]}=\nabla_{\alpha} \nabla_{\beta}-\nabla_{\beta} \nabla_{\alpha}$, for any $f \in C^{\infty}(P), \alpha, \beta \in \Gamma\left(J^{1} L\right)$ and $u \in \Gamma(L)$. It is uniquely defined by the formula

$$
\nabla: \Gamma\left(J^{1} L\right) \times \Gamma(L) \rightarrow \Gamma(L), \quad \nabla_{j^{1} u}(v)=\{u, v\} .
$$

The compatibility of $\mathrm{D}$ with the Lie algebroid structure on $J^{1} L \rightarrow P$ can be encoded in the following two equalities

$$
\begin{aligned}
& \mathrm{D}_{\rho(\alpha)}\left(\alpha^{\prime}\right)=\nabla_{\alpha^{\prime}}(\operatorname{pr}(\alpha))+\operatorname{pr}\left(\left[\alpha, \alpha^{\prime}\right]\right), \\
& \mathrm{D}_{X}\left[\alpha, \alpha^{\prime}\right]=\nabla_{\alpha}\left(\mathrm{D}_{X} \alpha^{\prime}\right)-\mathrm{D}_{[\rho(\alpha), X]} \alpha^{\prime}-\nabla_{\alpha^{\prime}}\left(\mathrm{D}_{X} \alpha\right)+\mathrm{D}_{\left[\rho\left(\alpha^{\prime}\right), X\right]} \alpha,
\end{aligned}
$$

for any $\alpha, \alpha^{\prime} \in \Gamma\left(J^{1} L\right)$ and $X \in \mathfrak{X}(P)$ (cf. [8] for a general notion of Spencer operators). Henceforth, the classical Spencer operator D associated to a line bundle $L \rightarrow P$ endowed with a Jacobi bracket is referred to as the Spencer operator associated to the Jacobi manifold $(P, L,\{\cdot, \cdot\})$.

\subsection{Motivating example: Multiplicity-free actions on contact manifolds}

Throughout this section, let $G$ denote a compact connected Lie group, and denote symplectic and contact manifolds by $(S, \omega)$ and $(M, H)$ respectively. If $\mathfrak{g}=\operatorname{Lie}(G), G$ acts on $\mathfrak{g}^{*}$ by the coadjoint action, which is henceforth understood to be the $G$-action on $\mathfrak{g}^{*}$. Proofs of the main results of this subsection can be found in Appendix B. First the most basic notions of symmetry in symplectic and contact geometry are recalled for completeness.

\section{Definition 2.18.}

- An action $G \curvearrowright(S, \omega)$ is said to be Hamiltonian if there exists a smooth $G$-equivariant map $\chi: S \rightarrow \mathfrak{g}^{*}$, called moment map, such that for all $\xi \in \mathfrak{g}=\operatorname{Lie}(G)$,

$$
\omega\left(X_{\xi},-\right)=\mathrm{d}\langle\chi, \xi\rangle,
$$

where $X_{\xi}$ is the vector field on $M$ induced by $\xi$ and $\langle\cdot, \cdot\rangle$ denotes the standard pairing between $\mathfrak{g}$ and $\mathfrak{g}^{*}$.

- An action $G \curvearrowright(M, H)$ is said to be contact if it preserves the contact structure $H$, i.e., for all $g \in G, p \in M, D_{p} g\left(H_{p}\right)=H_{g \cdot p}$.

Note 2.19. Suppose that $G \curvearrowright(M, H)$ is contact. Its lift to the cotangent bundle $G \curvearrowright$ $\left(T^{*} M, \omega_{\text {can }}\right)$ defined by $(g \cdot \alpha)(X)=\alpha\left(D g^{-1}(X)\right)$, where $\alpha \in T^{*} M$ and $X \in T M$, is Hamiltonian with moment map $\mu: T^{*} M \rightarrow \mathfrak{g}^{*}$ given by $\mu(\alpha)(\xi)=\alpha\left(D \operatorname{pr}\left(X_{\xi}\right)\right)$, where $\xi \in \mathfrak{g}, X_{\xi}$ is the vector field on $T^{*} M$ by $\xi$, and $\operatorname{pr}: T^{*} M \rightarrow M$ is the projection. This action preserves $L^{*} \backslash\{0\}$, thus inducing a Hamiltonian action on the symplectisation $\left(L^{*} \backslash\{0\}, \Omega\right)$ whose moment map $\mu: L^{*} \backslash\{0\} \rightarrow \mathfrak{g}^{*}$ is $\mathbb{R}^{*}$-equivariant, i.e., for all $t \in \mathbb{R}^{*}$ and all $\alpha \in L^{*} \backslash\{0\}, \mu(t \alpha)=t \mu(\alpha)$. 
For the purposes at hand, it is useful to recall some simple notions associated to Lie group actions.

Definition 2.20. An action of $G$ on a manifold $N$ is said to be

- locally free at $p \in N$ if the stabiliser $G_{p}:=\{g \in G \mid g \cdot p=p\}$ is discrete;

- effective if $\bigcap_{p \in N} G_{p}=\{e\}$.

The following definition introduces a notion of 'integrable' group actions of compact Lie groups on symplectic and contact manifolds (cf. [19, Definition 5.1.11] for the symplectic case and [25, Definition 2.11] for torus actions on contact manifolds).

Definition 2.21. Let $G$ be a compact Lie group. An action $G \curvearrowright(S, \omega)$ (respectively $G \curvearrowright$ $(M, H))$ is said to be multiplicity-free if it is Hamiltonian (respectively contact), effective, locally free at some point, and if $\operatorname{dim} S=\operatorname{dim} G+\operatorname{rk} G$ (respectively $\operatorname{dim} M=\operatorname{dim} G+\operatorname{rk} G-1$ ), where rk $G$ denotes the rank of $G$, i.e., the dimension of its maximal torus.

In the context of Hamiltonian actions of compact Lie groups, multiplicity-free actions can be thought of as being 'integrable'. More precisely, if a compact Lie group $G$ acts on $(S, \omega)$ so that the action is locally free at some point, then

$$
\operatorname{dim} S \geq \operatorname{dim} G+\operatorname{rk} G
$$

(cf. [19, Theorem 5.1.6]). The condition of being multiplicity-free corresponds to considering the case in which $\operatorname{dim} S$ is completely determined by the group acting (e.g., when $G=\mathbb{T}^{n}$, then $\operatorname{dim} S=2 n$ ). Moreover, the following lemma illustrates the relation between the notions of multiplicity-free actions in contact and symplectic geometry by considering symplectisations.

Lemma 2.22. If a contact action $G \curvearrowright(M, H)$ is multiplicity-free then the induced Hamiltonian action on $\left(L^{*} \backslash\{0\}, \Omega\right)$ is too.

Proof. To show that the induced action on $\left(L^{*} \backslash\{0\}, \Omega\right)$ is multiplicity-free, it suffices to check that the action is locally free at some point and effective, as the condition on the dimension of $L^{*} \backslash\{0\}$ and on $G$ is automatically satisfied. Both claims follow by observing that the projection pr: $L^{*} \backslash\{0\} \rightarrow M$ is $G$-equivariant, thus implying that, for all $\alpha \in L^{*} \backslash\{0\}$, $G_{\alpha} \subset G_{\operatorname{pr}(\alpha)}$.

Example 2.23. Let $G$ be a (compact) simply connected Lie group acting on a closed symplectic manifold $(S, \omega)$ in a multiplicity-free fashion. Denote the moment map by $\chi:(S, \omega) \rightarrow \mathfrak{g}^{*}$ and, for each $\xi \in \mathfrak{g}$, set $\chi^{\xi}:=\langle\chi, \xi\rangle: S \rightarrow \mathbb{R}$. Suppose further that $\omega$ is integral and consider the prequantum circle bundle $\phi:(M, H) \rightarrow(S, \omega)$ as in Example 3.4. The infinitesimal $\mathfrak{g}$-action on $S$ can be lifted to an infinitesimal $\mathfrak{g}$-action on $M$, by setting $\xi \mapsto R_{\phi^{*}} \chi^{\xi}$, where $R_{\phi^{*} \chi^{\xi}}$ is the Reeb vector field associated to the function $\phi^{*} \chi^{\xi}$. This holds because $\phi:(M, H) \rightarrow(S, \omega)$ is a contact (isotropic) realisation (cf. Definitions 3.1 and 3.2). Since $M$ is compact, this action can be integrated to a $G$-action on $M$ which is, in fact, contact; moreover, $\phi$ is $G$-equivariant. This $G$-action commutes with the (contact!) $S^{1}$-action coming from the principal $S^{1}$-bundle structure on $\phi: M \rightarrow S$; this follows because the functions $\phi^{*} \chi^{\xi}$ are basic. This yields a contact $G \times S^{1}$-action on $(M, H)$ which is multiplicity-free since the $G$-action on $(S, \omega)$ is and because $\phi:(M, H) \rightarrow(S, \omega)$ is $G$-equivariant.

Example 2.24. Not all examples of multiplicity-free actions in contact geometry arise as in Example 2.23. For instance, consider $M=S^{3} \cong \mathrm{SU}(2)$ endowed with a left-invariant contact structure $H$ (cf. [2]). The $\mathrm{SU}(2)$-action on $(M, H)$ given by left-translations is multiplicity-free and cannot arise as in Example 2.23 because $\mathrm{SU}(2)$ is simply-connected. 
Multiplicity-free actions on contact manifolds satisfy a strong property which generalises [25, Lemma 2.12].

Proposition 2.25. Let $G \curvearrowright(M, H)$ be a multiplicity-free action and denote the moment map of the induced Hamiltonian action on $\left(L^{*} \backslash\{0\}, \Omega\right)$ by $\mu: L^{*} \backslash\{0\} \rightarrow \mathfrak{g}^{*}$. Then $\mu\left(L^{*} \backslash\{0\}\right) \subset \mathfrak{g}^{*} \backslash\{0\}$.

The proof of Proposition 2.25 is postponed to Appendix B. An immediate consequence of Proposition 2.25 and Note 2.19 is the following.

Corollary 2.26. Associated to a multiplicity-free action $G \curvearrowright(M, H)$ is a smooth map $\phi: M \rightarrow$ $\mathbb{P}\left(\mathfrak{g}^{*}\right)$ which makes the following diagram commutative

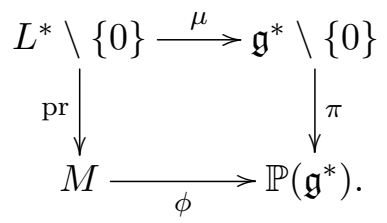

This motivates introducing the following notion.

Definition 2.27. Given a multiplicity-free action $G \curvearrowright(M, H)$, the map $\phi: M \rightarrow \mathbb{P}\left(\mathfrak{g}^{*}\right)$ as in Corollary 2.26 is called the Jacobi moment map associated to the action.

Example 2.28. Fix notation as in Example 2.23. Suppose that $G \times S^{1} \curvearrowright(M, H)$ is multiplicityfree, where $(M, H)$ is the total space of a principal $S^{1}$-bundle of a closed symplectic manifold $(S, \omega)$ endowed with a multiplicity-free action of the compact, simply connected Lie group $G$. The symplectisation of $(M, H)$ can be written as $\left(M \times \mathbb{R}^{*}, \mathrm{~d}(t \alpha)\right)$, where $\alpha \in \Omega^{1}(M)$ is a $G \times$ $S^{1}$-invariant contact form. The moment map for the lifted Hamiltonian $G \times S^{1}$-action on $\left(M \times \mathbb{R}^{*}, \mathrm{~d}(t \alpha)\right)$ can be written as $\mu(m, t)=(t \mu(\Phi(m)), t) \in \mathfrak{g}^{*} \times \mathbb{R}$, where $\Phi: M \rightarrow S$ is the projection and $\mu: S \rightarrow \mathfrak{g}^{*}$ is the moment map of the Hamiltonian $G$-action. Since $t \in \mathbb{R}^{*}$ in the above formula, it follows that the image of $\mu$ does not contain $(0,0) \in \mathfrak{g} \times \mathbb{R}$. In this case, the Jacobi moment map $\phi: M \rightarrow \mathbb{P}\left(\mathfrak{g}^{*} \times \mathbb{R}\right)$ can be written as $\phi(m)=[\mu(\Phi(m)): 1] \in \mathbb{P}\left(\mathfrak{g}^{*} \times \mathbb{R}\right)$.

The next theorem illustrates properties of the Jacobi moment map, which serve as motivation for the rest of this paper (cf. [33, Proposition 3.4, Lemma 3.5 and Proposition 5.1] for statements analogous to (J1) and (J2) in the case of co-oriented contact manifolds).

Theorem 2.29. The Jacobi moment map $\phi:(M, H) \rightarrow\left(\mathbb{P}\left(\mathfrak{g}^{*}\right), O(1),\{\cdot, \cdot\}\right)$ associated to a multiplicity-free contact action $G \curvearrowright(M, H)$ is Jacobi with bundle component $F_{\phi}: \phi^{*}(O(1)) \rightarrow L$, where $F_{\phi}(p, \eta) \in L_{p}$ is the unique element such that, for all $\alpha \in L_{p}^{*} \backslash\{0\}, \eta(\mu(\alpha))=\alpha\left(F_{\phi}(p, \eta)\right)$, and $\mu: L^{*} \backslash\{0\} \rightarrow \mathfrak{g}^{*}$ is the moment map of the lifted action. Moreover, if $M_{\text {prin }} \subset M$ denotes the open, dense subset consisting of points whose stabiliser is discrete, then for all $p \in M_{\text {prin }}$,

(J1) $D_{p} \phi$ is onto;

(J2) ker $D_{p} \phi$ is transversal to the contact distribution, i.e.,

$$
\text { ker } D_{p} \phi+H_{p}=T_{p} M
$$

(J3) $\operatorname{ker} D_{p} \phi \subset\left(\operatorname{ker} D_{p} \phi\right)^{\perp}$, where $\left(\operatorname{ker} D_{p} \phi\right)^{\perp}=\rho\left(F_{\phi}\left(\phi^{*}\left(J^{1} O(1)\right)\right)\right)$ and $\rho: J^{1} L \rightarrow T M$ is the anchor map of the Lie algebroid associated to $(M, H)$.

In light of Corollary 2.26 and Theorem 2.29, the theory of multiplicity-free actions on contact manifolds brings about Jacobi maps satisfying properties (J1)-(J3). The rest of the paper concentrates on studying properties of such maps; this can be seen as both a first step towards understanding properties of multiplicity-free actions in the contact setting and a generalisation of the aforementioned actions. 


\section{Contact isotropic realisations of Jacobi manifolds}

This paper studies the classification of a special type of contact realisations of Jacobi manifolds (cf. [33, Definition 3.7]), called contact isotropic realisations (or CIR for short). The aim of this section is to define such realisations and to give their basic properties.

Definition 3.1. A contact realisation of a Jacobi manifold $(P, L,\{\cdot, \cdot\})$ is a contact manifold $(M, H)$, together with a surjective submersion $\phi:(M, H) \rightarrow(P, L,\{\cdot, \cdot\})$ satisfying the following properties:

(CR1) $\phi$ is a Jacobi map with bundle component $F: \phi^{*}(L) \rightarrow L_{M}$, where $L_{M}:=T M / H$ (cf. Definition 2.7)

(CR2) $H=\operatorname{ker} \theta$ is transversal to $\phi$, i.e.,

$$
H+\operatorname{Ker} D \phi=\mathrm{T} M \text {. }
$$

Henceforth, whenever referring to a contact realisation $\phi:(M, H) \rightarrow(P, L,\{\cdot, \cdot\})$, the dimensions of $M$ and of $P$ are fixed to be $2 n+1$ and $2 n+1-k$ respectively.

Definition 3.2. A contact isotropic realisation (CIR for short) of a Jacobi manifold $(P, L,\{\cdot, \cdot\}$ ) is a contact realisation $\phi:(M, H) \rightarrow(P, L,\{\cdot, \cdot\})$ with connected, compact fibers satisfying the isotropic condition:

(I) $\operatorname{Ker} D \phi \subset(\operatorname{Ker} D \phi)^{\perp}$, where $(\operatorname{Ker} D \phi)^{\perp}:=\rho_{M}\left(F\left(\phi^{*} J^{1} L\right)\right)$ is the pseudo-orthogonal distribution of ker $D \phi$.

The isomorphism $F$ is henceforth assumed to be the identity to simplify the notation and the exposition.

Note 3.3. For Jacobi manifolds with trivial bundle component $L=\mathbb{R}_{P}$, condition (I) is equivalent to requiring that the subbundle ker $D \phi \cap H \subset H$ is isotropic. This can be seen directly by using property (I), and the description of $H=\operatorname{ker} \theta$, by a contact form $\theta \in \Omega^{1}(M)$ (which trivialises $T M / H)$. In this case, the Jacobi structure $\left(\Lambda_{M}, R_{\theta}\right)$ is determined by $\theta$ and its Reeb vector field $R_{\theta}$ (cf. Example 2.5), and $-c^{\sharp}:=\Lambda_{M}^{\sharp}: T^{*} M \rightarrow H \subset T M$ is uniquely defined by the equations

$$
i_{\Lambda_{M}^{\sharp}(\omega)} \theta=0, \quad i_{\Lambda_{M}^{\sharp}(\omega)} \mathrm{d} \theta=-\omega+\omega\left(R_{\theta}\right) \theta
$$

(see Note (2.12)). Thus,

$$
(\operatorname{Ker} D \phi)^{\perp}=\rho_{M}\left(\phi^{*}\left(T^{*} P\right) \times \mathbb{R}\right)=\Lambda_{M}^{\sharp}\left((\operatorname{ker} D \phi)^{\circ}\right) \oplus \mathbb{R}\left\langle R_{\theta}\right\rangle=(D \phi \cap H)^{\perp} \oplus \mathbb{R}\left\langle R_{\theta}\right\rangle,
$$

where $(\operatorname{ker} D \phi)^{\circ} \subset T^{*} M$ denotes the annihilator of ker $D \phi$, and, by abuse of notation, $(D \phi \cap H)^{\perp}$ denotes the symplectic orthogonal of $D \phi \cap H$ in $(H, \mathrm{~d} \theta)$. This explains the terminology used in Definition 3.2.

Example 3.4 (prequantum circle bundles over symplectic manifolds). Let $(S, \omega)$ be a symplectic manifold whose symplectic form $\omega$ is integral, i.e., it defines a cohomology class $[\omega] \in \mathrm{H}^{2}(S ; \mathbb{Z})$. It is well-known that the total space of the principal $S^{1}$-bundle $\phi: M \rightarrow S$ with Chern class $[\omega]$ admits a contact structure $H=\operatorname{ker} \theta$, where $\theta \in \Omega^{1}(M)$ is a connection 1-form whose curvature is $\omega$ (cf. [16, Section 7.2] and [28, Example 3.48]). Using Note (3.3), one can check that prequantum circle bundles are examples of CIR. 
Example 3.5 (contact non-commutative integrable systems, cf. [21]). Given a contact manifold $(M, H)$, say that $X \in \mathfrak{X}(M)$ is an infinitesimal automorphism of $(M, H)$ if it is a Reeb vector field (cf. Example 2.5). Fix a contact manifold $(M, H)$ and an infinitesimal automorphism $X$ of $(M, H)$. Following [21, Section 5.1], the dynamical system $\dot{x}(t)=X(x(t))$ is said to be (nonHamiltonian) completely integrable if there exists an open, dense subset $M_{\text {reg }} \subset M$, a proper surjective submersion $\phi: M_{\text {reg }} \rightarrow P$, and an abelian Lie algebra $\mathcal{X}$ of infinitesimal symmetries of $(M, H)$ such that $X$ is tangent to the fibres of $\phi$ and the latter are the orbits of $\mathcal{X}$. Moreover, such a system is said to be contact non-commutative (Hamiltonian) integrable if the foliation induced by $\phi$ is transversal to $\mathcal{H}$ and satisfies property (I) (cf. [21, Definitions 2 and 3]). Contact non-commutative ntegrable systems (with connected fibres) can be viewed as examples of CIRs: [21, Theorem 3] implies that if $\phi: M_{\mathrm{reg}} \rightarrow P$ is the associated proper surjective submersion, then there exists a unique line bundle $L \rightarrow P$, a unique bundle map $F: \phi^{*}(L) \rightarrow L_{M}$, and a unique Jacobi structure $\{\cdot, \cdot\}$ making $\phi:(M, H) \rightarrow(P, L,\{\cdot, \cdot\})$ satisfy property (CR1).

The existence of a CIR imposes geometric restrictions on the underlying Jacobi manifold, as illustrated below.

Lemma 3.6. If $\phi:(M, H) \rightarrow(P, L,\{\cdot, \cdot\})$ is a CIR, then the Jacobi structure on $P$ is regular with corank equal to $k=\operatorname{dim} \operatorname{ker} D \phi$, and all its leaves are even dimensional.

Proof. Fix points $p \in P$ and $m \in M$ with $m \in \phi^{-1}(p)$. By property (CR1) and Note 2.13, have that

$$
\operatorname{rk} \rho_{p}\left(J_{p}^{1}(L)\right)=\operatorname{rk}\left(\rho_{M, m} \circ \phi^{*}\left(J^{1} L\right)\right)-\operatorname{rk} \operatorname{ker}\left(\left.D \phi\right|_{\rho_{M, m} \circ \phi^{*}\left(J^{1} L\right)}\right) .
$$

On the one hand, property (CR2) implies that $\rho_{M} \circ \phi^{*}: J^{1} L \rightarrow T M$ is injective: indeed, if $c: H \times H \rightarrow L$ is the curvature map of equation (2.1), it can be shown that for $(\bar{u}, \bar{\eta}) \in$ $\Gamma\left(L_{M}\right) \oplus \Omega^{1}\left(M ; L_{M}\right) \simeq \Gamma\left(J^{1} L_{M}\right)$,

$$
\rho_{M}(\bar{u}, \bar{\eta})=R_{\bar{u}}-c^{\sharp}\left(\left.\bar{\eta}\right|_{H}\right) .
$$

Hence, if $\rho_{M}\left(\phi^{*} u, \phi^{*} \eta\right)=0$, for $u \in \Gamma(L)$ and $\eta \in \Omega^{1}(P ; L)$, then $R_{\left(\phi^{*} u\right)}(m)=c^{\sharp}\left(\circ\left(\left.\phi^{*} \eta\right|_{H}\right)\right)(m)$ $\in H$. By definition of Reeb vector fields, this means that

$$
\left(\phi^{*} u\right)(m)=R_{\left(\phi^{*} u\right)}(m) \bmod H=0,
$$

implying in turn that $\left.\phi^{*} \eta_{m}\right|_{H}=0$. Condition (CR2) gives that $\left.D \phi\right|_{H_{m}}: H_{m} \rightarrow T_{\phi(m)} P$ is surjective (as $\phi$ is a surjective submersion); thus $\left.\phi^{*} \eta_{m}\right|_{H}=0$ holds if and only if $\eta_{\phi(m)}=0$, thus proving injectivity of $\rho_{M} \circ \phi^{*}$.

Condition (I) gives that $\operatorname{rk} \operatorname{ker}\left(\left.D \phi\right|_{\rho_{M} \circ \phi^{*} J^{1} L}\right)=\operatorname{rk} \operatorname{ker} D \phi=k$. By definition $\operatorname{rk} J^{1} L=$ $2 n-k+2$, while injectivity of $\rho_{M} \circ \phi^{*}$ implies that

$$
\operatorname{rk} \rho_{M, m}\left(\phi^{*} J^{1} L\right)=2 n-k+2 ;
$$

equation (3.1) yields $\left.\operatorname{rk} \rho_{P, p}\left(J_{p}^{1}(L)\right)\right)=2 n+2-k-k=2 n+2-2 k$. Since $p \in P$ is arbitrary, the proof of the lemma is completed.

Remark 3.7. Suppose that $G \curvearrowright(M, H)$ is multiplicity-free (cf. Definition 2.21); by assumption $G$ is compact, thus implying that its Lie algebra $\mathfrak{g}$ is of compact type. Thus all symplectic leaves of the linear Poisson structure $\Lambda$ on $\mathfrak{g}^{*}$ are compact, as these are precisely coadjoint orbits. Moreover, since they are contained in the level set of a quadratic Casimir, they are transversal to the Euler vector field $E \in \mathfrak{X}^{1}\left(\mathfrak{g}^{*} \backslash\{0\}\right)$. By [12, Remarque 2.6], this implies that all leaves of the Jacobi manifold $\left(\mathbb{P}\left(\mathfrak{g}^{*}\right), O(1),\{\cdot, \cdot\}\right)$ of Example 2.10 are even dimensional. 
Note 3.8. Suppose that $(P, L,\{\cdot, \cdot\})$ is regular and that all of its leaves are even dimensional; denote the induced foliation by $\mathcal{F}$. Then

- the kernel of the anchor of $J^{1} L \rightarrow P$ is a bundle of abelian Lie algebras fitting in a short exact sequence of vector bundles

$$
0 \rightarrow \nu^{*} \otimes L \rightarrow \operatorname{ker} \rho \rightarrow L \rightarrow 0
$$

where $\nu^{*}=(T \mathcal{F})^{\circ}$ is the conormal bundle to $\mathcal{F}$;

- there is a canonical foliated 2-form $\omega_{\mathcal{F}} \in \Omega^{2}(\mathcal{F} ; L)$ uniquely defined by

$$
\omega_{\mathcal{F}}\left(\rho\left(j^{1} u\right), \rho\left(j^{1} v\right)\right):=\{u, v\},
$$

for any $u, v \in \Gamma(L)$. An explicit form for $\omega$ is

$$
\omega_{\mathcal{F}}(\rho(u, \eta), \rho(v, \zeta)):=\{u, v\}+\eta(\rho(v, \zeta))-\zeta(\rho(u, \eta)),
$$

for $(u, \eta),(v, \zeta) \in \Gamma\left(J^{1} L\right)$;

- the form $\omega_{\mathcal{F}}$ is closed with respect to the differential $\mathrm{d}_{\mathcal{F}}$ on the complex $\Omega^{*}(\mathcal{F} ; L)$ associated to the induced flat connection

$$
\bar{\nabla}: \Gamma(T \mathcal{F}) \times \Gamma(L) \rightarrow \Gamma(L)
$$

on $L$ uniquely defined by

$$
\bar{\nabla}_{\rho\left(j^{1} u\right)} v:=\nabla_{j^{1} u} v=\{u, v\}
$$

for any $u, v \in \Gamma(L)$. Explicitly, the differential $\mathrm{d}_{\mathcal{F}}$ is given on a foliated $k$-form $\omega$ by the standard Koszul-type formula

$$
\begin{aligned}
\mathrm{d}_{\mathcal{F}}(\omega)\left(X_{0}, \ldots, X_{k}\right)= & \sum_{i}(-1)^{i} \bar{\nabla}_{X_{i}}\left(\omega\left(X_{0}, \ldots, \hat{X}_{i}, \ldots, X_{k}\right)\right) \\
& +\sum_{i<j}(-1)^{i+j} \omega\left(\left[X_{i}, X_{j}\right], X_{0}, \ldots, \bar{X}_{i}, \ldots, \bar{X}_{j}, \ldots, X_{k}\right) .
\end{aligned}
$$

The foliated cohomology class $\left[\omega_{\mathcal{F}}\right] \in H^{2}(\mathcal{F} ; L)$ plays a central role in the classification of CIR of a fixed Jacobi manifold (cf. Theorem 4.18).

Proofs of the above properties can be found in Appendix A.

Example 3.9. The properties of regular Jacobi manifolds listed in Note 3.8 resemble closely those satisfied by Poisson manifolds; in fact, the former generalise the latter, as illustrated below. Let $(P, \Lambda)$ be a regular Poisson manifold, with symplectic foliation $\mathcal{F}$ and conormal bundle denoted by $\nu^{*}$. The corresponding Jacobi structure is defined on $L:=\mathbb{R}_{P}$ and the anchor $\rho:=J^{1} P=T^{*} P \oplus \mathbb{R} \rightarrow T P$ is given by $\rho(\eta, c)=\Lambda^{\sharp}(\eta)$ (cf. Example 2.6). Thus

$$
\text { ker } \rho=\nu^{*} \oplus \mathbb{R} \subset \mathrm{T}^{*} P \oplus \mathbb{R},
$$

while the Spencer operator is given by $\mathrm{D}(\eta, f):=\mathrm{d} f-\eta$, where $(\eta, f) \in \Omega^{1}(P) \oplus C^{\infty}(P)$ (cf. Example 2.16). Moreover, the $T \mathcal{F}$-connection given in Note 3.8 reduces to the Bott connection

$$
\bar{\nabla}: \Gamma(T \mathcal{F}) \times C^{\infty}(P) \rightarrow C^{\infty}(P), \quad(X, f) \mapsto \mathrm{d} f(X) ;
$$

thus the induced differential $\mathrm{d}_{\mathcal{F}}$ is the restriction of the exterior derivative to the foliation $\mathcal{F}$. The cohomology class

$$
\left[\omega_{\mathcal{F}}\right] \in \mathrm{H}^{2}(\mathcal{F} ; L)=\mathrm{H}^{2}(\mathcal{F})
$$

defined by Lemma A.3 is that of the foliated symplectic form defined by $\Lambda$ (cf. Note 5.2). 


\section{Classification of contact isotropic realisations}

As a stepping stone towards classifying CIRs over a fixed Jacobi manifold, some smooth invariants are constructed in analogy with [11]. These are the period lattice and the Chern class. Then we proceed to study in more detail the structure that the period lattice has, giving rise to the notion of transversal $\mathbb{Z}$-projective structures. We finish by providing a cohomological criteria for the existence of CIRs, which combines all the ingredients described before in this section

\subsection{The period lattice and the Chern class}

We first show that any contact realisation of $(P, L,\{\cdot, \cdot\})$ (cf. Definition 3.1) comes equipped with an action of the Lie algebroid $J^{1} L \rightarrow P$. When the realisation is isotropic we called period lattice the isotropy of the action $\Sigma \subset J^{1} L$. Theorem 4.7 shows that in this case, a CIR is in fact locally isomorphic to $\operatorname{Ker} \rho / \Sigma$, hence defining a cohomology class, which we called its Chern class.

Definition 4.1. An action of a Lie algebroid $\mathrm{A} \rightarrow P$ on the smooth manifold $M$ along a map $\phi: M \rightarrow P$ is a vector bundle map $\psi: \phi^{*} \mathrm{~A} \rightarrow T M$ such that

(A1) it induces a Lie algebra homomorphism $\Gamma\left(J^{1} L\right) \rightarrow \mathfrak{X}(M)$;

(A2) for all $m \in M, D_{m} \phi \circ \psi_{m}=\rho_{\phi(m)}$, where $\rho: \mathrm{A} \rightarrow T P$ is the anchor map.

If $\psi$ is injective, the action is said to be faithful.

Lemma 4.2. Let $\phi:(M, H) \rightarrow(P, L,\{\cdot, \cdot\})$ be a contact realisation. The map $\psi: \phi^{*} J^{1} L \rightarrow T M$ given at the level of sections by

$$
\phi^{*} j^{1} u \mapsto R_{\phi^{*} u}, \quad \forall u \in \Gamma(L)
$$

defines a faithful action of $J^{1} L$ on $\phi: M \rightarrow P$.

Note 4.3. Note that $\psi$ can be alternatively described as the restriction of $\rho_{M}$ to $\phi^{*} J^{1} L \subset J^{1} L_{M}$.

Proof of Lemma 4.2. Since $\phi$ is Jacobi, Note 2.13 gives that

$$
D \phi \circ \rho_{M} \circ \phi^{*}=\phi^{*}(\rho) \text {. }
$$

In light of Note 4.3, it follows that property (A2) holds. It remains to prove that $\psi$ induces a Lie algebra morphism, i.e.,

$$
\psi\left(\left[\alpha, \alpha^{\prime}\right]\right)-\left[\psi(\alpha), \psi\left(\alpha^{\prime}\right)\right]=0,
$$

for all $\alpha, \alpha^{\prime} \in \Gamma\left(J^{1} L\right)$. This equation holds when $\alpha, \alpha^{\prime} \in \Gamma\left(J^{1} L\right)$ are holonomic sections, i.e., of the form $j^{1} u, j^{1} v$ for $u, v \in \Gamma(L)$. This is because $\phi$ is a Jacobi map, which implies that

$$
R_{\phi^{*}\{u, v\}}=R_{\left\{\phi^{*} u, \phi^{*} v\right\}}=\left[R_{\phi^{*} u}, R_{\phi^{*} v}\right] .
$$

for $u, v \in \Gamma(L)$, where in the last equality property (2.2) of the Jacobi bracket of $(M, H)$ is used. In general, notice that the left hand side of equation (4.2) satisfies

$$
\psi\left(\left[f \alpha, \alpha^{\prime}\right]\right)-\left[\psi(f \alpha), \psi\left(\alpha^{\prime}\right)\right]=\left(\phi^{*} f\right)\left(\psi\left(\left[\alpha, \alpha^{\prime}\right]\right)-\left[\psi(\alpha), \psi\left(\alpha^{\prime}\right)\right]\right) ;
$$

for $f \in C^{\infty}(P)$ and $\alpha, \alpha^{\prime} \in \Gamma\left(J^{1} L\right)$. This again follows from the fact that $\phi$ is a Jacobi map, so that equation (4.1) holds. Thus, since equation (4.2) holds for holonomic sections and these generate $\Gamma\left(J^{1} L\right)$ as a $C^{\infty}(P)$-module, it follows that equation (4.2) holds for all sections of $J^{1} L$, which proves that $\psi$ satisfies property (A1). The proof of Lemma 3.6 shows that $\psi$ is injective (this follows from property (CR2)). 
Henceforth, let $\phi:(M, H) \rightarrow(P, L,\{\cdot, \cdot\})$ be a CIR unless otherwise stated. Denote the anchor of the Lie algebroid associated to $(P, L,\{\cdot, \cdot\})$ by $\rho: J^{1} L \rightarrow T P$.

Corollary 4.4. The restriction of the action $\psi$ of Lemma 4.2 to the bundle of abelian Lie algebras $\operatorname{ker} \rho \rightarrow P$ defines a faithful action of $\operatorname{ker} \rho \rightarrow P$ on $M$ along $\phi: M \rightarrow P$ which induces a vector bundle isomorphism $\psi: \phi^{*}(\operatorname{ker} \rho) \rightarrow \operatorname{ker} D \phi$.

Proof. The only thing to check is that $\psi: \phi^{*}(\operatorname{ker} \rho) \rightarrow \operatorname{ker} D \phi$ is an isomorphism. Since $\psi$ is injective by Lemma 4.2, it suffices to check that the two vector bundles have the same rank, but this follows from Lemma 3.6.

The action $\psi: \phi^{*} \operatorname{ker} \rho \rightarrow T M$ should be thought of as being infinitesimal; since the fibres of $\phi$ are compact, $\psi$ can be integrated to an action of $\pi$ : $\operatorname{ker} \rho \rightarrow P$ considered as a bundle of abelian Lie groups. The integrated action is given by

$$
\Psi: \quad \operatorname{ker} \rho_{\pi} \times_{\phi} M \rightarrow M, \quad(\alpha, m) \mapsto \varphi_{\alpha}^{1}(m),
$$

where $\operatorname{ker} \rho_{\pi} \times{ }_{\phi} M:=\{(\alpha, m) \in \operatorname{ker} \rho \times M \mid \pi(\alpha)=\phi(m)\}$ is a smooth manifold, and $\varphi_{\alpha}^{1}$ is the time-1 flow of $\psi\left(\phi^{*} \alpha\right)$.

Note 4.5. For each $p \in P$, the action of equation (4.3) restricts to an action of the abelian Lie group ker $\rho_{p} \cong \mathbb{R}^{k}$ on $\phi^{-1}(p)$. Connectivity of $\phi^{-1}(p)$ and Corollary 4.4 imply that the action of ker $\rho_{p}$ is transitive. Moreover, since $\operatorname{ker} \rho_{p}$ is an abelian Lie group, the isotropy of the action at any two points on $\phi^{-1}(p)$ are equal. Therefore, for

$$
\Sigma_{p}:=\left\{\alpha \in \operatorname{ker} \rho_{p} \mid \varphi_{\alpha}^{1}=\mathrm{id}\right\},
$$

the isotropy subgroup at $p$, then

$$
\phi^{-1}(p) \cong \operatorname{ker} \rho_{p} / \Sigma_{p}
$$

since $\phi^{-1}(p)$ is compact by assumption, it follows that $\phi^{-1}(p)$ is diffeomorphic to $\mathbb{T}^{k}$ and that $\Sigma_{p}$ is a cocompact lattice in $\operatorname{ker} \rho_{p}$ and, therefore, isomorphic to $\mathbb{Z}^{k}$.

Just as in the theory of symplectic isotropic realisations of Poisson manifolds (cf. Section 5.1), the isotropy of the action (4.3) plays an important role in the classification of contact isotropic realisations of Jacobi manifolds and endows the foliation of the underlying Jacobi manifold with an interesting transverse geometric structure (cf. Section 4.2).

Definition 4.6. The subset

$$
\Sigma:=\coprod_{p \in P} \Sigma_{p} \subset \operatorname{ker} \rho_{p}
$$

is called the period lattice of the CIR $\phi:(M, H) \rightarrow(P, L,\{\cdot, \cdot\})$.

The following theorem provides a smooth local model for a CIR; its proof is omitted as it is entirely analogous to that of [11, Theorem 2.1].

Theorem 4.7. Let $\phi:(M, H) \rightarrow(P, L,\{\cdot, \cdot\})$ be a CIR with associated period lattice $\Sigma$. Then

(I) $\Sigma$ is a closed submanifold of $\operatorname{ker} \rho$ with the property that the composite $\Sigma \hookrightarrow \operatorname{ker} \rho \rightarrow P$ is $a \mathbb{Z}^{k}$-bundle, i.e., it has fibre $\mathbb{Z}^{k}$ and structure group $\operatorname{GL}(k ; \mathbb{Z})$;

(II) the quotient $\operatorname{ker} \rho / \Sigma$ is a smooth manifold and the projection

$$
\pi: \quad \operatorname{ker} \rho / \Sigma \rightarrow P
$$

is a fibre bundle with fibre $\mathbb{T}^{k}$; 
(III) upon a choice of a local section $\sigma: U \subset M \rightarrow \phi^{-1}(U)$, the map

$$
\Psi_{\sigma}: \quad \operatorname{ker} \rho /\left.\Sigma\right|_{U} \rightarrow \phi^{-1}(U), \quad[\alpha] \mapsto \varphi_{\alpha}^{1}(\sigma(\pi(\alpha)))
$$

is an isomorphism of fibres bundles, making the following diagram

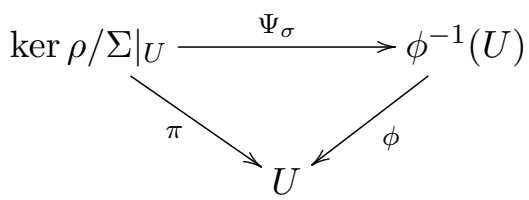

commute;

(IV) the map $\phi: M \rightarrow P$ is a principal ker $\rho / \Sigma$-bundle and is classified up to isomorphism by a cohomology class $t \in \mathrm{H}^{1}\left(P ; \mathcal{C}^{\infty}(\operatorname{ker} \rho / \Sigma)\right)$, where $\mathcal{C}^{\infty}(\operatorname{ker} \rho / \Sigma)$ denotes the sheaf of smooth sections of $\operatorname{ker} \rho / \Sigma \rightarrow P$.

The short exact sequence of sheaves

$$
1 \rightarrow \underline{\Sigma} \hookrightarrow \mathcal{C}^{\infty}(\operatorname{ker} \rho) \rightarrow \mathcal{C}^{\infty}(\operatorname{ker} \rho / \Sigma) \rightarrow 1
$$

where $\underline{\Sigma}$ denotes the sheaf of sections of $\Sigma \rightarrow P$, induces a long exact sequence in cohomology whose connecting morphisms

$$
\delta: \mathrm{H}^{l}\left(P ; \mathcal{C}^{\infty}(\operatorname{ker} \rho / \Sigma)\right) \rightarrow \mathrm{H}^{l+1}(P ; \underline{\Sigma})
$$

are isomorphisms for all $l \geq 1$, since $\mathcal{C}^{\infty}(\operatorname{ker} \rho)$ is fine.

Definition 4.8. The cohomology class

$$
c=\delta(t) \in \mathrm{H}^{2}(P ; \underline{\Sigma})
$$

is called the Chern class of the CIR $\phi:(M, H) \rightarrow(P, L,\{\cdot, \cdot\})$.

\subsection{Period lattices as transversal $\mathbb{Z}$-projective structures}

In order to tackle the problem of determining whether a given regular Jacobi manifold $(P, L,\{\cdot, \cdot\})$ all of whose leaves are even dimensional (see Lemma (3.6)) admits a CIR, it is fundamental to describe necessary conditions for a smooth submanifold $\Sigma \subset$ ker $\rho$ to be the period lattice of some CIR over $(P, L,\{\cdot, \cdot\})$. This is the aim of this subsection, which, in fact, also gives a description of these necessary conditions in terms of a geometric structure transverse to the foliation.

First, local sections of a period lattice of a CIR are characterised in the following lemma, whose statement is analogous to the corresponding condition to be a period lattice of a symplectic isotropic realisation of a Poisson manifold (cf. [11, Corollaire 1]).

Lemma 4.9. If $\Sigma$ is the period lattice of $\phi:(M, H) \rightarrow(P, L,\{\cdot, \cdot\})$ then any local section $\alpha \in \Gamma_{\text {loc }}(\Sigma)$ is of the form $j^{1} u$ for some $u \in \Gamma_{\text {loc }}(L)$.

For expositional reasons, the proof of Lemma 4.9 is postponed until Appendix C. Therefore, the period lattice of a CIR $\phi:(M, H) \rightarrow(P, L,\{\cdot, \cdot\})$ is a full-rank lattice of ker $\rho$ (by Part (I) of Theorem 4.7) whose local sections are holonomic (by Lemma 4.9). Recalling that ker $\rho=$ $\Sigma \otimes_{\mathbb{Z}} \mathbb{R}=: \Sigma^{\mathbb{R}}$ fits in the short exact sequence of equation (3.2), it follows that any period lattice of a CIR is an example of the following object.

Definition 4.10. Let $(N, \mathcal{F})$ be a foliated manifold whose foliation has codimension $l \geq 0$ and fix a line bundle $L \rightarrow N$. A transversal $\mathbb{Z}$-projective lattice on $(N, \mathcal{F})$ is a choice of embedded smooth submanifold $\Sigma \subset J^{1} L$ satisfying: 
(T1) the composite

$$
\Sigma \hookrightarrow J^{1} L \rightarrow N
$$

is a $\mathbb{Z}^{l+1}$-bundle (cf. Part (I) of Theorem 4.7);

(T2) $\Sigma^{\mathbb{R}}:=\Sigma \otimes_{\mathbb{Z}} \mathbb{R}$ fits in a short exact sequence of vector bundles

$$
0 \rightarrow \nu^{*} \otimes L \rightarrow \Sigma^{\mathbb{R}} \rightarrow L \rightarrow 0,
$$

where $\nu^{*} \subset \mathrm{T}^{*} N$ is the conormal bundle to $\mathcal{F}$, and $\Sigma^{\mathbb{R}} \rightarrow L$ is the restriction of the canonical projection $J^{1} L \rightarrow L$;

(T3) any $\alpha \in \Gamma_{\text {loc }}(\Sigma)$ is holonomic.

Example 4.11. If $L=\mathbb{R}_{N}$, then $J^{1} \mathbb{R}_{N}$ is canonically isomorphic to $T^{*} N \oplus \mathbb{R}$. In this case conditions (T1) and (T2) become that $\Sigma$ is a full-rank lattice in $\nu^{*} \oplus \mathbb{R}$, where $\nu^{*}:=(T \mathcal{F})^{\circ} \subset T^{*} N$ is the conormal bundle to $\mathcal{F}$.

The reason for the terminology in Definition 4.10 is that a transversal $\mathbb{Z}$-projective lattice induces a transversal $\mathbb{Z}$-projective structure in the following sense (cf. Proposition 4.14).

Definition 4.12. A transversal $\mathbb{Z}$-projective structure on a foliated manifold $(N, \mathcal{F})$ is an atlas $\mathcal{A}:=\left\{\left(U_{i}, \chi_{i}\right)\right\}$ of submersions $\chi_{i}: U_{i} \rightarrow \mathbb{R} \mathrm{P}^{l}$ locally defining $\mathcal{F}$, such that, for all $i, j$ with $U_{i j}:=U_{i} \cap U_{j} \neq \varnothing$, there exists a smooth map $A_{i j}: U_{i j} \rightarrow \mathrm{GL}(l+1 ; \mathbb{Z})$ satisfying

- $\chi_{j}=\left[A_{i j}\right] \circ \chi_{i}$ on $U_{i j}$, where $\left[A_{i j}\right]: \mathbb{R P}^{l} \rightarrow \mathbb{R P}^{l}$ is the map induced by $A_{i j}$;

- $\left\{A_{i j}\right\}$ satisfies the cocycle condition.

The components of $\chi_{j}$ are called local transversal $\mathbb{Z}$-projective coordinates on $(N, \mathcal{F})$.

Note 4.13. Transversal $\mathbb{Z}$-projective structures are examples of transversal projective structures on foliated manifolds, which can be defined as follows. If $(N, \mathcal{F})$ is a foliated manifold and $l \geq 0$ is the codimension of $\mathcal{F}$, then a transversal projective structure on $(N, \mathcal{F})$ is an atlas $\mathcal{A}:=\left\{\left(U_{i}, \chi_{i}\right)\right\}$ of submersions $\chi_{i}: U_{i} \rightarrow \mathbb{R P}^{l}$ locally defining $\mathcal{F}$, such that, for all $i, j$ with $U_{i j}:=U_{i} \cap U_{j} \neq \varnothing$, there exists a smooth map $\mathrm{a}_{i j}: U_{i j} \rightarrow \operatorname{PGL}(l+1 ; \mathbb{R})$ satisfying $\chi_{j}=\mathrm{a}_{i j} \circ \chi_{i}$ on $U_{i j}$.

Proposition 4.14. There is a 1-1 correspondence between transversal $\mathbb{Z}$-projective lattices and transversal $\mathbb{Z}$-projective structures on a foliated manifold $(N, \mathcal{F})$. In this $1-1$ correspondence, locally

$$
\Sigma:=\mathbb{Z}\left\langle j^{1} x_{1}, \ldots, j^{1} x_{l+1}\right\rangle,
$$

where $\left[x_{1}: \ldots: x_{l+1}\right]$ are local transversal $\mathbb{Z}$-projective coordinates.

The proof of Proposition 4.14 is given in Appendix C.

It is instructive to see the above correspondence in a specific example, which can be considered to be 'universal'.

Example 4.15 (a $\mathbb{Z}$-projective lattice and structure on $\mathbb{R P}^{l}$ ). Fix $l \geq 0$ and consider the line bundle $\pi: O(1) \rightarrow \mathbb{R P}^{l}$. The standard coordinates $x_{1}, \ldots, x_{l+1}$ on $\mathbb{R}^{l+1}$ define global sections of $O(1)$, whose first jets are independent everywhere. In other words, $J^{1}(O(1)) \cong$ $\mathbb{R}\left\langle j^{1} x_{1}, \ldots, j^{1} x_{l+1}\right\rangle$. Define

$$
\Sigma^{l}:=\mathbb{Z}\left\langle j^{1} x_{1}, \ldots, j^{1} x_{l+1}\right\rangle \subset J^{1}(O(1)) ;
$$


it can be verified directly that $\Sigma^{l}$ defines a $\mathbb{Z}$-projective lattice on $\mathbb{R} \mathrm{P}^{l}$ with respect to the foliation by points ${ }^{3}$. On the other hand, there is a canonical $\mathbb{Z}$-projective structure on $\mathbb{R P}^{l}$, constructed by taking the identity $\mathbb{R P}^{l} \rightarrow \mathbb{R P}^{l}$ as the coordinate map and the maps $A_{i j} \in$ $\mathrm{GL}(l+1 ; \mathbb{Z})$ also equal to the identity. The above lattice and structure are mapped one to the other under the correspondence of Proposition 4.14.

\subsection{The realisation problem for CIRs}

The aim of this section is to solve the realisability problem for CIRs, outlined below. Given a regular Jacobi manifold $(P, L,\{\cdot, \cdot\})$ all of whose leaves are even dimensional, let $\mathcal{F}$ denote the induced foliation on $P$, and fix a transversal $\mathbb{Z}$-projective lattice $\Sigma \subset \operatorname{ker} \rho$, where $\rho$ is the anchor map of $J^{1} L$ (cf. Definition 4.10). Natural questions to address are

- to determine which cohomology classes in $\mathrm{H}^{2}(P ; \Sigma)$ correspond to Chern classes of CIR of $(P, L,\{\cdot, \cdot\})$ whose period lattice is $\Sigma$, and

- supposing that $c \in \mathrm{H}^{2}(P ; \Sigma)$ is the Chern class of a CIR with period lattice $\Sigma$, to determine all the CIRs with period lattice $\Sigma$ and Chern class $c$ up to the following notion of isomorphism.

Definition 4.16. Two CIRs $\phi:(M, H) \rightarrow(P, L,\{\cdot, \cdot\})$ and $\phi^{\prime}:\left(M^{\prime}, H^{\prime}\right) \rightarrow(P, L,\{\cdot, \cdot\})$ are said to be isomorphic if there exists a diffeomorphism $I: M \rightarrow M^{\prime}$ satisfying $\phi^{\prime} \circ I=\phi$ and $I^{*} \theta^{\prime}=\theta$, where $\theta$ and $\theta^{\prime}$ are the generalised contact forms associated to $H$ and $H^{\prime}$ respectively.

Remark 4.17. Definition 4.16 provides a notion of strict isomorphism of CIRs, i.e., the bundle component of the Jacobi morphism $I:(M, H) \rightarrow\left(M^{\prime}, H^{\prime}\right)$ is the identity. It is possible to define a more general notion of isomorphism of CIRs which allows for arbitrary bundle components, and to prove a result analogous to Part (2) of Theorem 4.18 for this more general notion of isomorphism using the ideas presented below. For simplicity, only the case of strict isomorphism is considered here.

The main result of this section, Theorem 4.18, provides cohomological criteria that solve the above problems. Recall that, associated to a Jacobi manifold $(P, L,\{\cdot, \cdot\})$ as above, there is a canonical foliated, closed 2 -form $\omega_{\mathcal{F}}$ with values in $L$ (cf. Note 3.8). On the other hand, it can be shown that the Spencer operator D: $\Gamma\left(J^{1} L\right) \rightarrow \Omega^{1}(P ; L)$ associated to $(P, L,\{\cdot, \cdot\})$ induces maps in cohomology

$$
\mathcal{D}: \mathrm{H}^{l}\left(P ; \mathcal{C}^{\infty}(\operatorname{ker} \rho / \Sigma)\right) \rightarrow \mathrm{H}^{l}\left(P, \mathcal{Z}^{1}(\mathcal{F} ; L)\right)
$$

for all $l \geq 0$ (cf. the discussion following Corollary 4.19). For $l \geq 1$, there are isomorphisms $\mathrm{H}^{l}\left(P ; \mathcal{C}^{\infty}(\operatorname{ker} \rho / \Sigma)\right) \cong \mathrm{H}^{l+1}(P ; \underline{\Sigma})(\operatorname{cf}$. Section 4$)$, and $\mathrm{H}^{l}\left(P, \mathcal{Z}^{1}(\mathcal{F} ; L)\right) \cong \mathrm{H}^{l+1}(\mathcal{F} ; L)$ via a standard double Cech-Lie algebroid differential complex argument. Hence, for all $l \geq 1$, the homomorphism of equation (4.5) induces a homomorphism

$$
\mathrm{H}^{l+1}(P ; \underline{\Sigma}) \rightarrow \mathrm{H}^{l+1}(\mathcal{F} ; L),
$$

which, by abuse of notation, is also denoted by $\mathcal{D}$.

\section{Theorem 4.18.}

1. A Jacobi manifold $(P, L,\{\}$,$) admits a CIR with period lattice \Sigma$ and Chern class $c \in$ $\mathrm{H}^{2}(P ; \Sigma)$ if and only if

$$
\mathcal{D} c=\left[\omega_{\mathcal{F}}\right] .
$$

\footnotetext{
${ }^{3}$ Whenever the foliation is by points, the adjective 'transversal' is omitted.
} 
2. The CIRs over $(P, L,\{\cdot, \cdot\})$ with period lattice $\Sigma$ and Chern class $c$ are classified up to isomorphism by $\mathrm{H}^{1}(\mathcal{F} ; L)$. The correspondence is given as follows: if $\phi:(M, H) \rightarrow$ $(P, L,\{\cdot, \cdot\})$ is such a CIR, $\theta$ is the generalised contact form defining $H$, and $\left[\eta_{\mathcal{F}}\right] \in$ $\mathrm{H}^{1}(\mathcal{F} ; L)$, then any other $C I R$ over $(P, L,\{\cdot, \cdot\})$ with period lattice $\Sigma$ and Chern class $c$ is isomorphic to

$$
\phi:\left(M, H^{\prime}=\operatorname{ker}\left(\theta+\phi^{*} \eta\right)\right) \rightarrow(P, L,\{\cdot, \cdot\}),
$$

where $\eta \in \Omega^{1}(P ; L)$ is a 1 -form which represents $\left[\eta_{\mathcal{F}}\right]$.

An immediate corollary of Theorem 4.18 is the classification of CIR for the zero Jacobi bracket on the trivial line bundle, obtained in [1, 25, 32] (the last two deal with singularities coming from the existence of a global contact toric action). Given a manifold $P$, let $\left(P, 0, \mathbb{R}_{P}\right)$ denote the Jacobi manifold corresponding to the zero Jacobi structure on the trivial line bundle.

Corollary 4.19. Given any $\mathbb{Z}$-projective lattice $\Sigma \subset J^{1} P$ on the Jacobi manifold $\left(P, 0, \mathbb{R}_{P}\right)$, any cohomology class $c \in \mathrm{H}^{2}(P ; \underline{\Sigma})$ corresponds to a unique $C I R$ of $\left(P, 0, \mathbb{R}_{P}\right)$ with period lattice $\Sigma$ up to isomorphism.

Proof. Fix $\left(P, 0, \mathbb{R}_{P}\right)$ and a $\mathbb{Z}$-projective lattice $\Sigma \subset J^{1} P=\operatorname{ker} \rho, \rho: J^{1} P \rightarrow T P$ being the anchor map (which is identically zero in this case!). The induced foliation $\mathcal{F}$ is by points and, therefore, the sheaf $\mathcal{Z}^{1}(\mathcal{F}):=\mathcal{Z}^{1}(\mathcal{F} ; \mathbb{R})=\Omega^{1}(P)$ is fine, thus showing, in particular, that $\mathrm{H}^{2}(\mathcal{F})=0$. Therefore the homomorphism $\mathcal{D}: \mathrm{H}^{2}(P ; \underline{\Sigma}) \rightarrow \mathrm{H}^{2}(\mathcal{F})$ is trivial and every cohomology class can be realised as the Chern class of some CIR of $\left(P, 0, \mathbb{R}_{P}\right)$ with period lattice $\Sigma$. To prove uniqueness up to isomorphism, observe that, since the foliation is by points, every closed foliated 1 -form is exact.

The rest of this subsection provides the necessary preparatory material as well as the proof of Theorem 4.18, which is at the end of the subsection. Some of the intermediate results are interesting on their own; for instance, Theorem 4.22 gives a local normal form for the contact structure of a CIR, which generalises [1, Theorem 1] and [21, Theorems 4 and 5]. Henceforth, fix a regular Jacobi manifold $(P, L,\{\cdot, \cdot\})$ all of whose leaves are even dimensional, let $\mathcal{F}$ denote the induced foliation on $P$, and fix a transversal $\mathbb{Z}$-projective lattice $\Sigma \subset \operatorname{ker} \rho$, where $\rho: J^{1} L \rightarrow T P$ is the anchor map. First, the homomorphism $\mathcal{D}$ is constructed starting from the Spencer operator D: $\Gamma\left(J^{1} L\right) \rightarrow \Omega^{1}(M ; L)$ associated to the Lie algebroid $J^{1} L \rightarrow P$ (cf. Note 2.17 and the ensuing discussion). Recall that there is a foliated flat connection $\bar{\nabla}$ on $L$ uniquely defined by equation (3.4) (cf. Note 3.8 and Lemma A.1).

Note 4.20. For any $\alpha \in \Gamma(\operatorname{ker} \rho), \mathrm{d}_{\mathcal{F}} \mathrm{D}(\alpha)=0$; in fact $\mathrm{D}(\alpha)=\mathrm{d}_{\mathcal{F}}(\operatorname{pr}(\alpha))$, where pr: $\operatorname{ker} \rho \rightarrow L$ is the projection (cf. Note 3.8 and equation (3.2)). The proof of this result can be found in Appendix A.

By Note 4.20, the Spencer operator induces a sheaf homomorphism $\overline{\mathrm{D}}: \mathcal{C}^{\infty}(\operatorname{ker} \rho) \rightarrow \mathcal{Z}^{1}(\mathcal{F} ; L)$, where $\mathcal{Z}^{1}(\mathcal{F} ; L)$ denotes the sheaf of closed, foliated forms with values in $L$. If $\mathcal{C}^{\infty}(\operatorname{ker} \rho / \Sigma)$ denotes the sheaf of smooth sections of $\operatorname{ker} \rho / \Sigma \rightarrow P$, Lemma 4.9 implies that there is a morphism of sheaves

$$
\hat{\mathrm{D}}: \mathcal{C}^{\infty}(\operatorname{ker} \rho / \Sigma) \rightarrow \mathcal{Z}^{1}(\mathcal{F} ; L),
$$

which induces the homomorphisms at the level of cohomology of equation (4.5). The strategy of the proof of Theorem 4.18 hinges upon a strengthened version of Theorem 4.7, i.e., on a contact local normal form for sufficiently small open $\phi$-saturated neighbourhoods. One of the main ingredients of this result is the following. 
Note 4.21. Consider the canonical contact form $\theta_{\text {can }} \in \Omega^{1}\left(J^{1} L ; \mathrm{pr}^{*} L\right)$ described in Example 2.4. Translations by elements of $\Sigma$, which are holonomic by Lemma 4.9, preserve $\theta_{\text {can }}$ (cf. Note 2.17), and therefore its restriction to $\operatorname{ker} \rho \subset J^{1} L$ descends to a 1 -form

$$
\theta_{0} \in \Omega^{1}\left(\operatorname{ker} \rho / \Sigma ; \pi^{*} L\right),
$$

which does not necessarily define a contact distribution (unless ker $\rho=J^{1} L$ ).

Fix a CIR $\phi:(M, H) \rightarrow(P, L,\{\}$,$) and let \theta \in \Omega^{1}\left(M, L_{M}\right)$ denote the generalised contact form associated to $(M, H)$.

Theorem 4.22. Given a local section $\sigma: U \subset P \rightarrow(M, H)$, then

$$
\Psi_{\sigma}^{*} \theta=\theta_{0}+\pi^{*} \sigma^{*} \theta
$$

where $\Psi_{\sigma}$ : $\operatorname{ker} \rho /\left.\Sigma\right|_{U} \rightarrow \phi^{-1}(U)$ is defined by equation (4.4). Moreover, $\mathrm{d}_{\mathcal{F}}\left(\sigma^{*} \theta\right)=\omega_{\mathcal{F}}$.

The proof of Theorem 4.22 is postponed to Appendix C. The following result (stated without proof as it can be checked using local coordinates) provides a partial converse to Theorem 4.22.

Proposition 4.23. Let $U \subset P$ be an open set and fix $\beta \in \Omega^{1}(U, L)$ satisfying $\mathrm{d}_{\mathcal{F}} \beta=\omega_{\mathcal{F}}$. Then

$$
\pi:\left(\operatorname{ker} \rho /\left.\Sigma\right|_{U}, \theta_{0}+\pi^{*} \beta\right) \rightarrow\left(U, L_{U},\{\cdot, \cdot\}\right)
$$

is a contact isotropic realisation.

Finally, it is possible to proceed with the proof of the main result of this section.

Proof of Theorem 4.18. The strategy is similar to that of [11, Theorems 4.2 and 4.3]. Throughout the proof, let $\mathcal{U}:=\left\{U_{i}\right\}$ denote a good open cover of $P$.

Part (1). Suppose first that $\phi:(M, H) \rightarrow(P, L,\{\cdot, \cdot\})$ is a CIR with Chern class $c \in$ $\mathrm{H}^{2}(P ; \Sigma)$, and let $\theta$ be the generalised contact form associated to $(M, H)$. For each $i$, let $\sigma_{i}: U_{i} \rightarrow M$ be a local section of $\phi$; as in Theorem 4.7, the section $\sigma_{i}$ induces a local trivialisation $\Psi_{i}$ : $\operatorname{ker} \rho /\left.\Sigma\right|_{U_{i}} \rightarrow \phi^{-1}\left(U_{i}\right)$. By Definition 4.8, a Čech cocycle representing $c$ is given by the smooth maps $t_{i j}: U_{i j} \rightarrow \operatorname{ker} \rho / \Sigma$ defined by

$$
\Psi_{j}^{-1} \circ \Psi_{i}([\alpha])=[\alpha]+t_{i j}(\pi(\alpha))
$$

where $\pi: \operatorname{ker} \rho / \Sigma \rightarrow P$ is the projection (cf. the discussion following Theorem 4.7). Thus a Čech cocycle representing $\mathcal{D} c$ is given by $\left\{t_{i j}^{*} \theta_{0}=\hat{\mathrm{D}}\left(t_{i j}\right)\right\}-$ cf. Note 4.21. By definition of $t_{i j}$, $\Psi_{i} \circ t_{i j}=\sigma_{j}$ on $U_{i j}$; thus

$$
\sigma_{j}^{*} \theta=t_{i j}^{*} \circ \Psi_{i}^{*} \theta=t_{i j}^{*}\left(\theta_{0}+\pi^{*} \sigma_{i}^{*} \theta\right)=t_{i j}^{*} \theta_{0}+\sigma_{i}^{*} \theta,
$$

where the second equality uses Theorem 4.22, and the last follows by noticing that $\pi \circ t_{i j}=\mathrm{id}$. Equation (4.7) gives that $t_{i j}^{*} \theta_{0}=\sigma_{j}^{*} \theta-\sigma_{i}^{*} \theta$. By Theorem 4.22, $\mathrm{d}_{\mathcal{F}}\left(\sigma_{j}^{*} \theta\right)=\omega=\mathrm{d}_{\mathcal{F}}\left(\sigma_{i}^{*} \theta\right)$; this implies that the cohomology class of $\left\{\sigma_{j}^{*} \theta-\sigma_{i}^{*} \theta\right\}$ equals $\left[\omega_{\mathcal{F}}\right]$, as it is the difference of two primitives of $\omega_{\mathcal{F}}$.

Conversely, suppose that $\mathcal{D} c=\left[\omega_{\mathcal{F}}\right]$. Choose the good open cover $\mathcal{U}$ so that, for each $i$, there exists $\beta_{i} \in \Omega^{1}\left(\left.\mathcal{F}\right|_{U_{i}} ; L\right)$ with $\mathrm{d}_{\mathcal{F}} \beta_{i}=\left.\omega_{\mathcal{F}}\right|_{U_{i}}$. Since $\mathcal{F}$ is regular, the foliated 1-forms $\beta_{i}$ can be extended to elements of $\Omega^{1}\left(U_{i} ; L\right)$, which are henceforth also denoted by $\beta_{i}$ by abuse of notation. Since $\mathcal{D} c=\left[\omega_{\mathcal{F}}\right]$, there exists a Čech cocycle $\left\{t_{i j}\right\}$ representing $c$ which satisfies $t_{i j}^{*} \theta_{0}=\beta_{j}-\beta_{i}$ on $U_{i j}$. For each $i$, consider the CIR $\pi:\left(\operatorname{ker} \rho /\left.\Sigma\right|_{U_{i}}, \theta_{0}+\pi^{*} \beta_{i}\right) \rightarrow\left(U_{i},\left.L\right|_{U_{i}},\left.\{\cdot, \cdot\}\right|_{U_{i}}\right)$ (cf. Proposition 4.23). The principal ker $\rho / \Sigma$-bundle over $P$ with Chern class $c$ is constructed (up to isomorphism) by glueing the above local models using the translations $t_{i j}$. The condition 
$t_{i j}^{*} \theta_{0}=\beta_{j}-\beta_{i}$ ensures that the locally defined generalised contact forms $\theta_{0}+\pi^{*} \beta_{i}$ patch together to give a globally defined generalised contact form $\theta \in \Omega^{1}\left(M ; \phi^{*} L\right)$, where $\phi: M \rightarrow P$ is the principal $\operatorname{ker} \rho / \Sigma$-bundle over $P$ with Chern class $c$ constructed above. Setting $H:=\operatorname{ker} \theta$, have that $\phi:(M, H) \rightarrow(P, L,\{\cdot, \cdot\})$ is a CIR as it suffices to check that the properties (CR1), (CR2) and (I) hold locally and they do by construction, since the bundle is obtained by glueing CIR.

Part (2). Suppose that $\mathcal{D} c=\left[\omega_{\mathcal{F}}\right]$; by Part (1), there exists a CIR $\phi:(M, H) \rightarrow(P, L,\{\cdot, \cdot\})$ with period lattice $\Sigma$ and Chern class $c$, which is henceforth fixed as a 'reference'. Suppose that $\phi^{\prime}:\left(M^{\prime}, H^{\prime}\right) \rightarrow(P, L,\{\cdot, \cdot\})$ is another CIR with period lattice $\Sigma$ and Chern class $c$. By composing with an isomorphism of principal ker $\rho / \Sigma$-bundles, it may be assumed that $\phi^{\prime}=\phi$ and that $M^{\prime}=M$. Denote the generalised contact forms associated to $H$ and $H^{\prime}$ by $\theta$ and $\theta^{\prime}$ respectively. For each $i$, consider the form $\eta_{i}:=\sigma_{i}^{*}\left(\theta-\theta^{\prime}\right)$. By Theorem $4.22, \mathrm{~d}_{\mathcal{F}} \eta_{i}=0$. Moreover, on $U_{i j}$,

$$
\eta_{i}-\eta_{j}=\sigma_{i}^{*}\left(\theta-\theta^{\prime}\right)-\sigma_{j}^{*}\left(\theta-\theta^{\prime}\right)=\left(\sigma_{i}^{*} \theta-\sigma_{j}^{*} \theta\right)-\left(\sigma_{i}^{*} \theta^{\prime}-\sigma_{j}^{*} \theta^{\prime}\right)=t_{i j}^{*} \theta_{0}-t_{i j}^{*} \theta_{0}=0 ;
$$

thus the collection $\left\{\eta_{i}\right\}$ defines a globally defined foliated closed 1-form $\eta_{\mathcal{F}}=\eta_{\mathcal{F}}\left(\theta, \theta^{\prime}\right) \in$ $\mathrm{H}^{0}\left(P ; \mathcal{Z}^{1}(\mathcal{F} ; L)\right)=\mathrm{Z}^{1}(\mathcal{F} ; L)$. In other words, there is a function (depending on the reference $\operatorname{CIR} \phi:(M, H) \rightarrow(P, L,\{\cdot, \cdot\}) !)$

$b_{\theta}:\{$ CIRs with period lattice $\Sigma$ and Chern class $c\} \rightarrow \mathrm{Z}^{1}(\mathcal{F} ; L)$,

$$
\left(\phi^{\prime}:\left(M^{\prime}, H^{\prime}\right) \rightarrow(P, L,\{\cdot, \cdot\})\right) \mapsto \eta_{\mathcal{F}}\left(\theta, \theta^{\prime}\right),
$$

where $\mathrm{Z}^{1}(\mathcal{F} ; L)$ denotes the vector space of globally defined closed, foliated 1-forms with values in $L$. Next it is shown that $b_{\theta}$ is surjective. Let $\eta_{\mathcal{F}} \in \mathrm{Z}^{1}(\mathcal{F} ; L)$ and let $\eta \in \Omega^{1}(P ; L)$ be any 1 -form extending it; then Proposition 4.23 ensures that $\phi:\left(M, H^{\prime}\right) \rightarrow(P, L,\{\cdot, \cdot\})$ is a CIR with period lattice $\Sigma$ and Chern class $c$, where $H^{\prime}=\operatorname{ker}\left(\theta+\phi^{*} \eta\right)$. Setting $\theta^{\prime}=\theta+\phi^{*} \eta$, it is simple to check that $\eta_{\mathcal{F}}\left(\theta, \theta^{\prime}\right)=\eta_{\mathcal{F}}$, thus showing that $b_{\theta}$ is onto. However, $b_{\theta}$ does not descend to a function on the set of isomorphism classes of CIRs with period lattice $\Sigma$ and Chern class $c$. For, if $\phi:(M, H) \rightarrow(P, L,\{\cdot, \cdot\})$ and $\phi:\left(M, H^{\prime}\right) \rightarrow(P, L,\{\cdot, \cdot\})$ are isomorphic in the sense of Definition 4.16, then $\eta_{\mathcal{F}}\left(\theta, \theta^{\prime}\right) \in \hat{\mathrm{D}}(\Gamma(\operatorname{ker} \rho / \Sigma))$; this can be proved as follows. Using Theorem 4.22, for each $i$ there exists an isomorphism of CIRs

$$
T_{i}:\left(\operatorname{ker} \rho /\left.\Sigma\right|_{U_{i}}, \theta_{0}+\pi^{*} \sigma_{i}^{*} \theta\right) \rightarrow\left(\operatorname{ker} \rho /\left.\Sigma\right|_{U_{i}}, \theta_{0}+\pi^{*} \sigma_{i}^{*} \theta^{\prime}\right) .
$$

Fix $i$. Since an isomorphism of CIRs is, in particular, an isomorphism of principal ker $\rho / \Sigma$ bundles, it follows that there exists a section $\tau_{i} \in \Gamma\left(\operatorname{ker} \rho /\left.\Sigma\right|_{U_{i}}\right)$ such that $T_{i}(\alpha+\Sigma)=\alpha+\tau_{i}+\Sigma$. Using the fact that $T_{i}^{*}\left(\theta_{0}+\pi^{*} \sigma_{i}^{*} \theta^{\prime}\right)=\theta_{0}+\pi^{*} \sigma_{i}^{*} \theta^{\prime}$, i.e., $T_{i}$ is an isomorphism of CIRs, it follows that $\hat{\mathrm{D}}\left(\tau_{i}\right)=\eta_{i}$, as $\tau_{i}^{*} \theta_{0}=\hat{\mathrm{D}}\left(\tau_{i}\right)$. This holds for all $i$; however, since the collection of isomorphisms $\left\{T_{i}\right\}$ patches together to give a global isomorphism of CIRs, it follows that for all $i, j$ with $U_{i j} \neq \varnothing, T_{j} \circ t_{i j}=t_{i j} \circ T_{i}$, which is equivalent to $\tau_{i}=\tau_{j}$ on $U_{i j}$. This shows that the collection $\left\{\tau_{i}\right\}$ defines a globally defined section $\tau \in \mathrm{H}^{0}\left(P ; \mathcal{C}^{\infty}(\operatorname{ker} \rho / \Sigma)\right)=\Gamma(\operatorname{ker} \rho / \Sigma)$; since, for all $i$, $\hat{\mathrm{D}}\left(\tau_{i}\right)=\eta_{i}$, it follows that the 1-form $\eta_{\mathcal{F}}\left(\theta, \theta^{\prime}\right) \in \hat{\mathrm{D}}(\Gamma(\operatorname{ker} \rho / \Sigma))$. In fact, something more general is true: if $\phi:\left(M, H^{\prime}\right) \rightarrow(P, L,\{\cdot, \cdot\})$ and $\phi:\left(M, H^{\prime \prime}\right) \rightarrow(P, L,\{\cdot, \cdot\})$ are isomorphic CIRs, then $\eta_{\mathcal{F}}\left(\theta, \theta^{\prime}\right)-\eta_{\mathcal{F}}\left(\theta, \theta^{\prime \prime}\right) \in \hat{\mathrm{D}}(\Gamma($ ker $\rho / \Sigma))$, where $\theta, \theta^{\prime}, \theta^{\prime \prime}$ are the generalised contact forms associated to $H, H^{\prime}$ and $H^{\prime \prime}$ respectively. This follows from the fact that

$$
\eta_{\mathcal{F}}\left(\theta^{\prime}, \theta^{\prime \prime}\right)=\eta_{\mathcal{F}}\left(\theta, \theta^{\prime \prime}\right)-\eta_{\mathcal{F}}\left(\theta, \theta^{\prime}\right)
$$

which is a direct consequence of the definition of the forms $\eta_{\mathcal{F}}\left(\theta^{\prime}, \theta^{\prime \prime}\right), \eta_{\mathcal{F}}\left(\theta, \theta^{\prime \prime}\right)$, and $\eta_{\mathcal{F}}\left(\theta, \theta^{\prime}\right)-$ to define $\eta_{\mathcal{F}}\left(\theta^{\prime}, \theta^{\prime \prime}\right), \phi:\left(M, H^{\prime}\right) \rightarrow(P, L,\{\cdot, \cdot\})$ is taken to be the 'reference' CIR. The above argument proves that $\eta_{\mathcal{F}}\left(\theta^{\prime}, \theta^{\prime \prime}\right) \in \hat{\mathrm{D}}(\Gamma(\operatorname{ker} \rho / \Sigma))$, which yields the claimed result. Moreover, if $\eta_{\mathcal{F}}=\hat{\mathrm{D}} \tau$ for some $\tau \in \Gamma(\operatorname{ker} \rho / \Sigma)$, reverse the above reasoning to obtain that the CIRs 
$\phi:(M, H) \rightarrow(P, L,\{\cdot, \cdot\})$ and $\phi:\left(M, H^{\prime}\right) \rightarrow(P, L,\{\cdot, \cdot\})$ are isomorphic, where $H^{\prime}=\operatorname{ker}(\theta+$ $\phi^{*} \eta$ ), where $\eta$ is a 1 -form extending $\eta_{\mathcal{F}}$. In conclusion, the above discussion implies that there exists a well-defined bijection

$$
\begin{aligned}
& \frac{\{\text { CIRs with period lattice } \Sigma \text { and Chern class } c\}}{\sim} \rightarrow \frac{\mathrm{Z}^{1}(\mathcal{F} ; L)}{\hat{\mathrm{D}}(\Gamma(\operatorname{ker} \rho / \Sigma))}, \\
& {\left[\phi^{\prime}:\left(M^{\prime}, H^{\prime}\right) \rightarrow(P, L,\{\cdot, \cdot\})\right] \mapsto \eta\left(\theta, \theta^{\prime}\right)+\hat{\mathrm{D}}(\Gamma(\operatorname{ker} \rho / \Sigma)),}
\end{aligned}
$$

where $\sim$ denotes the equivalence relation defined by the notion of isomorphism of Definition 4.16. To complete the proof, it suffices to prove that $\hat{\mathrm{D}}(\Gamma(\operatorname{ker} \rho / \Sigma))$ is precisely the vector space of exact, globally defined foliated 1-forms with values in $L$. By Note 4.20, if $\alpha+\Sigma \in \Gamma(\operatorname{ker} \rho / \Sigma)$, then $\hat{\mathrm{D}}(\alpha+\Sigma)=\mathrm{d}_{\mathcal{F}}(\operatorname{pr}(\alpha))$. Therefore, $\hat{\mathrm{D}}(\Gamma(\operatorname{ker} \rho / \Sigma))$ consists of exact, foliated 1-forms with values in $L$. Suppose that $\mathrm{d}_{\mathcal{F}} u$ is an exact foliated 1 -form with values in $L$, where $u \in \Gamma(L)$. Since pr: $\operatorname{ker} \rho \rightarrow L$ is onto (cf. Note 3.8), it follows that there exists a section $\alpha \in \Gamma(\operatorname{ker} \rho)$ with $u=\operatorname{pr}(\alpha)$. Then $\hat{\mathrm{D}}(\alpha+\Sigma)=\mathrm{d}_{\mathcal{F}} u$ by construction, which shows that all exact, foliated 1 -forms with values in $L$ are contained in $\hat{\mathrm{D}}(\Gamma(\operatorname{ker} \rho / \Sigma))$. Hence,

$$
\frac{\mathrm{Z}^{1}(\mathcal{F} ; L)}{\hat{\mathrm{D}}(\Gamma(\operatorname{ker} \rho / \Sigma))}=\mathrm{H}^{1}(\mathcal{F} ; L),
$$

therefore completing the proof.

\section{The case of Poisson manifolds: comparing symplectic and contact isotropic realisations}

\subsection{Symplectic isotropic realisations of Poisson manifolds: a reminder}

For completeness, the theory of symplectic isotropic realisations of Poisson manifolds is recalled here (cf. [11] for further details and proofs of all results stated below).

Definition 5.1. A symplectic realisation of a Poisson manifold $(P, \Lambda)$ is a symplectic manifold $(S, \omega)$ together with a surjective submersion $\Phi:(S, \omega) \rightarrow(P, \Lambda)$ which is a Poisson morphism. If, in addition, the fibres of $\Phi$ are isotropic submanifolds of $(S, \omega)$, the realisation is said to be isotropic.

Symplectic realisations play an important role in the study of Poisson manifolds (cf. [3]); while symplectic isotropic realisations (SIR for short) appear naturally when considering Poisson manifolds of compact types (cf. [5, 6]). Henceforth, all SIRs considered have compact fibres unless otherwise stated.

It is well-known that a Poisson manifold $(P, \Lambda)$ admits a SIR only if it is regular (cf. [11] and compare with Lemma 3.6).

Note 5.2. There are two important cohomology classes that can be attached to a regular Poisson manifold $(P, \Lambda)$ with symplectic foliation $\mathcal{F}$. On the one hand, the Poisson bivector $\Lambda$ induces a foliated cohomology class $\left[\omega_{\mathcal{F}}\right] \in \mathrm{H}^{2}(\mathcal{F})$ (cf. Example 3.9). On the other, if $\omega \in \Omega^{2}(P)$ is a 2form $\omega \in \Omega^{2}(P)$ extending $\omega_{\mathcal{F}}, \mathrm{d} \omega$ is a closed 3 -form whose restriction to the leaves of $\mathcal{F}$ is zero; therefore, it defines a cohomology class $[\mathrm{d} \omega] \in \mathrm{H}_{\text {rel }}^{3}(P ; \mathcal{F})$, where $\mathrm{H}_{\text {rel }}^{*}(P ; \mathcal{F})$ is the cohomology of the subcomplex of forms that vanish along $\mathcal{F}$ (this is sometimes referred to as the cohomology of $P$ relative to $\mathcal{F}$ ). This cohomology class is independent of the choice of $\omega$; the class $v:=[\mathrm{d} \omega]$ is known as the characteristic class of $(P, \Lambda)$.

Fix a $\operatorname{SIR} \Phi:(S, \omega) \rightarrow(P, \Lambda)$, let $\mathcal{F}$ and $\nu^{*}$ denote the regular symplectic foliation on $(P, \Lambda)$ and its conormal bundle respectively. The given SIR induces a full-rank lattice $\Xi \subset \nu^{*}$ with the following properties: 
- $\Phi:(S, \omega) \rightarrow(P, \Lambda)$ is a principal $\nu^{*} / \Xi$-bundle, which is classified by an element (called Chern class $)$ in $\mathrm{H}^{1}\left(P ; \mathcal{C}^{\infty}\left(\nu^{*} / \Xi\right)\right) \cong \mathrm{H}^{2}(P ; \Xi)$, where $\mathcal{C}^{\infty}\left(\nu^{*} / \Xi\right)$ and $\Xi$ denote the sheaves of sections of $\nu^{*} / \Xi \rightarrow P$ and of $\Xi \rightarrow P$ respectively (cf. [11, Corollary 2]);

- any locally defined section of $\Xi \rightarrow P$ is a closed 1-form (cf. [11, Corollary 1]).

The full-rank lattice $\Xi \subset \nu^{*}$ encodes an important geometric structure transverse to the foliation $\mathcal{F}$.

Definition 5.3. Let $(N, \mathcal{F})$ be a foliated manifold, where the codimension of the foliation is $l \geq 0$. A transversal $\mathbb{Z}$-affine structure on $(N, \mathcal{F})$ is an atlas $\mathcal{A}:=\left\{\left(U_{i}, \chi_{i}\right)\right\}$ of submersions $\chi_{i}: U_{i} \rightarrow \mathbb{R}^{l}$ locally defining $\mathcal{F}$, such that, for all $i, j$ with $U_{i j}:=U_{i} \cap U_{j} \neq \varnothing$, there exists a smooth map $h_{i j}: \chi_{i}\left(U_{i j}\right) \rightarrow \chi_{j}\left(U_{i j}\right)$ which is (the restriction of) an $\mathbb{Z}$-affine transformation in $\operatorname{Aff}_{\mathbb{Z}}\left(\mathbb{R}^{l}\right):=\operatorname{GL}(l ; \mathbb{Z}) \ltimes \mathbb{R}^{l}$ such that $\left.\chi_{j}\right|_{U_{i j}}=\left.h_{i j} \circ \chi_{i}\right|_{U_{i j}}$.

Note 5.4. Allowing the maps $h_{i j}$ to be (restrictions of) elements in $\operatorname{Aff}\left(\mathbb{R}^{l}\right):=\operatorname{GL}(l ; \mathbb{R}) \ltimes \mathbb{R}^{l}$, obtain the notion of transversal affine structure on a foliated manifold, which stands to its integral counterpart as transversal projective structures stand to their integral analogues (cf. Definitions 4.12 and 4.13).

The following proposition, stated without proof, establishes the analogue of Proposition 4.14 for transversal $\mathbb{Z}$-affine structures (cf. [6, Proposition 3.2.4]).

Proposition 5.5. If $(N, \mathcal{F})$ is a manifold together with a foliation of codimension $l$, there is a 1-1 correspondence between

- transversal $\mathbb{Z}$-affine structures on $(N, \mathcal{F})$;

- full-rank lattices $\Xi \subset \nu^{*}$ whose local sections are closed.

In this correspondence, $\Xi$ is locally given by

$$
\Xi=\mathbb{Z}\left\langle\mathrm{d} x_{1}, \ldots, \mathrm{d} x_{l}\right\rangle
$$

where $x_{1}, \ldots, x_{l}$ are local transversal $\mathbb{Z}$-affine coordinates on $(N, \mathcal{F})$.

Henceforth, any full-rank lattice corresponding to a transversal $\mathbb{Z}$-affine structure as in Proposition 5.5 is referred to as a transversal $\mathbb{Z}$-affine lattice. The realisation problem for SIRs has been solved in [11]. Suppose that $(P, \Lambda)$ is a regular Poisson manifold endowed with a transversal $\mathbb{Z}$-affine lattice $\Xi \subset \nu^{*}$. Since the sections of $\Xi$ are closed, the standard exterior derivative induces a homomorphism of sheaves

$$
\begin{aligned}
\hat{\mathrm{d}}: & \mathcal{C}^{\infty}\left(\nu^{*} / \Xi\right) \rightarrow \mathcal{Z}^{2}\left(\nu^{*}\right), \\
& \alpha+\Xi \mapsto \mathrm{d} \alpha,
\end{aligned}
$$

where $\mathcal{Z}^{2}\left(\nu^{*}\right)$ denotes the sheaf of closed 2 -forms which vanish when restricted to $\mathcal{F}$. For any $l \geq 0$, this induces homomorphisms in cohomology

$$
\mathfrak{d}: \mathrm{H}^{l}\left(P ; \mathcal{C}^{\infty}\left(\nu^{*} / \Xi\right)\right) \rightarrow \mathrm{H}^{l}\left(P ; \mathcal{Z}^{2}\left(\nu^{*}\right)\right) .
$$

For $l \geq 1$, there exist isomorphisms

$$
\mathrm{H}^{l}\left(P ; \mathcal{C}^{\infty}\left(\nu^{*} / \Xi\right)\right) \cong \mathrm{H}^{l+1}(P ; \Xi)
$$

and

$$
\mathrm{H}^{l}\left(P ; \mathcal{Z}^{2}\left(\nu^{*}\right)\right) \cong \mathrm{H}_{\text {rel }}^{l+2}(P ; \mathcal{F}) .
$$


The former arise from considering the short exact sequence of sheaves

$$
0 \rightarrow \Xi \rightarrow \mathcal{C}^{\infty}\left(\nu^{*}\right) \rightarrow \mathcal{C}^{\infty}\left(\nu^{*} / \Xi\right) \rightarrow 0
$$

and by observing that $\mathcal{C}^{\infty}\left(\nu^{*}\right)$ is fine; the latter are explained in [11, Section 4, Part (a)]. The homomorphisms of equation (5.1) induce, for all $l \geq 1$, homomorphisms $\mathrm{H}^{l+1}(P ; \Xi) \rightarrow$ $\mathrm{H}_{\text {rel }}^{l+2}(P ; \mathcal{F})$, which are also denoted by $\mathfrak{d}$ by abuse of notation. With the above homomorphisms at hand, the following results can be stated (cf. [11, Theorems 4.2 and 4.3] for a proof, and compare with Theorem 4.18).

\section{Theorem 5.6.}

1. A regular Poisson manifold $(P, \Lambda)$ admits a symplectic isotropic realisation with period lattice $\Xi$ and Chern class $c \in \mathrm{H}^{2}(P ; \Xi)$ if and only if

$$
\mathfrak{d} c=v,
$$

where $v \in \mathrm{H}_{\text {rel }}^{3}(P ; \mathcal{F})$ is the characteristic class of $(P, \Lambda)$.

2. Suppose that $c \in \mathrm{H}^{2}(P ; \Xi)$ satisfies equation (5.2), then the set of isomorphism classes of symplectic isotropic realisations of $(P, \Lambda)$ with period lattice $\Xi$ and Chern class $c$ is in bijection with

$$
\frac{\mathrm{H}^{0}\left(P ; \mathcal{Z}^{2}\left(\nu^{*}\right)\right)}{\mathfrak{d}\left(\mathrm{H}^{0}\left(P ; \mathcal{C}^{\infty}\left(\nu^{*} / \Xi\right)\right)\right)}=\frac{\mathrm{Z}^{2}\left(\nu^{*}\right)}{\hat{\mathrm{d}}\left(\Gamma\left(\nu^{*} / \Xi\right)\right)},
$$

where $\mathrm{Z}^{2}\left(\nu^{*}\right)$ denotes the vector space of globally defined closed 2 -forms which vanish along the symplectic foliation $\mathcal{F}$.

Note 5.7. The map $\mathfrak{d}: \mathrm{H}^{2}(P ; \Xi) \rightarrow \mathrm{H}_{\text {rel }}^{3}(P ; \mathcal{F})$ can be constructed alternatively as follows. There is a commutative diagram of short exact sequence of sheaves

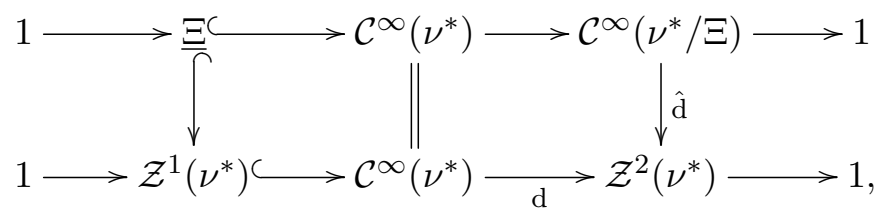

which induces a commutative diagram

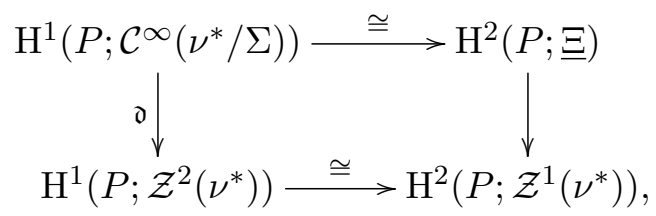

where the vertical maps are induced by the outer vertical maps in equation (5.3) and the horizontal maps are the connecting morphisms in the long exact sequences in cohomology induced by the short exact sequences of equation (5.3), as $\mathcal{C}^{\infty}\left(\nu^{*}\right)$ is a fine sheaf. Under the identifications of equation (5.4), the map $\mathfrak{d}$ can be identified with the homomorphism $\mathrm{H}^{2}(P ; \Xi) \rightarrow \mathrm{H}^{2}\left(P ; \mathcal{Z}^{1}\left(\nu^{*}\right)\right) \cong \mathrm{H}_{\text {rel }}^{3}(P ; \mathcal{F})$ induced by the inclusion $\Xi \hookrightarrow \mathcal{Z}^{1}\left(\nu^{*}\right)$. 


\subsection{Contact isotropic realisations of Poisson manifolds}

It is worth rephrasing some of the results of Section 4.3 in the case in which the underlying Jacobi manifold is, in fact, Poisson, as many of the above objects simplify (cf. Section 5.4 for more applications). Using the notation of Example 2.6, given a Poisson manifold $(P, \Lambda)$, denote the induced Jacobi structure on the trivial bundle $\mathbb{R}_{P}$ also by $(P, \Lambda)$. Suppose that it is regular and denote its symplectic foliation and corresponding conormal bundle by $\mathcal{F}, \nu^{*}$ respectively. Recall that if $\rho: J^{1} P \cong T^{*} P \oplus \mathbb{R} \rightarrow T P$ is the anchor, then ker $\rho=\nu^{*} \oplus \mathbb{R}$ (cf. Example 3.9). Fix a transversal $\mathbb{Z}$-projective lattice $\Sigma \subset \operatorname{ker} \rho$; by definition, any local section of $\Sigma$ is of the form $j^{1} f$, for some $f \in C^{\infty}(P)$. However, since $\Sigma \subset \nu^{*} \oplus \mathbb{R}$,

$$
j^{1} f \in \Gamma(\Sigma) \quad \Rightarrow \quad \mathrm{d} f \in \Gamma\left(\nu^{*}\right) \quad \Leftrightarrow \quad f \text { is a Casimir. }
$$

Remark 5.8. Let $(P, L,\{\cdot, \cdot\})$ be a regular Jacobi manifold all whose leaves are even dimensional. Say that a (locally defined) section $u \in \Gamma(L)$ is a Casimir, if, for all $v \in \Gamma(L),\{u, v\}=0$. Then, using the fact that the structure is regular and all its leaves are even dimensional, it can be shown that $u \in \Gamma(L)$ is a Casimir if and only if $j^{1} u \in \Gamma(\operatorname{ker} \rho)$.

The equality ker $\rho=\nu^{*} \oplus \mathbb{R}$ can be exploited to give another way to construct the homomorphism of equation (4.8) which underpins the cohomological criterion of Theorem 4.18. Denote the sheaf of basic smooth functions on $(P, \mathcal{F})$ by $\mathcal{C}_{\text {basic }}^{\infty}(P ; \mathcal{F})$, i.e., it consists of smooth functions which are locally constant on the leaves of $\mathcal{F}$. Equivalently, this can be defined as the sheaf of Casimirs of $(P, \Lambda)$, seeing as, in this case, a functions is basic for $\mathcal{F}$ if and only if it is a Casimir. There is a short exact sequence of sheaves

$$
1 \rightarrow \mathcal{C}_{\text {basic }}^{\infty}(P ; \mathcal{F}) \hookrightarrow \mathcal{C}^{\infty}(P) \stackrel{\mathrm{d}_{\mathcal{F}}}{\longrightarrow} \mathcal{Z}^{1}(\mathcal{F}) \rightarrow 1,
$$

where $\mathcal{C}^{\infty}(P)$ and $\mathcal{Z}^{1}(\mathcal{F})$ are the sheaves of smooth functions on $P$ and of closed foliated 1-forms respectively.

Lemma 5.9. The following is a commutative diagram of short exact sequences of sheaves

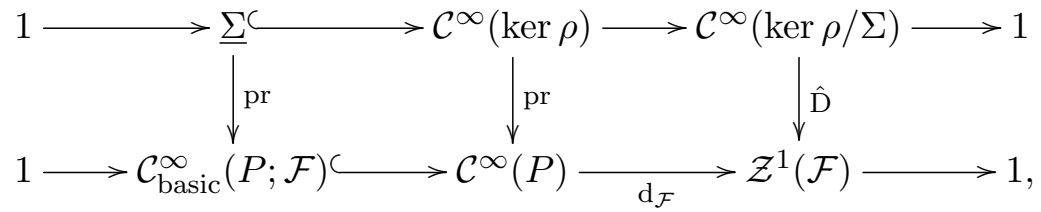

where pr denotes the homomorphism of sheaves induced by the projection pr: $\mathrm{T}^{*} P \oplus \mathbb{R} \rightarrow \mathbb{R}_{P}$, $\hat{\mathrm{D}}$ is the homomorphism of sheaves defined by equation (4.6), and $\underline{\Sigma}$ denotes the sheaf of smooth sections of $\Sigma \rightarrow P$.

Proof. First, observe that equation (5.5) implies that the image $\operatorname{pr}(\Sigma)$ of the sheaf homomorphism pr: $\mathcal{C}^{\infty}(\operatorname{ker} \rho) \rightarrow \mathcal{C}^{\infty}(P)$ lies in the sheaf $\mathcal{C}_{\text {basic }}^{\infty}(P ; \mathcal{F})$. The only non-trivial fact that needs checking is commutativity on the right hand side of the diagram (5.6). Let $[\alpha] \in \Gamma(\operatorname{ker} \rho / \Sigma)$ and set $\alpha=(\eta, f) \in \Gamma(\operatorname{ker} \rho)$ be a lift of $[\alpha]$. Then

$$
\hat{\mathrm{D}}([\alpha])=\left.\mathrm{D}(\eta, f)\right|_{\mathcal{F}}=\left.(\mathrm{d} f-\eta)\right|_{\mathcal{F}}=\mathrm{d}_{\mathcal{F}} f=\mathrm{d}_{\mathcal{F}} \circ \operatorname{pr}(\alpha),
$$

where the third equality follows from the fact that $\operatorname{ker} \rho=\nu^{*} \oplus \mathbb{R}$.

Since both $\mathcal{C}^{\infty}(P)$ and $\mathcal{C}^{\infty}(\operatorname{ker} \rho)$ are fine sheaves, there is a commutative diagram

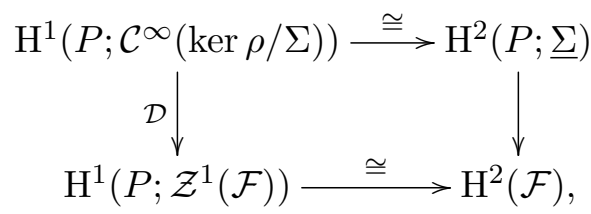


where the vertical maps are induced by the outer vertical maps of diagram (5.6) (cf. equation (4.8) also for the definition of $\mathcal{D}$ ), and the horizontal isomorphisms are the connecting morphisms induced by the short exact sequences in equation (5.6). Therefore, the following corollary holds.

Corollary 5.10. The map in cohomology

$$
\mathrm{H}^{2}(P ; \underline{\Sigma}) \rightarrow \mathrm{H}^{2}\left(P ; \mathcal{C}_{\text {basic }}^{\infty}(P ; \mathcal{F})\right) \cong \mathrm{H}^{2}(\mathcal{F})
$$

induced by the sheaf homomorphism $\operatorname{pr}: \underline{\Sigma} \rightarrow \mathcal{C}_{\text {basic }}^{\infty}(P ; \mathcal{F})$ equals $\mathcal{D}$ via the identifications of equation (5.7).

Corollary 5.10 indicates how to calculate the map $\mathcal{D}$, which is central to understanding whether a regular Poisson manifold $(P, \Lambda)$ with given transversal $\mathbb{Z}$-projective lattice $\Sigma \subset \nu^{*} \oplus \mathbb{R}$ admits a CIR (cf. Part (1) of Theorem 4.18).

Example 5.11. Suppose that $\Sigma=\mathbb{Z}\left\langle j^{1} f_{1}, \ldots, j^{1} f_{k}\right\rangle$ for some Casimirs $f_{i}$, so that it induces the trivial $\mathbb{Z}^{k}$-system of coefficients. Then (modulo torsion), an element in $\mathrm{H}^{2}(P ; \Sigma)$ can be written as

$$
\sum_{i=1}^{k}\left[\omega_{i}\right] \otimes j^{1} f_{i}
$$

where, for each $i, \omega_{i} \in \Omega^{2}(P)$ is a 2 -form with integral cohomology class. Since the map $\mathcal{D}: \mathrm{H}^{2}(P ; \underline{\Sigma}) \rightarrow \mathrm{H}^{2}(\mathcal{F})$ is induced by the projection pr: $\Sigma \rightarrow \mathcal{C}_{\text {basic }}^{\infty}(P ; \mathcal{F})$ and $\Sigma$ is a trivial bundle,

$$
\mathcal{D}\left(\sum_{i=1}^{k}\left[\omega_{i}\right] \otimes j^{1} f_{i}\right)=\sum_{i=1}^{k}\left[f_{i} \omega_{i}\right],
$$

where, for each $i, f_{i} \omega_{i}$ is a closed foliated 2 -form since $f_{i}$ is basic.

\subsection{Strong transversal $\mathbb{Z}$-affine structures are $\mathbb{Z}$-projective}

Before tackling the main problem of this section, it is necessary to show that, under some integrality condition (cf. Definition 5.13), transversal $\mathbb{Z}$-affine structures induce transversal $\mathbb{Z}$ projective structures. Let $(N, \mathcal{F})$ be a foliated manifold and recall the definitions of transversal affine and projective structures on $(N, \mathcal{F})$ (cf. Notes 4.13 and 5.4). The whole idea of this subsection hinges on the following well-known result, whose proof is included for completeness.

Lemma 5.12. Any transversal affine structure on $(N, \mathcal{F})$ induces a transversal projective structure on $(N, \mathcal{F})$.

Proof. Let $l \geq 0$ denote the codimension of $\mathcal{F}$. If $l=0$, there is nothing to prove, so suppose that $l \geq 1$. Consider the smooth map

$$
\begin{aligned}
\mathcal{I}: & \mathbb{R}^{l} \rightarrow \mathbb{R P}^{l}, \\
& \left(x_{1}, \ldots, x_{l}\right) \mapsto\left[x_{1}: \ldots: x_{l}: 1\right] ;
\end{aligned}
$$

this map is a diffeomorphism onto its image. Moreover, if

$$
\text { I: } \operatorname{Aff}\left(\mathbb{R}^{l}\right) \hookrightarrow \operatorname{GL}(l+1 ; \mathbb{R})
$$

$$
(A, \mathbf{b}) \mapsto\left(\begin{array}{cc}
A & \mathbf{b} \\
0 & 1
\end{array}\right)
$$


denotes the standard inclusion as groups, then for all $(A, \mathbf{b}) \in \operatorname{Aff}\left(\mathbb{R}^{l}\right)$ and for all $\mathbf{x} \in \mathbb{R}^{l}$,

$$
\mathcal{I}(A \mathbf{x}+\mathbf{b})=[\mathrm{I}(A, \mathbf{b})](\mathcal{I}(\mathbf{x})),
$$

where $[\cdot] \in \operatorname{PGL}(l+1 ; \mathbb{R})$ denotes the equivalence class of $\cdot \in \mathrm{GL}(l+1 ; \mathbb{R})$. Suppose that $\mathcal{A}=\left\{\left(U_{i}, \chi_{i}\right)\right\}$ is a transversal affine structure on $(N, \mathcal{F})$, and suppose that, for $i, j$ with $U_{i j} \neq \varnothing, h_{i j} \in \operatorname{Aff}\left(\mathbb{R}^{l}\right)$ are the transversal affine changes of coordinates as in Note 5.4. Then equation (5.9) implies that $\overline{\mathcal{A}}:=\left\{\left(U_{i}, \bar{\chi}_{i}\right)\right\}$, where $\bar{\chi}_{i}:=\mathcal{I} \circ \chi_{i}$ defines a transversal projective structure on $(N, \mathcal{F})$, the transversal projective changes of coordinates being $\bar{h}_{i j}=\left[\mathrm{I}\left(h_{i j}\right)\right]$.

Lemma 5.12 implies that any transversal $\mathbb{Z}$-affine structure induces a transversal projective structure; however, the latter need not be integral! Fundamentally, the reason is that, for any $l \geq 1, \mathrm{I}\left(\operatorname{Aff}_{\mathbb{Z}}\left(\mathbb{R}^{l}\right)\right) \not \subset \mathrm{GL}(l+1 ; \mathbb{Z})$, the issue being that the translational components of $\mathbb{Z}$-affine transformations are not necessarily integral. This leads to the following definition ${ }^{4}$, which can be thought of as an 'integrality condition' for a transversal $\mathbb{Z}$-affine structure.

Definition 5.13. A transversal $\mathbb{Z}$-affine structure $\mathcal{A}=\left\{\left(U_{i}, \chi_{i}\right)\right\}$ on $(N, \mathcal{F})$ is said to be strong if, for all $i, j$ with $U_{i j} \neq \varnothing, h_{i j}$ is (a restriction of) an element in $\operatorname{Aff}\left(\mathbb{Z}^{l}\right):=\operatorname{GL}(l ; \mathbb{Z}) \ltimes \mathbb{Z}^{l}$, where $l$ is the codimension of $\mathcal{F}$.

Corollary 5.14. Any strong transversal $\mathbb{Z}$-affine structure on $(N, \mathcal{F})$ induces a transversal $\mathbb{Z}$ projective structure on $(N, \mathcal{F})$.

Proof. Let $\mathcal{A}=\left\{\left(U_{i}, \chi_{i}\right)\right\}$ be a strong transversal $\mathbb{Z}$-structure on $(N, \mathcal{F})$ and, as in the proof of Lemma 5.12, let $h_{i j} \in \operatorname{Aff}\left(\mathbb{Z}^{l}\right)$ be the transversal $\mathbb{Z}$-affine changes of coordinates. Setting $A_{i j}:=\mathrm{I}\left(h_{i j}\right) \in \mathrm{GL}(l+1 ; \mathbb{Z})$, observe that $\left\{A_{i j}\right\}$ satisfies the cocycle condition, for $h_{i j}$ does. This fact can be used, together with Lemma 5.12, to prove that the atlas $\overline{\mathcal{A}}$ constructed in the proof of Lemma 5.12 defines a transversal $\mathbb{Z}$-projective structure (cf. Definition 4.12).

Henceforth, fix a strong transversal $\mathbb{Z}$-affine structure $\mathcal{A}=\left\{\left(U_{i}, \chi_{i}\right)\right\}$ on $(N, \mathcal{F})$ which, by Corollary 5.14 induces a transversal $\mathbb{Z}$-projective structure $\overline{\mathcal{A}}=\left\{\left(U_{i}, \bar{\chi}_{i}\right)\right\}$, which is also fixed. By Proposition 5.5, $\mathcal{A}$ corresponds to a full-rank lattice $\Xi \subset \nu^{*}$ whose sections are closed, where $\nu^{*}$ denotes the conormal bundle to $\mathcal{F}$. On the other hand, Proposition 4.14 ensures the existence of a line bundle $L \rightarrow N$ and a transversal $\mathbb{Z}$-projective lattice $\Sigma \subset J^{1} L$ satisfying conditions (T1)-(T3) in Definition 4.10 which corresponds to $\overline{\mathcal{A}}$. Seeing as $\overline{\mathcal{A}}$ is induced by $\mathcal{A}$, it is natural to ask what relation there is between $\Xi$ and $\Sigma$. To this end, it is useful to recall that, for each $i$,

- $\left.\Xi\right|_{U_{i}}=\chi_{i}^{*} \Xi^{l}$, where $\Xi^{l}:=\mathbb{Z}\left\langle\mathrm{d} x_{1}, \ldots, \mathrm{d} x_{l}\right\rangle \subset T^{*} \mathbb{R}^{l}$ is the standard $\mathbb{Z}$-affine structure on $\mathbb{R}^{l}$ (cf. Proposition 5.5);

- $\left.L\right|_{U_{i}}=\bar{\chi}_{i}^{*}(O(1))$ and $\left.\Sigma\right|_{U_{i}}=\bar{\chi}_{i}^{*} \Sigma^{l}$, where $O(1) \rightarrow \mathbb{R P}^{l}$ is the dual of the tautological line bundle and $\Sigma^{l} \subset J^{1}(O(1))$ is the $\mathbb{Z}$-projective lattice of Example 4.15 (cf. Proposition 4.14). In fact, since $\bar{\chi}_{i}=\mathcal{I} \circ \chi_{i}\left(\right.$ cf. the proof of Lemma 5.12), $\left.L\right|_{U_{i}}=\chi_{i}^{*}\left(\mathcal{I}^{*}(O(1))\right)$ and $\left.\Sigma\right|_{U_{i}}=$ $\chi_{i}^{*}\left(\mathcal{I}^{*}\left(\Sigma^{l}\right)\right)$.

Claim 5.15. The line bundle $L \rightarrow N$ is trivialisable.

Proof. First, observe that the line bundle $\mathcal{I}^{*}(O(1)) \rightarrow \mathbb{R}^{l}$ is trivialisable, for the restriction of $O(1)$ to $\mathcal{I}\left(\mathbb{R}^{l}\right)=\left\{\left[x_{1}, \ldots, x_{l}, x_{l+1}\right] \mid x_{l+1} \neq 0\right\}$ admits a nowhere vanishing section $\zeta$, namely the functional

$$
\begin{aligned}
& \mathbb{R}\left\langle x_{1}, \ldots, x_{l+1}\right\rangle \rightarrow \mathbb{R}, \\
& \mathbf{y}=\left(y_{1}, \ldots, y_{l+1}\right) \mapsto y_{l+1} .
\end{aligned}
$$

\footnotetext{
${ }^{4}$ The terminology in Definition 5.13 is not standard. For instance, [17] refers to the notion of strong (transversal) $\mathbb{Z}$-affine structure simply as (transversal) $\mathbb{Z}$-structure.
} 
The restriction of the natural action of $\operatorname{GL}(l+1 ; \mathbb{R})$ on $\Gamma(O(1))$ to $\mathrm{I}\left(\operatorname{Aff}\left(\mathbb{Z}^{l}\right)\right)$ fixes $\zeta$ : this is essentially because of the definition of the homomorphism I (cf. equation (5.8)). For each $i$, the section $\chi_{i}^{*} \circ \mathcal{I}^{*} \zeta$ of $\left.L\right|_{U_{i}}$ is nowhere vanishing. If $U_{i j} \neq \varnothing$, then

$$
\left.\left(\chi_{j}^{*} \circ \mathcal{I}^{*} \zeta\right)\right|_{U_{i j}}=\left.\left(\chi_{i}^{*} \circ h_{i j}^{*} \circ \mathcal{I}^{*} \zeta\right)\right|_{U_{i j}}=\left.\left(\chi_{i}^{*} \circ \mathcal{I}^{*} \circ \mathrm{I}\left(h_{i j}\right)^{*} \circ \zeta\right)\right|_{U_{i j}}=\left.\left(\chi_{i}^{*} \circ \mathcal{I}^{*} \zeta\right)\right|_{U_{i j}},
$$

where the second equality follows from equation (5.9) and the last one by the fact that $\zeta$ is fixed by $\mathrm{I}\left(\operatorname{Aff}\left(\mathbb{Z}^{l}\right)\right)$. Therefore, $L \rightarrow N$ admits a globally defined nowhere vanishing section, thus proving that it is trivialisable.

Henceforth, fix the trivialisations $\mathcal{I}^{*}(O(1)) \cong \mathbb{R}_{\mathbb{R}^{l}}$ and $L \cong \mathbb{R}_{N}$ induced as in the proof of Claim 5.15 unless otherwise stated. Since $L \rightarrow N$ is trivial, $\Sigma \subset J^{1} \mathbb{R}_{N}$ is a full-rank lattice of $\nu^{*} \oplus \mathbb{R}$ (cf. Example 4.11) all of whose sections are holonomic (cf. condition (T3)).

Proposition 5.16. If $\mathrm{P}: \nu^{*} \oplus \mathbb{R} \rightarrow \nu^{*}$ denotes projection onto the first factor, then $\mathrm{P}(\Sigma)=\Xi$.

Proof. First, it is shown that if $\mathrm{P}_{\mathbb{R}^{l}}: T^{*} \mathbb{R}^{l} \oplus \mathbb{R}=J^{1} \mathbb{R}_{\mathbb{R}^{l}} \rightarrow T^{*} \mathbb{R}^{l}$ is the projection onto the first factor, then $\mathrm{P}_{\mathbb{R}^{l}}\left(\mathcal{I}^{*}\left(\Sigma^{l}\right)\right)=\Xi^{l}$. This follows basically by unravelling the definitions of $\Sigma^{l}$ and $\Xi^{l}$. By definition of $\Sigma^{l}$ and of $\mathcal{I}$ (cf. Example 4.15 and the proof of Lemma 5.12),

$$
\mathcal{I}^{*} \Sigma^{l}=\mathbb{Z}\left\langle j^{1} x_{1}, \ldots, j^{1} x_{l}, j^{1} 1\right\rangle,
$$

where, for each $j, x_{j}$ denotes the functional which assigns to a point in $\mathbb{R}^{l}$ its $j$-th coordinate. Writing, for each $j, j^{1} x_{l}=\left(\mathrm{d} x_{l}, x_{l}\right) \in \Gamma\left(T^{*} \mathbb{R}^{l} \oplus \mathbb{R}\right)$, and $j^{1} 1=(0,1)$, it is clear that $\mathrm{P}_{\mathbb{R}^{l}}\left(\mathcal{I}^{*}\left(\Sigma^{l}\right)\right)=\Xi^{l}$. The general case follows immediately by observing that, for all $i$, the restrictions $\left.\mathrm{P}\right|_{U_{i}}:\left.\left.\left(\nu^{*} \oplus \mathbb{R}\right)\right|_{U_{i}} \rightarrow \nu^{*}\right|_{U_{i}},\left.\Sigma\right|_{U_{I}}$ and $\left.\Xi\right|_{U_{i}}$ are simply the pull-backs along $\chi_{i}$ of $\mathrm{P}_{\mathbb{R}^{l}}, \mathcal{I}^{*} \Sigma^{l}$ and $\Xi^{l}$ respectively, where, as above, $\mathcal{A}=\left\{\left(U_{i}, \chi_{i}\right)\right\}$ is the fixed transversal $\mathbb{Z}$-affine structure on $(N, \mathcal{F})$.

Note 5.17. A closer look at the proof of Proposition 5.16 yields a slightly stronger result. Observe that $\mathcal{I}^{*} \Sigma^{l}$ contains a one-dimensional sub-lattice $\mathbb{Z}\left\langle j^{1} 1\right\rangle$ which is precisely the kernel of $\mathrm{P}_{\mathbb{R}^{l}}: \mathcal{I}^{*} \Sigma^{l} \rightarrow \Xi^{l}$. The locally defined one-dimensional sub-lattices $\left.\chi_{i}^{*}\left(\mathbb{Z}\left\langle j^{1} 1\right\rangle\right) \subset \Sigma\right|_{U_{i}}$ patch together to yield a globally defined, trivial one-dimensional sub-lattice of $\Sigma$ which is precisely the kernel of $\mathrm{P}: \Sigma \rightarrow \Xi$ (where, by abuse of notation, the restrictions of $\mathrm{P}_{\mathbb{R}^{l}}$ and of $\mathrm{P}$ to their respective lattices are also denoted by $\mathrm{P}_{\mathbb{R}^{l}}$ and $\mathrm{P}$ respectively). One invariant way to see this is to observe that one globally defined section of $\Sigma$ is the first jet of the nowhere vanishing section of $L \rightarrow N$. Under the trivialisation $L \cong \mathbb{R}_{N}$ induced by Claim 5.15, this section corresponds to the function 1 ; in other words, ker $\mathrm{P}=\mathbb{Z}\left\langle j^{1} 1\right\rangle$. The projection $\mathrm{P}$ and its restriction to $\Sigma$ induces the following commutative diagram of short exact sequences of bundle maps

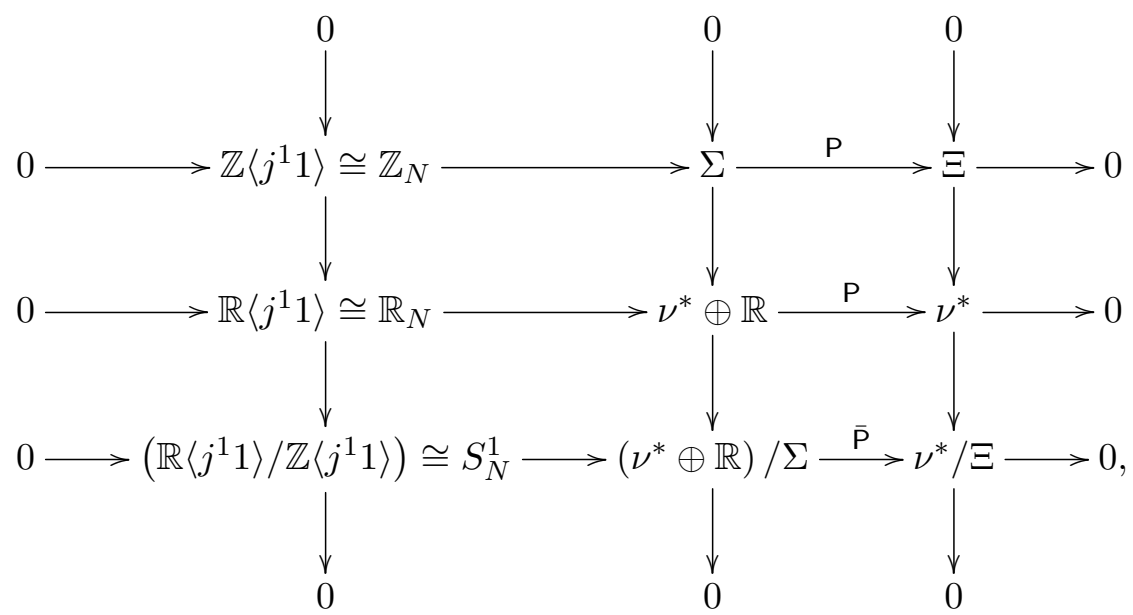


where $S_{N}^{1} \rightarrow N$ is the trivial $S^{1}$-bundle over $N$ and $\overline{\mathrm{P}}:\left(\nu^{*} \oplus \mathbb{R}\right) / \Sigma \rightarrow \nu^{*} / \Xi$ is the projection induced by $\mathrm{P}$.

By Proposition 5.16 and its proof, there is a homomorphism of sheaves

$$
\begin{aligned}
\underline{\mathrm{P}}: & \underline{\Sigma} \rightarrow \Xi, \\
& j^{1} f \mapsto \mathrm{d} f,
\end{aligned}
$$

where $\underline{\Sigma}$ and $\Xi$ denote the sheaves of sections of $\Sigma \rightarrow N$ and $\Xi \rightarrow N$ respectively.

Lemma 5.18. There is a commutative diagram of short exact sequences of sheaves

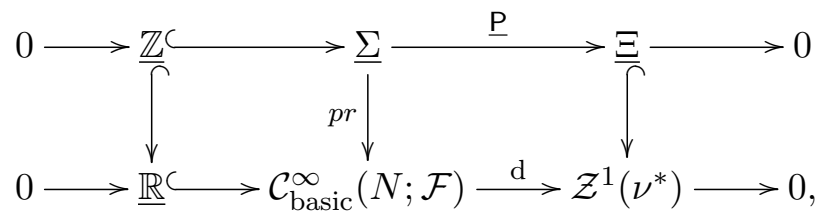

where $\underline{\mathbb{Z}}, \mathbb{R}$ are the sheaf of sections of $\mathbb{Z}_{N}$ and $\mathbb{R}_{N}$ respectively, $\mathcal{C}_{\text {basic }}^{\infty}(N ; \mathcal{F})$ is the sheaf of basic functions, $\mathcal{Z}^{1}\left(\nu^{*}\right)$ is the sheaf of closed 1 -forms which vanish along $\mathcal{F}, \operatorname{pr}: \underline{\Sigma} \rightarrow \mathcal{C}_{\text {basic }}^{\infty}(N ; \mathcal{F})$ is the homomorphism induced by the projection $\nu^{*} \oplus \mathbb{R} \rightarrow \mathbb{R}_{N}$, and $\mathrm{d}: \mathcal{C}_{\text {basic }}^{\infty}(N ; \mathcal{F}) \rightarrow \mathcal{Z}^{1}\left(\nu^{*}\right)$ is induced by taking derivatives.

Proof. The top row is a short exact sequence as it is induced by the top short exact sequence of bundles of equation (5.10), while it is well-known that the bottom row is a short exact sequence. The projection pr: $\nu^{*} \oplus \mathbb{R} \rightarrow \mathbb{R}_{N}$ induces a homomorphism of sheaves of sections pr: $\mathcal{C}^{\infty}\left(\nu^{*} \oplus \mathbb{R}\right) \rightarrow \mathcal{C}^{\infty}(N)$; therefore, a priori, the codomain of its restriction to $\Sigma$ is $\mathcal{C}^{\infty}(N)$. However, $\Sigma$ is a transversal $\mathbb{Z}$-projective structure on $(N, \mathcal{F})$ and, therefore, all its locally defined sections are holonomic. Since $\Sigma \subset \nu^{*} \oplus \mathbb{R}$, it follows that if $j^{1} f \in \Gamma_{\text {loc }}(\Sigma)$, then $\operatorname{pr}\left(j^{1} f\right)=f$ is basic (cf. the implications in equation (5.5)). Therefore the middle vertical homomorphism of equation (5.11) is well-defined. Commutativity of the diagram of equation (5.11) follows by definition of $\underline{P}$.

Consider the long exact sequences in cohomology associated to the top and bottom rows of equation (5.11). The one induced by the bottom short exact sequence is well-known to be

$$
\cdots \longrightarrow \mathrm{H}^{2}(N ; \mathbb{R}) \longrightarrow \mathrm{H}^{2}(\mathcal{F}) \longrightarrow \mathrm{H}_{\mathrm{rel}}^{3}(N ; \mathcal{F}) \longrightarrow \mathrm{H}^{3}(N ; \mathbb{R}) \longrightarrow \cdots,
$$

where the map $\mathrm{H}^{2}(\mathcal{F}) \rightarrow \mathrm{H}_{\text {rel }}^{3}(N ; \mathcal{F})$ sends the cohomology class of a foliated 2 -form to the cohomology class of the exterior differential of any of its extensions as in Note 5.2 (cf. [4, 15] for details of the above short exact sequence). Lemma 5.18 implies that there is the following commutative diagram

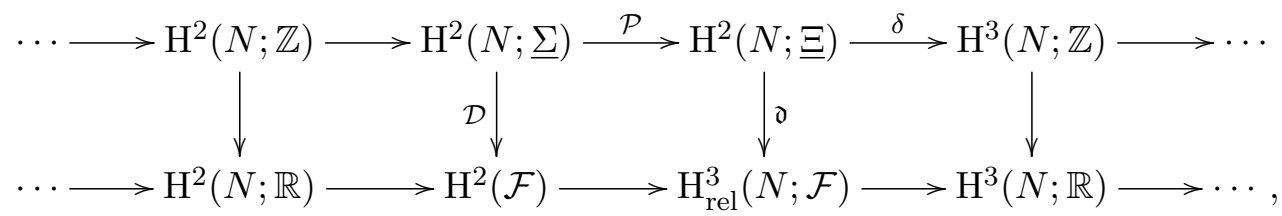

where

- $\mathrm{H}^{*}(N ; \underline{\mathbb{Z}}), \mathrm{H}^{*}(N ; \underline{\mathbb{R}})$ are identified with the singular cohomology groups $\mathrm{H}^{*}(N ; \mathbb{Z})$ and $\mathrm{H}^{*}(N ; \mathbb{R})$ respectively, and the maps $\mathrm{H}^{*}(N ; \mathbb{Z}) \rightarrow \mathrm{H}^{*}(N ; \mathbb{R}) \cong \mathrm{H}^{*}(N ; \mathbb{Z}) \otimes_{\mathbb{Z}} \mathbb{R}$ send $\mathrm{H}^{*}(N ; \mathbb{Z})$ to $\mathrm{H}^{*}(N ; \mathbb{Z}) \otimes_{\mathbb{Z}} 1 ;$ 
- $\mathcal{P}: \mathrm{H}^{2}(N ; \underline{\Sigma}) \rightarrow \mathrm{H}^{2}(N ; \Xi)$ is induced by $\underline{\mathrm{P}}: \underline{\Sigma} \rightarrow \underline{\Xi} ;$

- the maps $\mathcal{D}: \mathrm{H}^{2}(N ; \underline{\Sigma}) \rightarrow \mathrm{H}^{2}(\mathcal{F})$ and $\mathfrak{d}: \mathrm{H}^{2}(N ; \Xi) \rightarrow \mathrm{H}_{\text {rel }}^{3}(N ; \mathcal{F})$ are the homomorphisms constructed in Sections 4.3 and 5.1 respectively (cf. Corollary 5.10 and Note 5.7).

Note 5.19. The homomorphism $\mathcal{P}: \mathrm{H}^{2}(N ; \underline{\Sigma}) \rightarrow \mathrm{H}^{2}(N ; \Xi)$ has a clear geometric interpretation. Suppose that $\phi: M \rightarrow N$ is a principal $\left(\nu^{*} \oplus \mathbb{R}\right) / \Sigma$-bundle with Chern class $c \in \mathrm{H}^{2}(N ; \underline{\Sigma})$; then there is a free and proper action $\left(\nu^{*} \oplus \mathbb{R}\right) / \Sigma \curvearrowright M$ along $\phi$. Restricting this action to the subbundle $\left(\mathbb{R}\left\langle j^{1} 1\right\rangle / \mathbb{Z}\left\langle j^{1} 1\right\rangle\right) \cong S_{N}^{1} \subset\left(\nu^{*} \oplus \mathbb{R}\right) / \Sigma$ induces a free and proper $S^{1}$-action on $M$. Set $S:=M / S^{1}$. Since the $S^{1}$-action is tangent to the fibres of $\phi$, there is a uniquely defined smooth map $\Phi: S \rightarrow N$ along which the quotient $\left(\left(\nu^{*} \oplus \mathbb{R}\right) / \Sigma\right) /\left(\mathbb{R}\left\langle j^{1} 1\right\rangle / \mathbb{Z}\left\langle j^{1} 1\right\rangle\right)=\nu^{*} / \Xi$ acts freely and properly. Thus $\Phi: S \rightarrow N$ is a principal $\nu^{*} / \Xi$-bundle whose Chern class is precisely $\mathcal{P}(c)$.

\subsection{Symplectic vs contact isotropic realisations of Poisson manifolds}

Suppose that $(P, \Lambda)$ is a regular Poisson manifold and let $\Xi \subset \nu^{*}$ be a strong transversal $\mathbb{Z}$-affine lattice on its symplectic foliation $\mathcal{F}$. Using the notation of Section 5.3 , let $\Sigma \subset \nu^{*} \oplus \mathbb{R}$ denote the transversal $\mathbb{Z}$-projective lattice induced by Corollary 5.14. Then the following realisability criterion holds.

Theorem 5.20. The regular Poisson manifold $(P, \Lambda)$ admits a CIR with period lattice $\Sigma$ if and only if it admits a SIR $\Phi:(S, \omega) \rightarrow(P, \Lambda)$ with period lattice $\Xi$ with the property that $\omega$ is integral.

Proof. $(\Rightarrow)$ : Suppose that $(P, \Lambda)$ admits a CIR $\phi:(M, H) \rightarrow(P, \Lambda)$ with period lattice $\Sigma$. As in Note 5.19, consider the free and proper $S^{1}$-action on $(M, H)$ arising from restricting the $\left(\nu^{*} \oplus \mathbb{R}\right) / \Sigma$-action on $(M, H)$ to $\mathbb{R}\left\langle j^{1} 1\right\rangle / \mathbb{Z}\left\langle j^{1} 1\right\rangle \cong S_{P}^{1}$, and denote the resulting quotient map by $\Pi: M \rightarrow S:=M / S^{1}$. There is an induced surjective submersion $\Phi: S \rightarrow P$ (which is a principal $\nu^{*} / \Xi$-bundle by Note 5.19 ). The aim is to show that $S$ inherits an integral symplectic form from $(M, H)$. Since $(P, \Lambda)$ defines a Jacobi structure on the trivial line bundle $\mathbb{R}_{P}$, it follows that there is a contact form $\theta \in \Omega^{1}(M)$ with $H=\operatorname{ker} \theta$. Moreover, $\theta$ can be chosen so that the associated Reeb vector field $R_{1}$ is precisely the generator of the above principal $S^{1}$-action; this is because of the definition of the action $\left(\nu^{*} \oplus \mathbb{R}\right) / \Sigma \curvearrowright(M, H)$ and since the $S^{1}$-action arises by restricting to $\mathbb{R}\left\langle j^{1} 1\right\rangle / \mathbb{Z}\left\langle j^{1} 1\right\rangle$. Thus $\theta$ is a connection 1 -form for the principal bundle $\Pi: M \rightarrow S$ and, since it is a contact form, its curvature $\omega \in \Omega^{2}(S)$ is, in fact, an integral symplectic form. To summarise the above discussion, the following diagram commutes

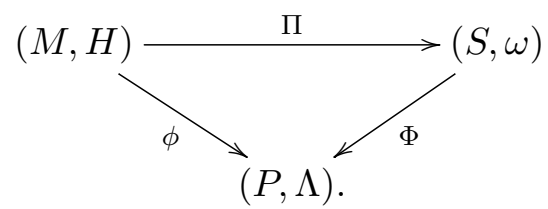

It remains to show that $\Phi:(S, \omega) \rightarrow(P, \Lambda)$ is a SIR with period lattice $\Xi$. First, observe that $\phi$ and $\Pi$ being Jacobi maps, and $\Pi$ being a submersion, imply that $\Phi$ is Poisson map. To see that the fibres of $\Phi$ are isotropic, observe that $D \Pi(\operatorname{ker} D \phi \cap H)=\operatorname{ker} D \Phi$. The condition of $\phi:(M, H) \rightarrow(P, \Lambda)$ being a contact isotropic realisation translates into ker $D \phi \cap H \subset H$ being isotropic for $\mathrm{d} \theta$ (cf. Note 3.3). The fact that $\mathrm{d} \theta=\Pi^{*} \omega$ thus implies that the fibres of $\Phi$ are isotropic. If $\rho_{M}: J^{1} M \rightarrow T M$ and $\rho_{S}: T^{*} S \rightarrow T S$ denote the anchor maps associated to the contact and symplectic structures on $M$ and $S$ respectively, then the fact that $\Pi$ is a Jacobi map and commutativity of the diagram in equation (5.13) imply that, for all $(\alpha, f) \in \Gamma\left(\nu^{*} \oplus \mathbb{R}\right)$,

$$
D \Pi\left(\rho_{M}\left(\phi^{*}(\alpha, f)\right)\right)=\rho_{S}\left(\Phi^{*} \alpha\right) \circ \Pi .
$$


In other words, the vector fields $\rho_{M}\left(\phi^{*}(\alpha, f)\right)$ and $\rho_{S}\left(\Phi^{*} \alpha\right)$ are $\Pi$-related; therefore, for all $t \in \mathbb{R}, \Pi \circ \varphi_{(\alpha, f)}^{t}=\varphi_{\alpha}^{t} \circ \Pi$, where $\varphi_{(\alpha, f)}^{t}$ and $\varphi_{\alpha}^{t}$ denote the flows at time $t$ of $\rho_{M}\left(\phi^{*}(\alpha, f)\right)$ and $\rho_{S}\left(\Phi^{*} \alpha\right)$ respectively. Thus if $(\alpha, f) \in \Gamma(\Sigma)$, then $\alpha$ is a section of the isotropy bundle of the $\nu^{*}$-action on $\Phi:(S, \omega) \rightarrow(P, \Lambda)$; however, $\alpha=\mathrm{P}(\alpha, f)$, where $\mathrm{P}: \nu^{*} \oplus \mathbb{R} \rightarrow \nu^{*}$ is the projection of equation (5.11). Therefore, $\Xi=\mathrm{P}(\Sigma)$ (cf. Proposition 5.16) is contained in the isotropy $\hat{\Xi}$ of the action $\nu^{*} \hookrightarrow(S, \omega)$ along $\Phi$. To show that $\Xi=\hat{\Xi}$, observe that if $\alpha$ is a local section of $\hat{\Xi}$ defined on a sufficiently small domain $U \subset P$, then $\alpha=\mathrm{d} f$ for some locally defined smooth function $f$ on $P$. Then have that $\Pi \circ \varphi_{(\mathrm{d} f, f)}^{1}=\varphi_{\mathrm{d} f}^{1} \circ \Pi=\Pi$, so that $\varphi_{(\mathrm{d} f, f)}^{1}(m) \in \Pi^{-1}(\Pi(m))$ for all $m \in \phi^{-1}(U)$. Since ker $D \Pi=\mathbb{R}\left\langle R_{1}\right\rangle$, there exists a smooth function $\tau: \phi^{-1}(U) \rightarrow \mathbb{R} / \mathbb{Z}$ such that, for all $m \in \phi^{-1}(U), \varphi_{(\mathrm{d} f, f)}^{1}(m)=\varphi_{(0,1)}^{\tau(m)}(m)$.

Claim 5.21. The function $\tau$ is $\phi$-basic.

Proof of Claim 5.21. The above statement is proved below by showing that $\tau$ is constant along the fibres of $\phi$. Fix $m \in \phi^{-1}(U)$ and let $m^{\prime} \in \phi^{-1}(\phi(m))$. Since the action of $\nu^{*} \oplus \mathbb{R}$ on $\phi:(M, H) \rightarrow(P, \Lambda)$ is transitive along the fibres (cf. Note 4.5), there exists an element $\xi \in\left(\nu^{*} \oplus \mathbb{R}\right)_{\phi(m)}$ such that $m^{\prime}=\varphi_{\xi}^{1}(m)$. Then

$$
\begin{aligned}
\varphi_{(0,1)}^{\tau\left(\varphi_{\xi}^{1}(m)\right)}\left(\varphi_{\xi}^{1}(m)\right) & =\varphi_{(\mathrm{d} f, f)}^{1} \circ \varphi_{\xi}^{1}(m)=\varphi_{\xi}^{1} \circ \varphi_{(\mathrm{d} f, f)}^{1}(m)=\varphi_{\xi}^{1} \circ \varphi_{(0,1)}^{\tau(m)}(m) \\
& =\varphi_{\xi}^{1} \circ \varphi_{(0, \tau(m))}^{1}(m)=\varphi_{(0, \tau(m))}^{1}\left(\varphi_{\xi}^{1}(m)\right) \\
& =\varphi_{(0,1)}^{\tau(m)}\left(\varphi_{\xi}^{1}(m)\right),
\end{aligned}
$$

where the second and fourth equalities follow from the fact that the vector fields $\rho_{M}\left(\phi^{*} \xi\right)$, $\rho_{M}\left(\phi^{*}(\mathrm{~d} f, f)\right)$ and $\rho_{M}\left(\phi^{*}(0,1)\right)$ commute, as $\xi,(\mathrm{d} f, f)_{\phi(m)},(0,1)_{\phi(m)}$ are elements of $\left(\nu^{*} \oplus\right.$ $\mathbb{R})_{\phi(m)}$, which is an abelian Lie algebra.

Let $\bar{\tau}$ be the unique smooth function such that $\tau=\phi^{*} \bar{\tau}$. Since $R_{1}$ is tangent to the fibres of $\phi$, it follows that $\varphi_{(0,1)}^{\tau}=\varphi_{(0, \bar{\tau})}^{1}$. Thus, on $\phi^{-1}(U), \varphi_{(\mathrm{d} f, f)}^{1} \circ \varphi_{(0,-\bar{\tau})}^{1}=i d$., thus showing that $(\mathrm{d} f, f-\bar{\tau})$ is a local section of $\Sigma$; this immediately implies that $\mathrm{d} f$ is a local section of $\mathrm{P}(\Sigma)=\Xi$, thus proving that $\Xi=\bar{\Xi}$.

$(\Leftarrow)$ : Conversely, suppose that $\Phi:(S, \omega) \rightarrow(M, H)$ is a SIR with period lattice $\Xi$, where $\omega$ is an integral symplectic form. Let $\Pi: M \rightarrow S$ be a principal $S^{1}$-bundle with Chern class equal to $[\omega] \in \mathrm{H}^{2}(S ; \mathbb{Z})$; a connection 1-form $\theta \in \Omega^{1}(M)$ with curvature $\omega$ defines a contact structure $H=\operatorname{ker} \theta$ on $M$ with respect to which $\Pi:(M, H) \rightarrow(S, \omega)$ is a CIR (cf. Example 3.4). The aim is to show that the composite $\phi:=\Phi \circ \Pi:(M, H) \rightarrow(P, \Lambda)$ is a CIR with period lattice equal to $\Sigma$. Since $\phi=\Phi \circ \Pi$ and $\Pi$ and $\Phi$ are Jacobi maps, $\phi$ is a Jacobi map. Given that $\operatorname{ker} D \Pi=\mathbb{R}\left\langle R_{1}\right\rangle$ and that $\operatorname{ker} D \Pi \subset \operatorname{ker} D \phi$, it follows that ker $D \phi$ is transversal to $H$. Observe that, by definition of $\theta$, for any $m \in M,\left.D_{m} \Pi\right|_{H_{m}}: H_{m} \rightarrow T_{\Pi(m)} S$ is an isomorphism of symplectic vector spaces which identifies $\operatorname{ker} D \phi \cap H$ with $\operatorname{ker} D \Phi$. Since the latter is isotropic, so is the former; by Note 3.3, it follows that $\phi:(M, H) \rightarrow(P, \Lambda)$ is a CIR. It remains to show that the period lattice of $\phi$ is precisely $\Sigma$. Denote the isotropy of the $\nu^{*} \oplus \mathbb{R}$-action on $\phi:(M, H) \rightarrow(P, \Lambda)$ by $\hat{\Sigma}$; observe that, by construction, $\mathbb{Z}\left\langle j^{1} 1\right\rangle \subset \hat{\Sigma}$, as the Reeb vector field $R_{1}$ is the infinitesimal generator of the free and proper $S^{1}$-action on $M$. Using notation from $(\Rightarrow)$-part of the proof, have that, for all $(\alpha, f) \in \Gamma\left(\nu^{*} \oplus \mathbb{R}\right)$, and for all $t \in \mathbb{R}$,

$$
\Pi \circ \varphi_{(\alpha, f)}^{t}=\varphi_{\alpha}^{t} \circ \Pi .
$$

Thus, if $j^{1} f \in \Gamma_{\text {loc }}(\hat{\Sigma})$, then $\mathrm{P}\left(j^{1} f\right)=\mathrm{d} f \in \Gamma(\Xi)$. Hence $\hat{\Sigma} \subset \Sigma$. To show that, in fact $\hat{\Sigma}=\Sigma$, the idea is to argue as above. Consider a local section $\xi \in \Sigma$; since $\mathrm{P}(\Sigma)=\Xi$ and by equation (5.14), there is a locally defined smooth function $\tau$ such that $\varphi_{\xi}^{1}=\varphi_{(0,1)}^{\tau}$. Arguing 
as in Claim 5.21, there exists a locally defined smooth function $\bar{\tau}$ on $P$ with $\tau=\phi^{*} \bar{\tau}$. Thus, $(0, \bar{\tau}) \in \Gamma\left(\nu^{*} \oplus \mathbb{R}\right)$ and $\xi-(0, \bar{\tau}) \in \Gamma(\hat{\Sigma})$. Using the fact $\hat{\Sigma} \subset \Sigma$, obtain that $\bar{\tau} \in \mathbb{Z}$, which therefore implies that $\xi \in \Gamma(\hat{\Sigma})$, since $\mathbb{Z}\left\langle j^{1} 1\right\rangle \subset \hat{\Sigma}$. This concludes the proof.

Note 5.22. Suppose that $(P, \Lambda)$ admits a CIR with period lattice $\Sigma$, say with Chern class $c$; commutativity of the diagram of equation (5.12) implies that $\mathcal{P}(c)$ is the Chern class of a SIR of $(P, \Lambda)$ with period lattice $\Xi$. However, Theorem 5.20 shows that the symplectic form on the total space of this SIR can be chosen to be integral, something that, to the best of our knowledge, cannot be inferred simply from the commutativity of the diagram (5.12). Conversely, suppose that $c^{\prime} \in \mathrm{H}^{2}(P ; \Xi)$ is the Chern class of a SIR of $(P, \Lambda)$ with period lattice $\Xi$. Commutativity of the diagram of equation (5.12) implies that a necessary condition for the existence of a CIR of $(P, \Lambda)$ with period lattice $\Sigma$ is that $\delta\left(c^{\prime}\right)=0$, as the characteristic class $v=\mathfrak{d}\left(c^{\prime}\right)$ is defined by an exact 3 -form, thus being mapped to zero under $\mathrm{H}_{\text {rel }}^{3}(P ; \mathcal{F}) \rightarrow \mathrm{H}^{3}(P ; \mathbb{R})$. This is, however, a very mild restriction: if $\mathrm{H}^{3}(P ; \mathbb{Z})$ is torsion-free, $\delta\left(c^{\prime}\right)=0$ is automatic, as the vertical map $\mathrm{H}^{3}(P ; \mathbb{Z}) \rightarrow \mathrm{H}^{3}(P ; \mathbb{R})$ in equation (5.12) is the natural inclusion. If, on the one hand, $\delta\left(c^{\prime}\right)=0$ implies that there exists $c \in \mathrm{H}^{2}(P ; \underline{\Sigma})$ with $\mathcal{P}(c)=c^{\prime}$ by exactness of the top row of $(5.12)$, on the other, this is not sufficient to conclude that $c$ is the Chern class of a CIR of $(P, \Lambda)$ with period lattice $\Sigma$. Intuitively, what is needed is that the foliated 2 -form $\omega_{\mathcal{F}}$ be, in some sense, integral. For instance, if $v=0$, i.e., there exists a globally defined closed 2-form $\Omega \in \Omega^{2}(P)$ extending $\omega_{\mathcal{F}}$, then integrality of $\Omega$ suffices to guarantee, together with $\delta\left(c^{\prime}\right)=0$, that there exists a CIR of $(P, \Lambda)$ with period lattice $\Sigma$.

Example 5.23. (For details about this example, see [6, Section 4.5.1].) Let $G$ be a compact, simply connected Lie group and set $\mathfrak{g}=\operatorname{Lie}(G)$. The subset $\mathfrak{g}_{\text {reg }}^{*} \subset \mathfrak{g}^{*}$ consisting of coadjoint orbits whose stabiliser is a maximal torus is a regular Poisson manifold, whose bracket is the restriction of the linear bracket on $\mathfrak{g}^{*}$. In fact, if $\mathbb{T} \subset G$ is a maximal torus with $\mathfrak{t}=\operatorname{Lie}(\mathbb{T})$, and $\mathfrak{c} \subset \mathfrak{t}^{*}$ is the interior of a Weyl chamber, then there is a diffeomorphism $\mathfrak{g}_{\text {reg }}^{*} \cong G / \mathbb{T} \times \mathfrak{c}$, which identifies $\mathfrak{c}$ as the leaf space of the symplectic foliation induced by the above Poisson structure. The kernel of exp: $\mathfrak{t} \rightarrow \mathbb{T}$ induces a $\mathbb{Z}$-affine structure on $\mathfrak{t}^{*}$, which, upon suitable identifications, corresponds to the standard $\mathbb{Z}$-affine structure on $\mathbb{R}^{l} \cong \mathfrak{t}^{*}$, where $l=\operatorname{rk} G$. This $\mathbb{Z}$-affine structure is intimately connected to the Poisson geometry of $\mathfrak{g}_{\text {reg }}^{*}$ (cf. [6, Section 4.5.1]). Fix $\xi_{0} \in \mathfrak{c}$ and denote by $S_{0} \subset \mathfrak{g}_{\text {reg }}^{*}$ the coadjoint orbit through $\xi_{0}$. Viewing $\mathfrak{c}$ as an open subset of $\mathfrak{t}^{*}$, obtain a strong transversal $\mathbb{Z}$-affine lattice $\Xi$ on $\mathfrak{g}_{\text {reg }}^{*}$ and denote by $\Sigma$ the transversal $\mathbb{Z}$-projective lattice on $\mathfrak{g}_{\mathrm{reg}}^{*}$ induced as in Corollary 5.14. If $\xi^{1}, \ldots, \xi^{l}: \mathfrak{c} \rightarrow \mathbb{R}$ denote $\mathbb{Z}$-affine coordinates on $\mathfrak{c}$, then $\Xi=\mathbb{Z}\left\langle\mathrm{d} \xi^{1}, \ldots \mathrm{d} \xi^{l}\right\rangle$ and $\Sigma=\mathbb{Z}\left\langle j^{1} \xi^{1}, \ldots, j^{1} \xi^{l}, j^{1} 1\right\rangle$, where, by abuse of notation, $\xi^{1}, \ldots \xi^{l}$ are seen as functions on $\mathfrak{g}_{\mathrm{reg}}^{*}$. On the other hand, since $G$ is compact, if $\mathcal{F}$ denotes the symplectic foliation on $\mathfrak{g}_{\text {reg }}^{*}$, then $\mathrm{H}^{2}(\mathcal{F}) \cong \mathrm{H}^{2}\left(S_{0} ; \mathbb{R}\right) \otimes C^{\infty}(\mathfrak{c})$ (cf. [15]). If $c_{1}, \ldots, c_{l} \in \mathrm{H}^{2}\left(S_{0} ; \mathbb{Z}\right)$ are torsion-free generators, then [6, Remark 4.3.5] implies that the foliated class of the foliated symplectic form $\omega_{\mathcal{F}}$ is given by

$$
\left[\omega_{\mathcal{F}}\right]=\left[\omega_{0}\right]+\sum_{i=1}^{l} \xi^{i} \otimes c_{i},
$$

where $\left[\omega_{0}\right] \in \mathrm{H}^{2}\left(S_{0} ; \mathbb{R}\right)$ is the cohomology class of the symplectic form on $S_{0}$ (cf. [6, Section 4.5.1] for a reason why $\left.\operatorname{rk~H}^{2}\left(S_{0} ; \mathbb{Z}\right)=\operatorname{rk} G\right)$. Seeing as $S_{0}$ can be chosen so that [ $\left.\omega_{0}\right]$ is integral, Example 5.11 implies that $\mathfrak{g}_{\text {reg }}^{*}$ admits a CIR with period lattice $\Sigma$ and, equivalently by Theorem 5.20 , a SIR with period lattice $\Xi$ whose total space has an integral symplectic form.

Observe that choosing $S_{0}$ so that it has an integral symplectic form is equivalent to having $\left(\xi^{1}\left(\xi_{0}\right), \ldots, \xi^{l}\left(\xi_{0}\right)\right) \in \mathbb{Z}^{l}$. Let $U \subset \mathfrak{c}$ be an open, connected subset not containing any integral point (with respect to the above strong $\mathbb{Z}$-affine structure!) and consider the open Poisson submanifold $\left(P_{U}, \Lambda_{U}\right) \subset \mathfrak{g}_{\text {reg }}^{*}$ obtained by considering the union of coadjoint orbits corresponding 
to points in $U$. Denote the induced transversal strong $\mathbb{Z}$-affine and $\mathbb{Z}$-projective lattices by $\Xi_{U}$ and $\Sigma_{U}$ respectively. Observe that $\Sigma_{U}$ equals the transversal $\mathbb{Z}$-projective lattice obtained from $\Xi_{U}$ as in Corollary 5.14. Example 5.11 implies that $\left(P_{U}, \Lambda_{U}\right)$ admits no CIR with period lattice $\Sigma_{U}$, but it admits a SIR with period lattice $\Xi_{U}$ as it can be readily verified using [11, Section 4].

\section{A Properties of regular Jacobi manifolds all of whose leaves are even dimensional}

Lemma A.1. Let $(P, L,\{\cdot, \cdot\})$ be a regular Jacobi manifold with even dimensional leaves. The connection $\bar{\nabla}$ of equation (3.4) is a well-defined flat $T \mathcal{F}$-connection.

Proof. Assuming that $\bar{\nabla}$ is well-defined, flatness of $\bar{\nabla}$ follows directly from flatness of $\nabla$. It suffices to show that $\nabla$ restricted to $\operatorname{ker} \rho$ is zero. For this purpose, some useful properties of the anchor and $\nabla$ are derived to compute $\nabla_{\alpha}$ for $\alpha \in \Gamma(\operatorname{ker} \rho)$.

First it is shown that that the restriction of $\rho$ to $T^{*} P \otimes L$ is antisymmetric, i.e.,

$$
\zeta(\rho(\eta))=-\eta(\rho(\zeta))
$$

for any two elements $\zeta, \eta \in \Omega^{1}(P ; L)$. For $f \in C^{\infty}(P)$ and $u \in \Gamma(L)$, write $d f \otimes u=f j^{1} u-j^{1}(f u)$. The defining property (I) of the anchor shows that $L_{\rho(d f \otimes u)}(g) v=-L_{\rho(d g \otimes v)}(f) u$ for any other $g \in C^{\infty}(P)$ and any $v \in \Gamma(L)$. This shows the antisymmetry and also shows that if $\nu^{*} \subset \mathrm{T}^{*} P$ is the conormal bundle of the regular foliation $\mathcal{F}$, then $\nu^{*} \otimes L \subset \operatorname{ker} \rho$, as if $f$ is constant along the leaves of $\mathcal{F}$, then $L_{\rho(d f \otimes u)}(g) v=-L_{\rho(d g \otimes v)} u=0$.

Next, it is shown that, in fact,

$$
\nu^{*} \otimes L=\operatorname{ker} \rho \cap\left(T^{*} P \otimes L\right) .
$$

This can by checked by dimension counting: as the corank of the Jacobi manifold is $k=\operatorname{dim} \operatorname{ker} \rho$, a straightforward computation shows that the rank of $\nu^{*}$ is $k-1$. Hence, $k-1 \leq \operatorname{dim} \operatorname{ker} \rho\left(T^{*} P \otimes\right.$ $L) \leq k$, or equivalently $2 n-1 \leq \operatorname{dim} \rho\left(\mathrm{T}^{*} P \otimes L\right) \leq 2 n$. On the other hand, the antisymmetry of $\rho: T^{*} P \otimes L \rightarrow T P$ implies that the subspace $\rho\left(T^{*} P \otimes L\right) \subset \mathcal{F}$ is even dimensional. Hence, $\operatorname{dim} \rho\left(\mathrm{T}^{*} P \otimes L\right)=2 n$, and $\operatorname{dim}\left(\operatorname{ker} \rho \cap\left(T^{*} P \otimes L\right)\right)=k-1$.

A straightforward computation shows that, for any $f \in C^{\infty}(P)$ and $u, v \in \Gamma(L), \nabla_{d f \otimes u}(v)=$ $-d f\left(\rho\left(j^{1} v\right)\right) u$, hence

$$
\nabla_{\zeta}(v)=-\zeta\left(\rho\left(j^{1} v\right)\right), \quad \zeta \in \Omega^{1}(P ; L) .
$$

With the above properties in mind, write $\alpha$ as $\alpha=(u, \zeta) \in \Gamma(L) \oplus \Omega^{1}(P ; L)$. If $\alpha$ is a section of $\operatorname{ker} \rho \subset J^{1} L$, then $\nabla_{\alpha}: \Gamma(L) \rightarrow \Gamma(L)$ is $C^{\infty}(M)$-linear, hence it defines a section of $\operatorname{Hom}(L ; L)$. As $\operatorname{Hom}(L ; L)$ is trivial, there exists a function $G \in C^{\infty}(P)$ with the property that

$$
\nabla_{\alpha}(v)(p)=G(p) v(p)
$$

for any $v \in \Gamma(L)$ and any $p \in P$. With this,

$$
G(p) u(p)=\nabla_{\alpha}(u)(p)=\{u, u\}-\zeta\left(\rho\left(j^{1} u\right)\right)=\zeta(\rho(\zeta))=0,
$$

where in the third equation we use that $\rho\left(j^{1} u-\zeta\right)=0$, and in the last one the antisymmetry of $\rho$. If $u(p) \neq 0$, then $G(p)=0$, and $\nabla_{\alpha}(p)=0$; otherwise $u(p)=0, \alpha(p) \in\left(T^{*} P \otimes L\right)_{p} \cap \operatorname{ker} \rho_{p}=$ $\nu_{p}^{*} \otimes L_{p}$ and

$$
\nabla_{\alpha}(v)(p)=\alpha(p)\left(\rho\left(j_{p}^{1} v\right)\right)=0 .
$$


Lemma A.2. If $(P, L,\{\}$,$) is a regular Jacobi manifold all of whose leaves are even dimensional$ then $\operatorname{ker} \rho \subset J^{1} L$ is bundle of abelian Lie algebras.

Proof. Using the Spencer decomposition of Note 2.17, the Lie bracket of two sections $\alpha, \beta \in$ $\Gamma\left(J^{1} L\right)$ equals $(\operatorname{pr}([\alpha, \beta]), \mathrm{D}[\alpha, \beta])$. If $\alpha, \beta$ are sections of $\operatorname{ker} \rho \subset J^{1} L$, the compatibility conditions (2.4) and (2.5) imply that

$$
[\alpha, \beta]=\left(-\nabla_{\beta}(\operatorname{pr}(\alpha)), \nabla_{\alpha}(\text { D. }(\beta))-\nabla_{\beta}(D .(\alpha))\right) .
$$

The proof of Lemma A.1 shows that $\nabla$ restricted to $\operatorname{ker} \rho$ is zero, thus proving the result.

The flat connection $\bar{\nabla}$ induces the usual Koszul-like differential $\mathrm{d}_{\mathcal{F}}$ on the complex of smooth foliated forms of $\mathcal{F}$ with values in $L$. The 2 -form $\omega_{\mathcal{F}} \in \Omega^{2}(\mathcal{F} ; L)$ of equation (3.3) defines a canonical cohomology class in $\mathrm{H}^{2}(\mathcal{F} ; L)$, as the next lemma shows.

Lemma A.3. Under the hypotheses of Lemma A.1, $\omega_{\mathcal{F}}$ is well defined, and $\mathrm{d}_{\mathcal{F}} \omega_{\mathcal{F}}=0$.

Proof. Since sections of the form $j^{1} u$, for $u \in \Gamma(L)$ form a $C^{\infty}(P)$-basis of $\Gamma\left(J^{1} L\right)$ and $T \mathcal{F}=$ $\rho\left(J^{1} L\right)$, equation (3.3) defines a unique map $\Gamma(T \mathcal{F}) \times \Gamma(T \mathcal{F}) \rightarrow \Gamma(L)$ which is manifestly antisymmetric. Let $f \in C^{\infty}(P)$ and fix $u, v \in \Gamma(L)$; then

$$
\begin{aligned}
\omega_{\mathcal{F}}\left(f \rho\left(j^{1} u\right), \rho\left(j^{1} v\right)\right) & =\omega_{\mathcal{F}}\left(\rho(f u, \mathrm{~d} f \otimes u), \rho\left(j^{1} v\right)\right)=\{f u, v\}+\mathrm{d} f \otimes u\left(\rho\left(j^{1} v\right)\right) \\
& =f\{u, v\}-\mathcal{L}_{\rho\left(j^{1} v\right)} f u+\mathcal{L}_{\rho\left(j^{1} v\right)} f u=f \omega_{\mathcal{F}}\left(\rho\left(j^{1} u\right), \rho\left(j^{1} v\right)\right),
\end{aligned}
$$

where the first equality follows from the $C^{\infty}(P)$-structure on $\Gamma\left(J^{1} L\right)$ arising from the Spencer decomposition (cf. Note 2.17), the second by definition of $\omega_{\mathcal{F}}$, and the third by the characterising property (I) of the anchor $\rho$ (cf. Proposition 2.11). The above calculation shows that equation (3.3) indeed defines a foliated 2 -form.

To check that $\mathrm{d}_{\mathcal{F}} \omega_{\mathcal{F}}=0$ it suffices to check that

$$
\mathrm{d}_{\mathcal{F}} \omega_{\mathcal{F}}\left(\rho\left(j^{1} u\right), \rho\left(j^{1} v\right), \rho\left(j^{1} w\right)\right)=0
$$

for any $u, v, w \in \Gamma(L)$. This is because $\mathrm{d}_{\mathcal{F}} \omega_{\mathcal{F}}$ is $C^{\infty}(P)$-linear in each entry and sections of the form $\rho\left(j^{1} u\right)$ form a $C^{\infty}(P)$-basis of $\Gamma(T \mathcal{F})$. Then

$$
\begin{aligned}
\mathrm{d}_{\mathcal{F}} \omega_{\mathcal{F}} & \left(\rho\left(j^{1} u\right), \rho\left(j^{1} v\right), \rho\left(j^{1} w\right)\right) \\
& =\bar{\nabla}_{\rho\left(j^{1} u\right)}\left(\omega_{\mathcal{F}}\left(\rho\left(j^{1} v\right), \rho\left(j^{1} w\right)\right)\right)+\text { c.p. }-\left(\omega_{\mathcal{F}}\left(\left[\rho\left(j^{1} u\right), \rho\left(j^{1} v\right)\right], \rho\left(j^{1} w\right)\right)+\text { c.p. }\right) \\
& =\{u,\{v, w\}\}+\text { c.p. }-\left(\omega_{\mathcal{F}}\left(\rho\left(\left[j^{1} u, j^{1} v\right]\right), \rho\left(j^{1} w\right)\right)+\text { c.p. }\right) \\
& =-\left(\omega_{\mathcal{F}}\left(\rho\left(j^{1}\{u, v\}\right), \rho\left(j^{1} w\right)\right)+\text { c.p. }\right)=-(\{\{u, v\}, w\}+\text { c.p. })=0,
\end{aligned}
$$

where c.p. stands for cyclic permutation, the second equality follows from the fact that $\rho$ is a map of Lie algebroids, the third by the defining property (II) of the Lie bracket on $J^{1} L$ (cf. Proposition 2.11) and by the Jacobi identity for $\{\cdot, \cdot\}$, which also implies the last equality. This shows that $\mathrm{d}_{\mathcal{F}} \omega_{\mathcal{F}}=0$, as required.

Using the above ideas, we can prove Note 4.20 .

Proof of Note 4.20. Since $\Gamma(T \mathcal{F})$ is generated by elements of the form $\rho\left(j^{1} u\right)$ for $u \in \Gamma\left(J^{1} L\right)$, it suffices to check that $\mathrm{D}_{\rho\left(j^{1} u\right)}(\alpha)=\mathrm{d}_{\mathcal{F}}(\operatorname{pr}(\alpha))\left(\rho\left(j^{1} u\right)\right)$ for any $u \in \Gamma\left(J^{1} L\right)$. Fix such a section. Then

$$
\begin{aligned}
\mathrm{D}_{\rho\left(j^{1} u\right)}(\alpha) & =\nabla_{\alpha} u+\operatorname{pr}\left(\left[j^{1} u, \alpha\right]\right)=\operatorname{pr}\left(\left[j^{1} u, \alpha\right]\right)=-\operatorname{pr}\left(\left[\alpha, j^{1} u\right]\right) \\
& =\nabla_{j^{1} u}(\operatorname{pr}(\alpha))-\mathrm{D}_{\rho(\alpha)}\left(j^{1} u\right)=\{u, \operatorname{pr}(\alpha)\}=\mathrm{d}_{\mathcal{F}}(\operatorname{pr}(\alpha))\left(\rho\left(j^{1} u\right)\right),
\end{aligned}
$$

where the first and fourth equalities use the compatibility condition (2.4) for the Spencer operator, the second uses that $\alpha \in \Gamma(\operatorname{ker} \rho)$, the third exploits anti-symmetry of the Lie bracket, and the last two follow by definition of $\nabla$ (cf. equation (2.3)). 


\section{B Proofs of the main results of Section 2.3}

Proof of Proposition 2.25. Suppose that the statement does not hold. Then there exists $\alpha \in L^{*} \backslash\{0\} \subset T^{*} M$ with $\mu(\alpha)=0$. Recall that $\mu$ is the restriction of the moment map of the cotangent lift which preserves the Liouville 1-form on $T^{*} M$ (cf. Note 2.19); unravelling the definitions, it follows that, for any $\xi \in \mathfrak{g}$,

$$
\langle\mu(\alpha), \xi\rangle=\alpha\left(D_{\alpha} \operatorname{pr}\left(\xi_{L^{*} \backslash\{0\}}(\alpha)\right)\right)
$$

where $\xi_{L^{*} \backslash\{0\}}(\alpha)$ is the image of $\xi$ under the infinitesimal action of $G$ on $L^{*} \backslash\{0\}$ evaluated at $\alpha$. Since pr: $L^{*} \backslash\{0\} \rightarrow M$ is $G$-equivariant,

$$
D_{\alpha} \operatorname{pr}\left(\xi_{L^{*} \backslash\{0\}}(\alpha)\right)=\xi_{M}(p),
$$

where $p=\operatorname{pr}(\alpha)$. Thus, for any $\xi \in \mathfrak{g}, \alpha\left(\xi_{M}(p)\right)=0$, which implies that $\xi_{M}(p) \in H_{p}$, in turn yielding that $T_{p}(G \cdot p) \subset H_{p}$.

Let $\theta$ be a contact 1 -form defined on a $G$-invariant neighbourhood $U$ of $p$, i.e., $H=\operatorname{ker} \theta$ locally and $\left.\mathrm{d} \theta\right|_{H}$ is symplectic. By [25, Lemma 2.6], it may be assumed that $\theta$ is $G$-invariant. For any $\xi, \xi^{\prime} \in \mathfrak{g}$,

$$
\mathrm{d} \theta_{p}\left(\xi_{M}(p), \xi_{M}^{\prime}(p)\right)=\theta_{p}\left(\left[\xi_{M}, \xi_{M}^{\prime}\right](p)\right)=\theta_{p}\left(\left[\xi, \xi^{\prime}\right]_{M}(p)\right)=0,
$$

where the first and third equalities follow from the fact that $T_{p}(G \cdot p) \subset H_{p}$. Therefore $T_{p}(G \cdot p)$ is an isotropic subspace of $\left(H_{p}, \mathrm{~d} \theta_{p}\right)$.

The 1-form $\theta$ determines a trivialisation of $\left.L^{*}\right|_{U} \cong U \times \mathbb{R}$. Henceforth, identify any $\left.\alpha^{\prime} \in L^{*}\right|_{U}$ with the pair $\left(p^{\prime}, t^{\prime}\right) \in U \times \mathbb{R}$, where $\operatorname{pr}\left(\alpha^{\prime}\right)=p^{\prime}$ and $\alpha^{\prime}=t^{\prime} \theta_{p}$. Since $\theta$ is $G$-invariant, the induced $G$-action on $\left.L^{*}\right|_{U}$ is given in this trivialisation by

$$
g \cdot\left(p^{\prime}, t^{\prime}\right)=\left(g \cdot p^{\prime}, t^{\prime}\right)
$$

Setting $\alpha=(p, t)$ (where $t \neq 0), G$-invariance of $\theta$ implies that there is a splitting as $G_{\alpha}=G_{p^{-}}$ symplectic vector spaces

$$
\left(T_{\alpha}\left(L^{*} \backslash\{0\}\right), \Omega_{\alpha}\right)=\left(H_{p}, t \mathrm{~d} \theta_{p}\right) \oplus\left(\mathbb{R}\left\langle R_{p}, \frac{\partial}{\partial t}\right\rangle, \mathrm{d} t \wedge \theta_{p}\right)=:\left(H_{p}, t \mathrm{~d} \theta_{p}\right) \oplus\left(V, \omega_{V}\right),
$$

where $R$ is the locally defined Reeb vector field associated to the contact 1 -form $\theta$, i.e., $\theta(R)=1$ and $\mathrm{d} \theta(R,-)=0$, and the $G_{\alpha}$-action on $V$ is trivial. Moreover,

$$
T_{\alpha}(G \cdot \alpha)=T_{(p, t)}(G \cdot(p, t))=T_{p}(G \cdot p) \oplus 0 ;
$$

since $T_{p}(G \cdot p) \subset\left(H_{p}, \mathrm{~d} \theta_{p}\right)$ is isotropic, equation (B.1) implies that $T_{\alpha}(G \cdot \alpha) \subset\left(T_{\alpha}\left(L^{*} \backslash\{0\}\right), \Omega_{\alpha}\right)$ is isotropic. In fact,

$$
\left(T_{\alpha}(G \cdot \alpha)\right)^{\Omega_{\alpha}}=\left(T_{p}(G \cdot p)\right)^{\mathrm{d} \theta_{p}} \oplus V .
$$

Setting

$$
N_{\alpha}:=\frac{\left(T_{\alpha}(G \cdot \alpha)\right)^{\Omega_{\alpha}}}{T_{\alpha}(G \cdot \alpha)} \quad \text { and } \quad N_{p}:=\frac{\left(T_{p}(G \cdot p)\right)^{\mathrm{d} \theta_{p}}}{T_{p}(G \cdot p)},
$$

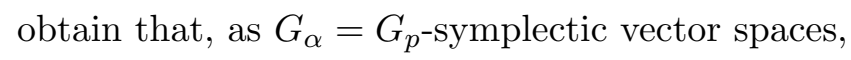

$$
\left(N_{\alpha}, \omega_{N_{\alpha}}\right) \cong\left(N_{p}, \omega_{N_{p}}\right) \oplus(V, \omega),
$$


where the $G_{p}$-action on the right hand side is the product of the induced one on the first factor and the trivial one on the second.

Since $G$ is compact, the Marle-Guillemin-Sternberg local normal form for Hamiltonian actions holds (cf. [20,27]); as $T_{\alpha}(G \cdot \alpha)$ is isotropic, this reduces to the following. Set $\mu(\alpha)=\eta \in \mathfrak{g}^{*}$; $G$-equivariance of $\mu$ gives the following inclusion of stabilisers $G_{\alpha} \subset G_{\eta}$, where $G \curvearrowright \mathfrak{g}^{*}$ acts by the coadjoint action. This induces an analogous inclusion for the corresponding Lie algebras $\mathfrak{g}_{\alpha} \subset \mathfrak{g}_{\eta}$. Fix a $G_{\alpha}$-invariant inner product on $\mathfrak{g}$, which induces a $G_{\alpha}$-invariant orthogonal direct sum decomposition $\mathfrak{g}_{\eta}=\mathfrak{g}_{\alpha} \oplus \mathfrak{m}$. Then a $G$-invariant neighbourhood of $G \cdot \alpha$ in $L^{*} \backslash\{0\}$ is isomorphic, as a Hamiltonian $G$-space, to a $G$-invariant neighbourhood of $G \cdot[e, 0,0]$ in

$$
\left(Y, \omega_{Y}\right):=\left(G \times{ }_{G_{\alpha}}\left(\mathfrak{m}^{*} \times N_{\alpha}\right), \omega_{Y}\right),
$$

where the right hand side is, as a smooth manifold, the quotient of $G \times\left(\mathfrak{m}^{*} \times N_{\alpha}\right)$ by the antidiagonal action of $G_{\alpha}$, and the action of $G$ on $Y$ descends from the left action of $G$ on the first factor of $G \times\left(\mathfrak{m}^{*} \times N_{\alpha}\right)$. Following [29, 30], the closed 2-form $\omega_{Y}$ (which is symplectic only near $G \cdot[e, 0,0])$ can be constructed using Marsden-Weinstein reduction on the presymplectic manifold $\left(G \times \mathfrak{g}_{\eta}^{*} \times N_{\alpha}, \sigma \oplus \omega_{\alpha}\right)$ endowed with the induced Hamiltonian $G_{\alpha}$-action, where $\sigma$ is a closed 2-form on $G \times \mathfrak{g}_{\eta}^{*}$ constructed as in [29]. By the splitting of equation (B.2), it follows that, as Hamiltonian a $G_{\alpha}$-space, $\left(G \times \mathfrak{g}_{\eta}^{*} \times N_{\alpha}, \sigma \oplus \omega_{\alpha}\right)$ equals

$$
\left(G \times \mathfrak{g}_{\eta}^{*} \times N_{p} \times V, \sigma \oplus \omega_{\alpha} \oplus \omega_{V}\right),
$$

where the $G_{\alpha}$-action on the last factor is trivial. Unravelling the construction of in [29, 30], it follows that, as a presymplectic Hamiltonian $G$-space,

$$
\left(Y, \omega_{Y}\right) \cong\left(W \times V, \omega_{W} \oplus \omega_{V}\right):=\left(\left(G \times_{G_{\alpha}}\left(\mathfrak{m}^{*} \times N_{p}\right)\right) \times V, \omega_{W} \oplus \omega_{V}\right),
$$

where the $G$-action on $V$ is trivial; this also implies that the $G$-action on the presymplectic manifold $\left(W, \omega_{W}\right)$ is Hamiltonian. Restricting to a $G$-invariant neighbourhood of the image of $G \cdot \alpha$ on which $\omega_{W} \oplus \omega_{V}$ is symplectic, the Hamiltonian action of $G$ is multiplicity-free; it follows, in particular, that the action of $G$ on $W$ is locally free at some point $w \in W$; by the principal orbit theorem (cf. [14, Theorem 2.8.5]), there is an open dense set of $W$ whose stabiliser is discrete. Seeing as $\left(W, \omega_{W}\right)$ is symplectic on a $G$-invariant neighbourhood $U$ of $G \cdot[e, 0,0]$, it follows that the Hamiltonian $G$-action on $\left(U,\left.\omega_{W}\right|_{U}\right)$ is locally free at some point in $U$, which implies that $\operatorname{dim} U=\operatorname{dim} W \geq \operatorname{dim} G+\operatorname{rk} G$ by [19, Theorem 5.1.6]. However, $\operatorname{dim} W=\operatorname{dim} Y-\operatorname{dim} V=\operatorname{dim} L^{*} \backslash\{0\}-2$; this contradicts the fact that the action of $G$ on $(M, H)$ is multiplicity-free, thus completing the proof.

Proof of Theorem 2.29. First it is shown that $F_{\phi}: \phi^{*}(O(1)) \rightarrow L$ as defined in the statement is, in fact, a vector bundle isomorphism. Consider $H_{\phi}: L \rightarrow \phi^{*}(O(1))$ defined by

$$
u \in L_{p} \mapsto\left(p, \eta_{u}\right),
$$

where $\eta_{u}$ is defined as follows. Let $\alpha \in L_{p}^{*} \backslash\{0\}$; then $\eta_{u}: \mathbb{R}\left\langle\mu_{u}(\alpha)\right\rangle \rightarrow \mathbb{R}$ is given by $t \mu_{u}(\alpha) \mapsto$ $t\langle\alpha, u\rangle$. It is straightforward to check that the definition of $\eta_{u}$ does not depend on the choice of $\alpha \in L_{p}^{*} \backslash\{0\}$. Smoothness of $H_{\phi}$ can be checked by writing the map explicitly once a local trivialisation of pr: $L^{*} \backslash\{0\} \rightarrow M$ is fixed. Fibrewise linearity of $H_{\phi}$ follows immediately from the definition, while its fibrewise injectivity follows from the fact that $\alpha \in L_{p}^{*} \backslash\{0\}$. Seeing as $H_{\phi}$ is a map between line bundles, it follows that it is an isomorphism. The map $F_{\phi}$ is easily checked to be its inverse, therefore proving that it is a vector bundle isomorphism. Checking that $\phi$ is Jacobi with bundle component $F_{\phi}$ can be done as follows. Unravelling the various definitions, obtain that, for all $s \in \Gamma(O(1))$, following equality of maps on $O(1)$,

$$
F_{\mathrm{pr}} \circ \mathrm{pr}^{*} \circ F_{\phi} \circ \phi^{*}(s)=\mu^{*} \circ F_{\pi} \circ \pi^{*}(s)
$$


where $\pi: \mathfrak{g}^{*} \backslash\{0\} \rightarrow \mathbb{P}\left(\mathfrak{g}^{*}\right)$ is the projection, and $F_{\mathrm{pr}}: \operatorname{pr}^{*} L \rightarrow \mathbb{R}_{L^{*} \backslash\{0\}}$ and $F_{\pi}: \pi^{*}(O(1)) \rightarrow$ $\mathbb{R}_{\mathfrak{g}^{*} \backslash\{0\}}$ are the isomorphisms defined in Examples 2.9 and 2.10 respectively. Since $\mu$ is Jacobi and $\pi$ is Jacobi with bundle component $F_{\pi}$, it follows that, for any $s_{1}, s_{2} \in \Gamma(O(1))$,

$$
\mu^{*} \circ F_{\pi} \circ \pi^{*}\left\{s_{1}, s_{2}\right\}=\left\{\mu^{*} \circ F_{\pi} \circ \pi^{*}\left(s_{1}\right), \mu^{*} \circ F_{\pi} \circ \pi^{*}\left(s_{2}\right)\right\} ;
$$

equation (B.3) implies that

$$
\begin{aligned}
F_{\mathrm{pr}} \circ \operatorname{pr}^{*} \circ F_{\phi} \circ \phi^{*}\left\{s_{1}, s_{2}\right\} & =\left\{F_{\mathrm{pr}} \circ \operatorname{pr}^{*} \circ F_{\phi} \circ \phi^{*}\left(s_{1}\right), F_{\mathrm{pr}} \circ \operatorname{pr}^{*} \circ F_{\phi} \circ \phi^{*}\left(s_{2}\right)\right\} \\
& =F_{\mathrm{pr}} \circ \operatorname{pr}^{*}\left\{F_{\phi} \circ \phi^{*}\left(s_{1}\right), F_{\phi} \circ \phi^{*}\left(s_{2}\right)\right\},
\end{aligned}
$$

where the last equality follows from the fact that pr is Jacobi with bundle component $F_{\mathrm{pr}}$. Equation (B.4) implies that $\phi$ is Jacobi with bundle component $F_{\phi}$ by noticing that $F_{\mathrm{pr}}$ is an isomorphism and that $\mathrm{pr}^{*}$ is injective.

Henceforth, fix a point $p \in M_{\text {prin }}$. Since pr: $L^{*} \backslash\{0\} \rightarrow M$ is $G$-equivariant, the stabiliser of any $\alpha \in L_{p}^{*} \backslash\{0\}$ is also discrete. Fix such an $\alpha$. By [19, Corollary 5.1.2], $D_{\alpha} \mu$ is surjective; since $\pi \circ \mu=\phi \circ$ pr and $\pi$ is a submersion, property (J1) follows. Suppose that property (J2) does not hold, then, since $H \subset T M$ has codimension 1, it follows that ker $D_{p} \phi \subset H_{p}$. First, observe that $D_{\alpha}$ pr restricted to $\operatorname{ker} D_{\alpha} \mu$ is an isomorphism onto $\operatorname{ker} D_{p} \phi$ : one inclusion follows from $\pi \circ \mu=\phi \circ \mathrm{pr}$, and the other by dimension counting and the fact that property (J1) holds. In fact, since pr: $L^{*} \backslash\{0\} \rightarrow M$ is an $\mathbb{R}^{*}$-principal bundle, write

$$
T_{\alpha} L^{*} \backslash\{0\}=T_{p} M \oplus \mathbb{R}\left\langle\frac{\partial}{\partial t}\right\rangle=H_{p} \oplus \mathbb{R}\left\langle R_{p}\right\rangle \oplus \mathbb{R}\left\langle\frac{\partial}{\partial t}\right\rangle,
$$

where $\alpha\left(R_{p}\right)=1$ and $\mathrm{d} \alpha\left(R_{p},-\right)=0$, and $D_{\alpha}$ pr is simply projection onto the first two components. Since by assumption ker $D_{p} \phi \subset H_{p}$, it follows that ker $D_{\alpha} \mu \cap 0 \oplus \mathbb{R}\left\langle R_{p}\right\rangle \oplus 0=\{0\}$. Since ker $D_{\alpha} \mu=\left(T_{\alpha}(G \cdot \alpha)\right)^{\Omega}$ by [19, Corollary 5.1.4], it follows that $\mathbb{R}\left\langle\frac{\partial}{\partial t}\right\rangle \subset T_{\alpha}(G \cdot \alpha)$, which is a contradiction, since $\operatorname{dim} G \cdot \alpha=\operatorname{dim} G \cdot p$ as both stabiliser subgroups are discrete. Therefore (J2) holds.

It remains to prove condition (J3). First, it is shown that $\operatorname{ker} D_{\alpha} \mu$ is isotropic. Since $\mu$ is $G$ equivariant, the range of $D_{\alpha} \mu$ restricted to $T_{\alpha}(G \cdot \alpha)$ is $T_{\mu(\alpha)}(G \cdot \mu(\alpha))$. The dimension of the latter is at most $\operatorname{dim} G-\operatorname{rk} G$, while the dimension of $D_{\alpha} \mu\left(T_{\alpha}(G \cdot \alpha)\right)$ is at most $\operatorname{dim} G-\operatorname{dim}\left(\operatorname{ker} D_{\alpha} \mu\right)=$ $\operatorname{dim} G-\left(\operatorname{dim}\left(L^{*} \backslash\{0\}\right)-\operatorname{dim} G\right)=\operatorname{dim} G-\operatorname{rk} G$, where the first equality follows from the fact that $D_{\alpha} \mu$ is surjective, and the second from definition of multiplicity-free action. Therefore ker $D_{\alpha} \mu=\left(T_{\alpha}(G \cdot \alpha)\right)^{\Omega} \subset T_{\alpha}(G \cdot \alpha)$, which proves that ker $D_{\alpha} \mu$ is isotropic. Using the fact that $D_{\alpha} \mu$ is surjective, this condition is equivalent to the following inclusion (suppressing the dependence on $\alpha$ to simplify notation)

$$
\text { ker } D \mu \subset \rho_{L^{*} \backslash\{0\}}\left(\mu^{*} J^{1}\left(\mathfrak{g}^{*} \backslash\{0\}\right)\right),
$$

where $\rho_{L^{*} \backslash\{0\}}$ is the anchor of the Lie algebroid associated to the Jacobi manifold $\left(L^{*} \backslash\{0\}, \Omega\right)$. In fact, since $\left(L^{*} \backslash\{0\}, \Omega\right)$ is Poisson, the right hand side equals $\rho_{L^{*} \backslash\{0\}}\left(\mu^{*}\left(T^{*}\left(\mathfrak{g}^{*} \backslash\{0\}\right) \oplus 0\right)\right)$, where $J^{1}\left(\mathfrak{g}^{*} \backslash\{0\}\right)=T^{*}\left(\mathfrak{g}^{*} \backslash\{0\}\right) \oplus \mathbb{R}$ (cf. Note 2.12). Unravelling the definitions, it can be checked that the map $F_{\pi} \circ \pi^{*}: O(1) \rightarrow J^{1}\left(\mathfrak{g}^{*} \backslash\{0\}\right)$ is transverse to $0 \oplus \mathbb{R}$. Therefore, equation (B.5) can be written as

$$
\operatorname{ker} D \mu \subset \rho_{L^{*} \backslash\{0\}}\left(\mu^{*} \circ F_{\pi} \circ \pi^{*}(O(1))\right)=\rho_{L^{*} \backslash\{0\}}\left(F_{\mathrm{pr}} \circ \mathrm{pr}^{*} \circ F_{\phi} \circ \phi^{*}(O(1))\right),
$$

where the last equality follows from equation (B.3). Applying $D_{\alpha}$ pr to both sides and observing that $D_{\alpha} \operatorname{pr}\left(\operatorname{ker} D_{\alpha} \mu\right)=\operatorname{ker} D_{p} \phi$, have that

$$
\text { ker } D \phi \subset D \operatorname{pr} \circ \rho_{L^{*} \backslash\{0\}}\left(F_{\text {pr }} \circ \operatorname{pr}^{*} \circ F_{\phi} \circ \phi^{*}(O(1))\right) \text {. }
$$

Since pr is Jacobi with bundle component $F_{\text {pr }}$, the right hand side equals $\rho\left(F_{\phi}\left(\phi^{*}(O(1))\right)\right)$ - cf. Note 2.13 - which equals $\left(\operatorname{ker} D_{p} \phi\right)^{\perp}$ by definition. Thus property (J3) holds. 


\section{C $\quad$ Missing proofs from Sections 4.2 and 4.3}

\section{Proofs of results of Section 4.2}

The proof of Lemma 4.9 and the associated preliminary results use in a crucial fashion the geometry of the Spencer operator associated to $(P, L,\{\cdot, \cdot\})$. Fix a CIR $\phi:(M, H) \rightarrow(P, L,\{\}$, and let $\theta$ denote the generalised contact form associated to $(M, H)$. Recall that for $\alpha \in \Gamma(\operatorname{ker} \rho)$, $\psi\left(\phi^{*} \alpha\right) \in \Gamma(\operatorname{ker} D \phi)$, where $\psi: \phi^{*} \operatorname{ker} \rho \rightarrow T M$ is defined as in Lemma 4.2. Therefore, the flow $\varphi_{\alpha}^{t}: M \rightarrow M$ of $\psi\left(\phi^{*} \alpha\right)$ preserves the fibres of $\phi$, i.e., $\phi \circ \varphi_{\alpha}^{t}=\phi$ for all $t \in \mathbb{R}$. Hence, $\left(\varphi_{\alpha}^{t}\right)^{*} \phi^{*} L \cong$ $\phi^{*} L$ canonically. This allows to define a 'Lie derivative' type operator on $\Omega^{*}\left(M ; \phi^{*} L\right)$ by

$$
\left(\mathcal{L}_{\alpha} \omega\right)_{m}=\left.\frac{d}{d t}\right|_{t=0}\left(\left(\varphi_{\alpha}^{t}\right)^{*} \omega\right)_{m}
$$

for any $m \in M$ and $\omega \in \Omega^{l}\left(M, \phi^{*} L\right)$. It obeys rules which are analogous to those of the standard Lie derivative, for instance,

$$
\left.\frac{d}{d t}\right|_{t=s}\left(\left(\varphi_{\alpha}^{t}\right)^{*} \omega\right)_{m}=\left(\left(\varphi_{\alpha}^{s}\right)^{*}\left(\mathcal{L}_{\alpha} \omega\right)\right)_{m}
$$

as well as the following property proved in [8, Lemma 3.8].

Lemma C.1. For any $\omega \in \Omega^{l}\left(M ; \phi^{*} L\right)$ and $X \in \mathfrak{X}(M)$,

$$
\left[i_{X}, \mathcal{L}_{\alpha}\right] \omega=i_{\left[X, \psi\left(\phi^{*} \alpha\right)\right]} \omega .
$$

With the above preliminary results, the following can be proved.

Lemma C.2. For any $\alpha \in \Gamma(\operatorname{ker} \rho)$,

$$
\left(\varphi_{(u, \eta)}^{1}\right)^{*} \theta-\theta=\phi^{*} \eta
$$

where $\alpha=(u, \eta) \in \Gamma(L) \oplus \Omega^{1}(P ; L)$ in the Spencer decomposition.

Proof. Let $(u, \eta) \in \Gamma(\operatorname{ker} \rho)$ and $X \in \mathfrak{X}(M)$. It suffices to show that

$$
\left(\frac{\mathrm{d}}{\mathrm{d} t}\left(\left(\varphi_{(u, \eta)}^{t}\right)^{*} \theta\right)\right)(X)=\left(\phi^{*} \eta\right)(X) ;
$$

for the lemma follows by integrating from $t=0$ to $t=1$. Computing the left hand side of equation (C.3) using equation (C.2) and Lemma C.1, obtain that

$$
\left(\frac{\mathrm{d}}{\mathrm{d} t}\left(\left(\varphi_{(u, \eta)}^{t}\right)^{*} \theta\right)\right)(X)=\mathcal{L}_{(u, \eta)}\left(\theta\left(D \varphi_{(u, \eta)}^{t}(X)\right)\right)+\theta\left[D \varphi_{(u, \eta)}^{t}(X), \psi\left(\phi^{*} u, \phi^{*} \eta\right)\right] .
$$

Set

$$
D \varphi_{(u, \eta)}^{t}(X)=\sum_{i} f_{t, i} A_{t, i}+X_{t}
$$

where $f_{t, i} \in C^{\infty}(M), A_{t, i}:=\psi\left(\phi^{*} u_{t, i}, \phi^{*} \eta_{t, i}\right) \in \Gamma(\operatorname{ker} D \phi)$ with $\left(u_{t, i}, \eta_{t, i}\right) \in \Gamma(\operatorname{ker} \rho)$, and $X_{t} \in \Gamma(H)$ are all time-dependent, with $D \phi\left(X_{t}\right)=D \phi(X)$. This decomposition follows from property (CR2) and the fact that the action $\psi: \Gamma\left(\phi^{*} \operatorname{ker} \rho\right) \rightarrow \Gamma(\operatorname{ker} D \phi)$ is an isomorphism. Without loss of generality, assume $i=1$. On the one hand,

$$
\theta\left(D \varphi_{(u, \eta)}^{t}(X)\right)=\theta\left(f_{t} \psi\left(\phi^{*} u_{t}, \phi^{*} \eta_{t}\right)\right)=f_{t} \theta\left(\rho_{M}\left(\phi^{*} u_{t}, \phi^{*} \eta_{t}\right)\right)=f_{t} \phi^{*} u_{t}
$$


since the anchor $\rho_{M}$ of a contact manifold can be easily computed to be $\rho_{M}\left(\phi^{*} u_{t}, \phi^{*} \eta_{t}\right)=$ $R_{\phi^{*} u_{t}}+c^{\sharp}\left(\left.\phi^{*} \eta_{t}\right|_{H}\right)$. By definition of $c^{\sharp}$ and of Reeb vector fields, $c^{\sharp}\left(\left.\phi^{*} \eta_{t}\right|_{H}\right) \in \Gamma(H)$ and $\theta\left(R_{\phi^{*} u_{t}}\right)=\phi^{*} u_{t}$ for all $t$ (cf. Example 2.5). Using equation (C.6) and the fact that $\phi \circ \varphi_{(u, v)}^{s}=\phi$, have that $\phi^{*} u_{t, \varphi_{(u, v)}^{s}(m)}=u_{t, \phi(m)}$ for all $s$; this in turn implies that

$$
\mathcal{L}_{(u, \eta)}\left(\theta\left(D \varphi_{(u, \eta)}^{t}(X)\right)\right)_{m}=\mathcal{L}_{\psi\left(\phi^{*} u, \phi^{*} \eta\right)}\left(f_{t}\right)_{m} u_{t, m}
$$

On the other hand, using equation (C.5)

$$
\begin{aligned}
\theta\left(\left[D \varphi_{(u, \eta)}^{t}(X), \psi\left(\phi^{*} u, \phi^{*} \eta\right)\right]\right) & =\theta\left(\left[X_{t}, \psi\left(\phi^{*} u, \phi^{*} \eta\right)\right]\right)-\mathcal{L}_{\psi\left(\phi^{*} u, \phi^{*} \eta\right)}\left(f_{t}\right) \theta\left(A_{t}\right) \\
& =\theta\left(\left[X_{t}, \psi\left(\phi^{*} u, \phi^{*} \eta\right)\right]\right)-\mathcal{L}_{\psi\left(\phi^{*} u, \phi^{*} \eta\right)}\left(f_{t}\right) \phi^{*} u_{t}
\end{aligned}
$$

where the first equality follows from the fact that $A_{t}, \psi\left(\phi^{*} u, \phi^{*} \eta\right) \in \Gamma(\operatorname{ker} D \phi)$ and this is a bundle of abelian Lie algebras, and the second by definition of $\theta\left(A_{t}\right)$. Plugging equations (C.7) and (C.8) into equation (C.4), obtain that

$$
\left(\frac{\mathrm{d}}{\mathrm{d} t}\left(\varphi_{(u, \eta)}^{t}\right)^{*} \theta\right)(X)=\left(\varphi_{(u, \eta)}^{t}\right)^{*}\left(\theta\left(\left[X_{t}, \psi\left(\phi^{*} u, \phi^{*} \eta\right)\right]\right)\right)
$$

the right hand side of equation (C.9) can be computed to be

$$
\left.\theta\left(\left[X_{t}, c^{\sharp}\left(\left.\phi^{*} \eta\right|_{H}\right)\right]\right)=\left.\phi^{*} \eta\right|_{H}\left(X_{t}\right)=\phi^{*}\left(\eta\left(D \phi\left(X_{t}\right)\right)\right)\right)=\phi^{*}(\eta(D \phi(X)),
$$

where the last equality uses that $D \phi\left(X_{t}\right)=D \phi(X)$. Therefore, equation (C.9) becomes

$$
\left(\frac{\mathrm{d}}{\mathrm{d} t}\left(\varphi_{(u, \eta)}^{t}\right)^{*} \theta\right)(X)=\left(\varphi_{(u, \eta)}^{t}\right)^{*} \phi^{*}(\eta D \phi(X))=\phi^{*}(\eta(D \phi X))=\left(\phi^{*} \eta\right)(X)
$$

where the second equality follows from $\phi \circ \varphi_{(u, \eta)}^{t}=\phi$.

Lemma 4.9 is a obtained as a corollary of Lemma C.2.

Proof of Lemma 4.9. Let $\phi:(M, H) \rightarrow(P, L,\{\cdot, \cdot\})$ be a CIR whose period net is $\Sigma$. Use the Spencer decomposition to write a section as $(u, \eta) \in \Gamma(\Sigma)$ (possibly locally defined). As $\Sigma$ is the isotropy of the action defined by $(4.3), \varphi_{(u, \eta)}^{1}=\mathrm{id}$. Applying Lemma C.2 to the section $(u, \eta)$, obtain that $\phi^{*} \eta=0$, hence $\eta=0$. This means precisely that $(u, \eta)$ is holonomic, i.e., of the form $j^{1} u$, as required.

Having established properties of the above geometric properties of the period bundle of a CIR, we turn to the proof of the result relating transversal $\mathbb{Z}$-projective structures with transversal $\mathbb{Z}$-projective lattices.

Proof of Proposition 4.14. Throughout this proof, fix a foliated manifold $(N, \mathcal{F})$, where $\mathcal{F}$ has codimension $l$. The proof first shows how to construct a transversal $\mathbb{Z}$-projective structure starting from a lattice and then the opposite construction is described. Checking that the constructions are inverse to one another is left as an exercise for the reader.

From lattices to structures. Fix a line bundle $\pi: L \rightarrow N$ and a transversal $\mathbb{Z}$-projective lattice $\Sigma \subset J^{1} L$. Choose an open cover $\mathcal{U}=\left\{U_{i}\right\}$ of $N$ with the following properties

- there exists a nowhere vanishing section $z_{i}: U_{i} \rightarrow \pi^{-1}\left(U_{i}\right)$;

- there exists a trivialisation of $\left.\Sigma\right|_{U_{i}}$ with local frame $\alpha_{1}^{i}, \ldots, \alpha_{l+1}^{i} \in \Gamma\left(\left.\Sigma\right|_{U_{i}}\right)$. 
Fix the above trivialisations and let $c_{i j}: U_{i j} \rightarrow \mathrm{GL}(1 ; \mathbb{R})$ and $A_{i j}: U_{i j} \rightarrow \mathrm{GL}(l+1 ; \mathbb{Z})$ denote the corresponding transition functions. In fact, without loss of generality, it may be assumed that, for all $i, j, c_{i j}$ takes values in $\mathrm{GL}(1 ; \mathbb{Z})=\mathrm{O}(1 ; \mathbb{R})$. Property (T3) implies that for all $i$ and each $r=1, \ldots, l+1$, there exist smooth functions

$$
g_{r}^{i}: \quad U_{i} \rightarrow \mathbb{R} \quad \text { with } \quad \alpha_{r}^{i}=j^{1}\left(g_{r}^{i} z_{i}\right) .
$$

The transversal $\mathbb{Z}$-projective structure is constructed using these functions $g_{r}^{i}$; before proceeding to the construction, two preparatory claims are proved.

Claim C.3. The map $\bar{\chi}_{i}: U_{i} \rightarrow \mathbb{R}^{l+1}, \bar{\chi}_{i}:=\left(g_{1}^{i}, \ldots, g_{l+1}^{i}\right)$, takes values in $\mathbb{R}^{l+1} \backslash 0$ and is transversal to the Euler vector field $E=\sum_{r} x_{r} \frac{\partial}{\partial x_{r}}$.

Proof of Claim C.3. As $j^{1}\left(g_{1}^{i} z_{i}\right), \ldots, j^{1}\left(g_{l+1}^{i} z_{i}\right)$ are a frame of $\left.\Sigma\right|_{U_{i}}$ and $\Sigma^{\mathbb{R}} \rightarrow L$ is onto by property $(\mathrm{T} 2)$, it follows that $\bar{\chi}_{i} \neq 0$. Secondly, to prove that $\bar{\chi}_{i}$ is transversal to $E$, i.e., $\operatorname{Im} D \bar{\chi}_{i}+\mathbb{R}\langle E\rangle=T \mathbb{R}^{l+1}$, it suffices to show that $\bar{\chi}_{i}^{*}: \operatorname{Ann}(E) \rightarrow \nu^{*}$ is injective, where $\nu^{*}$ is the conormal bundle to $\mathcal{F}$. For, if this is the case, then $\left.D \bar{\chi}_{i}\right|_{\nu}: \nu \rightarrow \operatorname{Ann}(E)^{*}$ is onto and the claim follows. First, it is shown that $\bar{\chi}_{i}^{*}(\operatorname{Ann}(E)) \subset \nu^{*}$; to this end, observe that a transversal $\mathbb{Z}$-projective lattice induces a flat $T \mathcal{F}$-connection on $L \rightarrow N$, which is defined as follows. Let $v \in \Gamma(L)$ and fix $p \in N$; by property (T2), there exists an open neighbourhood $V \subset N$, and a section $j^{1} u \in \Gamma\left(\left.\Sigma\right|_{V}\right)$ with with $u(q) \neq 0$ for all $q \in U$. Then, locally, there exists $f \in C^{\infty}(U)$ with $v=f u$. For $X \in \Gamma\left(\left.T \mathcal{F}\right|_{U}\right)$, define

$$
\nabla_{X} v:=\mathcal{L}_{X}(f) u \text {. }
$$

It can be checked that this is well-defined, that is, indeed, a flat $T \mathcal{F}$-connection on $L \rightarrow N$, and that if $j^{1} u \in \Gamma(\Sigma)$, then $\nabla u=0$. Using this connection, have that there exists a closed, foliated 1-form $\beta_{i}$ such that, for all $r,\left.d g_{r}^{i}\right|_{T \mathcal{F}}=\left.g_{r}^{i} \beta_{i}\right|_{T \mathcal{F}}$. Hence

$$
\left.D \bar{\chi}_{i}\right|_{T \mathcal{F}}=\beta_{i} \otimes E
$$

which implies that $\bar{\chi}_{i}^{*}(\operatorname{Ann}(E)) \subset \nu^{*}$. To show that $\bar{\chi}_{i}^{*}: \operatorname{Ann}(E) \rightarrow \nu^{*}$ is injective, it suffices to show that it is surjective (which is, of course, equivalent by dimension counting). Let $\gamma \in$ $\Gamma\left(\left.\nu^{*}\right|_{U_{i}}\right)$, by property (T2) there exist $f^{1}, \ldots, f^{l+1} \in C^{\infty}\left(U_{i}\right)$ with

$$
\gamma=\left.\left.\sum_{r} f^{r} j^{1} g_{r}^{i} \in \nu^{*} \subset \Sigma^{\mathbb{R}}\right|_{U_{i}} \subset J^{1} L\right|_{U_{i}}
$$

Using the Spencer decomposition, it follows that $\sum_{r} f^{r} g_{r}^{i}=0$ and $\gamma=\sum_{r} f^{r} d g_{r}^{i}$. Therefore, $\bar{\chi}_{i}^{*}\left(\gamma^{\prime}\right)=\gamma$ for

$$
\gamma^{\prime}=\sum_{r} f^{r}(p) \mathrm{d} x_{r} \in \Gamma\left(\left.\operatorname{Ann}(E)\right|_{\bar{\chi}_{i}\left(U_{i}\right)}\right),
$$

and hence surjectivity follows.

Claim C.4. The diagram

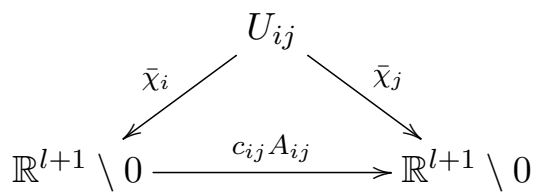

commutes. 
Proof of Claim C.4. This follows by definition of the maps $\bar{\chi}_{i}$.

Using Claims C.3 and C.4, a transversal $\mathbb{Z}$-projective structure can be defined as follows. Set $\chi_{i}: U_{i} \rightarrow \mathbb{R P}^{l}$ to be the composite

$$
\chi_{i}: \quad U_{i} \stackrel{\bar{\chi}_{i}}{\rightarrow} \mathbb{R}^{l+1} \backslash 0 \stackrel{q}{\rightarrow} \mathbb{R P}^{l},
$$

where $q: \mathbb{R}^{l+1} \backslash 0 \rightarrow \mathbb{R} \mathrm{P}^{l}$ is the quotient map. By Claim C.3, $\chi_{i}$ is a submersion, and Claim C.4 implies that $\chi_{j}=\left[A_{i j}\right] \circ \chi_{i}$ on $U_{i j}$. Moreover, since $D q(E)=0$, equation (C.10) implies that $\left.D \chi_{i}\right|_{T \mathcal{F}}=0$, hence the leaves of $\mathcal{F}$ are tangent to the fibres of $\chi_{i}$ on the one hand, and on the other, dimension counting shows that for $p \in U_{i}, T \mathcal{F}_{p}=\operatorname{ker} D_{p} \chi_{i}$.

From structures to lattices. The idea is to pull-back the $\mathbb{Z}$-projective lattice on $\mathbb{R P}^{l}$ constructed in Example 4.15 using the transversal $\mathbb{Z}$-projective structure. Fix a transversal $\mathbb{Z}$-projective structure $\mathcal{A}=\left\{\left(U_{i}, \chi_{i}\right)\right\}$ with cocycle $A_{i j}: U_{i j} \rightarrow \mathrm{GL}(l+1 ; \mathbb{Z})$. On each $i$, set $L_{i}:=\chi_{i}^{*}(O(1))$ and $\Sigma_{i}=\chi_{i}^{*} \Sigma^{l}$, where $\Sigma^{l} \subset J^{1}(O(1))$ is as in Example 4.15. The line bundle $\pi: O(1) \rightarrow \mathbb{R P}^{l}$ is $\mathrm{GL}(l+1 ; \mathbb{Z})$-linearisable in the sense of [24], i.e., there exists an action of $\mathrm{GL}(l+1 ; \mathbb{Z})$ on $O(1)$ which is linear on the fibres of $\pi$ and makes $\pi \mathrm{GL}(l+1 ; \mathbb{Z})$-equivariant. The induced action is the standard GL $(l+1 ; \mathbb{Z})$-action on the vector space $\mathbb{R}\left\langle x_{1}, \ldots, x_{l+1}\right\rangle$ and, thus, it preserves $\Sigma_{l}$. Suppose that $U_{i j} \neq \varnothing$; then

$$
\left.L_{j}\right|_{U_{i j}}=\chi_{j}^{*}(O(1))=\left(\left[A_{i j}\right] \circ \chi_{i}\right)^{*}(O(1))=\chi_{i}^{*}(O(1))=\left.L_{i}\right|_{U_{i j}},
$$

where the third equality uses the fact that $\left[A_{i j}\right]^{*}(O(1))=O(1)$ as $O(1) \rightarrow \mathbb{R P}^{l}$ is $\mathrm{GL}(l+1 ; \mathbb{Z})$ linearised. Therefore, the above construction yields a line bundle $L \rightarrow N$. Similarly, it can be shown that $\left.\Sigma_{j}\right|_{U_{i j}}=\left.\Sigma_{i}\right|_{U_{i j}}$, thus obtaining a $\mathbb{Z}^{l+1}$-bundle $\Sigma \rightarrow N$ whose total space is an embedded submanifold of $J^{1} L$. Therefore property (T1) holds. Property (T2) holds since the submersions $\chi_{i}$ locally define $\mathcal{F}$ (thus showing that $\nu^{*} \otimes L \hookrightarrow \Sigma^{\mathbb{R}}$ ) and because $\left(\Sigma^{l}\right)^{\mathbb{R}}$ surjects onto $O(1)$, thus showing that $\Sigma^{\mathbb{R}} \rightarrow L$ is also onto. Local sections of $\Sigma$ are holonomic by construction, thus showing that property (T3) holds.

\section{Proofs of results from Section 4.3}

Proof of Theorem 4.22. First it is shown that the difference $\Psi_{\sigma}^{*} \theta-\theta_{0} \in \Omega^{1}\left(\operatorname{ker} \rho /\left.\Sigma\right|_{U} ; \pi^{*} L\right)$ is basic, i.e.,

$$
\Psi_{\sigma}^{*} \theta-\theta_{0}=\pi^{*} \beta
$$

for some 1 -form $\beta \in \Omega^{1}(U ; L)$. This is the case if $i_{Z}\left(\Psi_{\sigma}^{*} \theta-\theta_{0}\right)=0$ and $\mathcal{L}_{Z}\left(\Psi_{\sigma}^{*} \theta-\theta_{0}\right)=0$ for any $Z$ tangent to $\operatorname{ker}\left(\pi: \operatorname{ker} \rho /\left.\Sigma\right|_{U} \rightarrow U\right)=: T^{\pi}(\operatorname{ker} \rho / \Sigma)$ (where $\mathcal{L}_{Z} \omega$ is defined as in equation (C.1)). In order to prove this, identify (canonically) $T_{z}^{\pi}\left(\operatorname{ker}_{p} / \Sigma_{p}\right)$ with $\operatorname{ker} \rho_{\pi(z)}$ via the isomorphism $T^{\pi}(\operatorname{ker} \rho / \Sigma) \simeq \pi^{*} \operatorname{ker} \rho$. As $\Psi_{\sigma}$ comes from the action of equation (4.3), a straightforward computation shows that for $\left(u^{\prime}, \eta^{\prime}\right),(u, \eta) \in \Gamma(\operatorname{ker} \rho)$,

$$
\Psi_{\sigma}\left(\left(u^{\prime}, \eta^{\prime}\right)+t(u, \eta)\right)=\varphi_{(u, \eta)}^{t}\left(\Psi_{\sigma}\left(u^{\prime}, \eta^{\prime}\right)\right)
$$

for any $t \in \mathbb{R}$, where $\varphi_{(u, \eta)}^{t}: M \rightarrow M$ is the flow of $\psi\left(\phi^{*}(u, \eta)\right)$ and the Spencer decomposition has been used. Equivalently,

$$
\Psi_{\sigma} \circ \varphi_{(u, v)}^{t}=\varphi_{(u, v)}^{t} \circ \Psi_{\sigma},
$$

where on the left hand side $\varphi_{(u, v)}^{t}: \operatorname{ker} \rho / \Sigma \rightarrow \operatorname{ker} \rho / \Sigma$ stands for the flow $z \mapsto z+t(u, \eta)(\pi(z))$ of the vertical vector field $\pi^{*}(u, \eta)$. Differentiating equation (C.12), obtain that

$$
D_{z} \Psi_{\sigma}\left(\pi^{*}(u, \eta)\right)=\psi\left(\phi^{*} u, \phi^{*} \eta\right)_{\Psi_{\sigma}(z)} .
$$


Let $Z=\pi^{*}(u, \eta)$; by definition of $\psi$ and the fact that for contact manifold $\rho_{M}(\alpha, \gamma)=R_{\alpha}+c^{\sharp}(\gamma)$, for $(\alpha, \gamma) \in \Gamma\left(L_{M}\right) \oplus \Omega^{1}\left(M ; L_{M}\right)$, for $R_{\alpha}$ the Reeb vector field of $\alpha$ (cf. Example 2.5), and $c$ the curvature map of equation (2.1), the above yields

$$
i_{Z} \Psi_{\sigma}^{*} \theta=\theta\left(\psi\left(\phi^{*} u, \phi^{*} \eta\right)\right)=\bar{\theta}\left(R_{F \phi^{*} u}+c\left(\left.F \phi^{*} \eta\right|_{H}\right)\right)=u .
$$

On the other hand, $\theta_{0}$ restricted to $\pi^{*} \operatorname{ker} \rho$ is equal to the projection $\operatorname{pr}: \operatorname{ker} \rho \rightarrow L$ which implies that $i_{Z} \theta_{0}=\theta_{0}(u, \eta)=u$. Thus $i_{Z}\left(\Psi_{\sigma}^{*} \theta-\theta_{0}\right)=0$ follows. To compute the Lie derivative along $Z$, observe that

$$
\mathcal{L}_{Z}\left(\Psi_{\sigma}^{*} \theta\right)=\left.\frac{d}{d t}\left(\varphi_{(u, \eta)}^{t}\right)^{*}\left(\Psi_{\sigma}^{*} \theta\right)\right|_{t=0}=\left.\frac{d}{d t} \Psi_{\sigma}^{*} \circ\left(\varphi_{(u, \eta)}^{t}\right)^{*} \theta\right|_{t=0},
$$

where the second equality uses equation (C.12). By equation (C.3), have that

$$
\left.\frac{d}{d t}\left(\varphi_{(u, \eta)}^{t}\right)^{*} \theta\right|_{t=0}=\phi^{*} \eta
$$

which implies that $\mathcal{L}_{Z} \Psi_{\sigma}^{*} \theta=\pi^{*} \eta$. On the other hand,

$$
\mathcal{L}_{Z} \theta_{0}=\left.\frac{d}{d t}\left(\varphi_{(u, \eta)}^{t}\right)^{*} \theta_{0}\right|_{t=0}=\left.\frac{d}{d t}\left(\mathrm{id}^{*}+t \pi^{*} \circ(u, \eta)^{*}\right) \theta_{0}\right|_{t=0}=\left.\frac{d}{d t}\left(\theta_{0}+t \pi^{*} \eta\right)\right|_{t=0}=\pi^{*} \eta,
$$

where the third equality uses that for a section $(u, \eta): P \rightarrow J^{1} L,(u, \eta)^{*} \theta_{\text {can }}=\eta$. With this, $\mathcal{L}_{Z}\left(\Psi_{\sigma}^{*} \theta-\theta_{0}\right)=0$. As vector fields of the form $\pi^{*}(u, v)$ generate $\pi^{*} \operatorname{ker} \rho \simeq T^{\pi}(\operatorname{ker} \rho / \Sigma)$ as a $C^{\infty}(\operatorname{ker} \rho / \Sigma)$-module, this implies that equation (C.11) holds.

To show that $\beta=\sigma^{*} \theta$, consider the section $z: P \rightarrow \operatorname{ker} \rho / \Sigma, p \mapsto\left[\Sigma_{p}\right]=0$ of $\pi: \operatorname{ker} / \Sigma \rightarrow P$. Then $z^{*} \operatorname{pr}^{*} \beta=\beta, z^{*} \Psi_{\sigma}^{*} \theta=\sigma^{*} \theta$ as $\Psi_{\sigma} \circ z=\sigma$, and $z^{*} \theta_{0}=0$ as for any $s \in \Gamma(\Sigma), s^{*} \theta_{\text {can }}=0$ (see Note 4.21). Therefore, $\beta=\sigma^{*} \theta$.

It remains to show that $\mathrm{d}_{\mathcal{F}}\left(\sigma^{*} \theta\right)=\omega_{\mathcal{F}}$. As $\Gamma(\mathcal{F})$ is generated by elements of the form $\rho\left(j^{1} v\right), v \in \Gamma(L)$, it suffices to check the statements for vector fields of this form. By definition,

$$
\begin{aligned}
\mathrm{d}_{\mathcal{F}}\left(\sigma^{*} \theta\right)\left(\rho\left(j^{1} u\right), \rho\left(j^{1} v\right)\right)= & -\sigma^{*} \theta\left(\left[\rho\left(j^{1} u\right), \rho\left(j^{1} v\right)\right]\right) \\
& +\nabla_{\rho\left(j^{1} u\right)}\left(\sigma^{*} \theta\left(\rho\left(j^{1} v\right)\right)\right)-\nabla_{\rho\left(j^{1} v\right)}\left(\sigma^{*} \theta\left(\rho\left(j^{1} u\right)\right)\right) .
\end{aligned}
$$

First, consider $\nabla_{\rho\left(j^{1} u\right)}\left(\sigma^{*} \theta\left(\rho\left(j^{1} v\right)\right)\right)=\nabla_{\rho\left(j^{1} u\right)}\left(\theta\left(D \sigma\left(\rho\left(j^{1} v\right)\right)\right)\right)$; since $D \phi \circ D \sigma=$ id and $\phi$ is a Jacobi map, the diagram of Note 2.13 commutes. Thus

$$
D \sigma\left(\rho\left(j^{1} v\right)\right)=\rho_{M}\left(j^{1}\left(\phi^{*} v\right)\right)+X_{v}
$$

where $X_{v} \in \Gamma\left(\left.\operatorname{ker} D \phi\right|_{\sigma(U)}\right)$ (and similarly for $u$ ).

As $\phi$ satisfies property (I), ker $D \phi=\rho_{M}\left(\phi^{*} \operatorname{ker} \rho\right)$, hence there exist local sections $(w, \eta),(z, \zeta)$ $\in \Gamma(\operatorname{ker} \rho)$ with

$$
X_{u}=\rho_{M}\left(\phi^{*} w, \phi^{*} \eta\right) \quad \text { and } \quad X_{v}=\rho_{M}\left(\phi^{*} z, \phi^{*} \zeta\right) .
$$

Computing $\rho_{M}$ for a contact manifold,

$$
\theta\left(D \sigma\left(\rho\left(j^{1} v\right)\right)\right)=\theta\left(R_{\phi^{*} v}+R_{\phi^{*} z}+c^{\sharp}\left(\left.\phi^{*} \zeta\right|_{H}\right)\right)=\phi^{*}(v+z),
$$

and, similarly, $\theta\left(D \sigma\left(\rho\left(j^{1} u\right)\right)\right)=\phi^{*}(u+w)$. Therefore, by definition of $\nabla$,

$$
\nabla_{\rho\left(j^{1} u\right)}\left(\sigma^{*} \theta\left(\rho\left(j^{1} v\right)\right)\right)-\nabla_{\rho\left(j^{1} v\right)}\left(\sigma^{*} \theta\left(\rho\left(j^{1} u\right)\right)\right)=\{u, v+z\}-\{v, u+w\} .
$$

On the other hand,

$$
\left(\sigma^{*} \theta\right)\left(\left[\rho\left(j^{1} u\right), \rho\left(j^{1} v\right)\right]\right)=\sigma^{*}\left(\theta\left(D \sigma\left(\left[\rho\left(j^{1} u\right), \rho\left(j^{1} v\right)\right]\right)\right)\right)
$$




$$
\begin{aligned}
& =\sigma^{*}\left(\theta\left(\left(\left[D \sigma\left(\rho\left(j^{1} u\right)\right), D \sigma\left(\rho\left(j^{1} v\right)\right)\right]\right)\right)\right) \\
& =\sigma^{*}\left(\theta\left(\left[R_{\phi^{*}(u+w)}+c^{\sharp}\left(\left.\phi^{*} \eta\right|_{H}\right), R_{\phi^{*}(v+z)}+c^{\sharp}\left(\left.\phi^{*} \zeta\right|_{H}\right)\right]\right)\right) \\
& =\sigma^{*}\left(\theta\left(R_{\left\{\phi^{*}(u+w), \phi^{*}(v+z)\right\}_{M}}+\left[c^{\sharp}\left(\left.\phi^{*} \eta\right|_{H}\right), c^{\sharp}\left(\left.\phi^{*} \zeta\right|_{H}\right)\right]\right)\right) \\
& =\sigma^{*}\left(\phi^{*}\left(\{u+w, v+z\}_{M}\right)-\phi^{*} \eta\left(\rho_{M}\left(\phi^{*} \zeta\right)\right)\right) \\
& =\{u+w, v+z\}-\eta\left(D \phi\left(\rho_{M}\left(\phi^{*} \zeta\right)\right)\right)=\{u+w, v+z\}-\eta(\rho(\zeta)),
\end{aligned}
$$

where the fourth equality follows from the defining property of Reeb vector fields (cf. Example 2.5), the fifth by definition of Reeb vector fields and the curvature map of equation (2.1), and the last from the fact that $\phi$ is a Jacobi map. Observe that since $(w, \eta),(z, \zeta) \in \Gamma(\operatorname{ker} \rho)$,

$$
\{w, z\}-\eta(\rho(\zeta))=\{w, z\}+\eta\left(\rho\left(j^{1} z\right)\right)=\omega(\rho(w, \eta), \rho(z, \zeta))=0,
$$

where the general definition of $\omega_{\mathcal{F}}$ is used (cf. Note 3.8). Thus equation (C.15) yields that

$$
\left(\sigma^{*} \theta\right)\left(\left[\rho\left(j^{1} u\right), \rho\left(j^{1} v\right)\right]\right)=\{u, v\}+\{u, z\}+\{w, v\} .
$$

Using this identity together with equation (C.14) in equation (C.13) yields the required result.

\section{Acknowledgements}

We would like to thank two anonymous referees for the suggestions that helped improve significantly the content and its presentation. Furthermore, we would like to thank Camilo Arias Abad for interesting conversations. M.A.S. would like to thank IMPA, CRM and MPIM Bonn for hospitality at various stages of the project. M.A.S. was partly supported by the DevMath programme of the Centre de Recerca Matemàtica and by the Max Planck Institute for Mathematics in Bonn. D.S. was partly supported by ERC starting grant 279729, by the NWO Veni grant 639.031.345 and by CNPq.

\section{References}

[1] Banyaga A., Molino P., Géométrie des formes de contact complètement intégrables de type toriques, in Séminaire Gaston Darboux de Géométrie et Topologie Différentielle, 1991-1992 (Montpellier), Univ. Montpellier II, Montpellier, 1993, 1-25.

[2] Boothby W.M., Wang H.C., On contact manifolds, Ann. of Math. 68 (1958), 721-734.

[3] Coste A., Dazord P., Weinstein A., Groupoïdes symplectiques, in Publications du Département de Mathématiques. Nouvelle Série. A, Vol. 2, Publ. Dép. Math. Nouvelle Sér. A, Vol. 87, Univ. Claude-Bernard, Lyon, 1987, i-ii, 1-62.

[4] Crainic M., Fernandes R.L., Integrability of Poisson brackets, J. Differential Geom. 66 (2004), 71-137, math.DG/0210152.

[5] Crainic M., Fernandes R.L., Martínez Torres D., Poisson manifolds of compact types (PMCT 1), J. Reine Angew. Math., to appear, arXiv:1510.07108.

[6] Crainic M., Fernandes R.L., Martínez Torres D., Poisson manifolds of compact types (PMCT 2), arXiv:1603.00064.

[7] Crainic M., Salazar M.A., Jacobi structures and Spencer operators, J. Math. Pures Appl. 103 (2015), 504521, arXiv:1309.6156.

[8] Crainic M., Salazar M.A., Struchiner I., Multiplicative forms and Spencer operators, Math. Z. 279 (2015), 939-979, arXiv:1210.2277.

[9] Crainic M., Zhu C., Integrability of Jacobi and Poisson structures, Ann. Inst. Fourier (Grenoble) 57 (2007), 1181-1216, math.DG/0403268.

[10] Dazord P., Sur l'intégration des algèbres de Lie locales et la préquantification, Bull. Sci. Math. 121 (1997), 423-462. 
[11] Dazord P., Delzant T., Le problème général des variables actions-angles, J. Differential Geom. 26 (1987), 223-251.

[12] Dazord P., Lichnerowicz A., Marle C.-M., Structure locale des variétés de Jacobi, J. Math. Pures Appl. 70 (1991), 101-152.

[13] de León M., López B., Marrero J.C., Padrón E., On the computation of the Lichnerowicz-Jacobi cohomology, J. Geom. Phys. 44 (2003), 507-522.

[14] Duistermaat J.J., Kolk J.A.C., Lie groups, Universitext, Springer-Verlag, Berlin, 2000.

[15] El Kacimi-Alaoui A., Sur la cohomologie feuilletée, Compositio Math. 49 (1983), 195-215.

[16] Geiges H., An introduction to contact topology, Cambridge Studies in Advanced Mathematics, Vol. 109, Cambridge University Press, Cambridge, 2008.

[17] Gross M., Siebert B., Mirror symmetry via logarithmic degeneration data. I, J. Differential Geom. 72 (2006), 169-338, math.AG/0309070.

[18] Guedira F., Lichnerowicz A., Géométrie des algèbres de Lie locales de Kirillov, J. Math. Pures Appl. 63 (1984), 407-484.

[19] Guillemin V., Sjamaar R., Convexity properties of Hamiltonian group actions, CRM Monograph Series, Vol. 26, Amer. Math. Soc., Providence, RI, 2005.

[20] Guillemin V., Sternberg S., A normal form for the moment map, in Differential Geometric Methods in Mathematical Physics (Jerusalem, 1982), Math. Phys. Stud., Vol. 6, Reidel, Dordrecht, 1984, 161-175.

[21] Jovanović B., Noncommutative integrability and action-angle variables in contact geometry, J. Symplectic Geom. 10 (2012), 535-561, arXiv:1103.3611.

[22] Kerbrat Y., Souici-Benhammadi Z., Variétés de Jacobi et groupoïdes de contact, C. R. Acad. Sci. Paris Sér. I Math. 317 (1993), 81-86.

[23] Kirillov A.A., Local Lie algebras, Russ. Math. Surv. 31 (1976), no. 4, 55-76.

[24] Knop F., Kraft H., Luna D., Vust T., Local properties of algebraic group actions, in Algebraische Transformationsgruppen und Invariantentheorie, DMV Sem., Vol. 13, Birkhäuser, Basel, 1989, 63-75.

[25] Lerman E., Contact toric manifolds, J. Symplectic Geom. 1 (2003), 785-828, math.SG/0107201.

[26] Lichnerowicz A., Les variétés de Jacobi et leurs algèbres de Lie associées, J. Math. Pures Appl. 57 (1978), 453-488.

[27] Marle C.-M., Modèle d'action hamiltonienne d'un groupe de Lie sur une variété symplectique, Rend. Sem. Mat. Univ. Politec. Torino 43 (1985), 227-251.

[28] McDuff D., Salamon D., Introduction to symplectic topology, 2nd ed., Oxford Mathematical Monographs, The Clarendon Press, Oxford University Press, New York, 1998.

[29] Ortega J.-P., Ratiu T.S., A symplectic slice theorem, Lett. Math. Phys. 59 (2002), 81-93, math.SG/0110084.

[30] Sjamaar R., Lerman E., Stratified symplectic spaces and reduction, Ann. of Math. 134 (1991), 375-422.

[31] Weinstein A., Symplectic groupoids and Poisson manifolds, Bull. Amer. Math. Soc. (N.S.) 16 (1987), 101104.

[32] Wolbert S., Symplectic toric stratified spaces with isolated singularities, arXiv:1510.05740.

[33] Zambon M., Zhu C., Contact reduction and groupoid actions, Trans. Amer. Math. Soc. 358 (2006), 13651401, math.DG/0405047. 